\title{
Investigation of Shape Memory Alloy Wire for Closed Loop Angular Position Control of a Shaft under Random Excitation
}

\author{
by \\ Brian Lynch \\ Thesis submitted to the \\ Faculty of Graduate Studies and Research \\ in partial fulfillment of the degree \\ Masters of Applied Sciences in Aerospace Engineering \\ Department of Mechanical \& Aerospace Engineering \\ Carleton University \\ Ottawa, Canada
}

September 2007

(C) Brian Lynch, 2007 


$\begin{array}{ll}\begin{array}{l}\text { Library and } \\ \text { Archives Canada }\end{array} & \begin{array}{l}\text { Bibliothèque et } \\ \text { Archives Canada }\end{array} \\ \begin{array}{l}\text { Published Heritage } \\ \text { Branch }\end{array} & \begin{array}{l}\text { Direction du } \\ \text { Patrimoine de l'édition }\end{array} \\ \begin{array}{l}\text { 395 Wellington Street } \\ \text { Ottawa ON K1A ON4 }\end{array} & \begin{array}{l}\text { 395, rue Wellington } \\ \text { Ottawa ON K1A ON4 } \\ \text { Canada }\end{array}\end{array}$

Your file Votre référence ISBN: 978-0-494-33660-1 Our file Notre référence ISBN: 978-0-494-33660-1

NOTICE:

The author has granted a nonexclusive license allowing Library and Archives Canada to reproduce, publish, archive, preserve, conserve, communicate to the public by telecommunication or on the Internet, loan, distribute and sell theses worldwide, for commercial or noncommercial purposes, in microform, paper, electronic and/or any other formats.

The author retains copyright ownership and moral rights in this thesis. Neither the thesis nor substantial extracts from it may be printed or otherwise reproduced without the author's permission.
AVIS:

L'auteur a accordé une licence non exclusive permettant à la Bibliothèque et Archives Canada de reproduire, publier, archiver, sauvegarder, conserver, transmettre au public par télécommunication ou par l'Internet, prêter, distribuer et vendre des thèses partout dans le monde, à des fins commerciales ou autres, sur support microforme, papier, électronique et/ou autres formats.

L'auteur conserve la propriété du droit d'auteur et des droits moraux qui protège cette thèse. $\mathrm{Ni}$ la thèse ni des extraits substantiels de celle-ci ne doivent être imprimés ou autrement reproduits sans son autorisation.
In compliance with the Canadian

Privacy Act some supporting forms may have been removed from this thesis.

While these forms may be included in the document page count, their removal does not represent any loss of content from the thesis.
Conformément à la loi canadienne sur la protection de la vie privée, quelques formulaires secondaires ont été enlevés de cette thèse.

Bien que ces formulaires aient inclus dans la pagination, il n'y aura aucun contenu manquant.

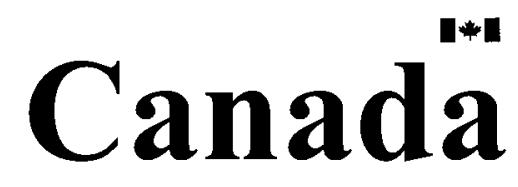




\section{Abstract}

Shape memory alloys (SMA) exhibit many positive characteristics for application in smart structures. Control of SMA actuators has typically been limited by their nonlinear behaviour. Actuators using SMA are restricted to low frequency applications due to the necessary cooling times. Nevertheless, SMA actuators are applied for active control systems within experimental aerospace vehicles. This thesis is an investigation of SMA actuator wires for control of a shaft angle. A review of SMA actuator applications and actively controlled helicopter rotor blades is presented. Experiments performed to determine the characteristics of the Flexinol SMA wire are then discussed. Control of the shaft is demonstrated using a single actuator wire and bias load, and two antagonistic wires. Casual control algorithms developed to successfully follow a target angle and reject random disturbances are presented. A comparison is made between experimental data and mathematical models. Finally, the use of SMA actuator wires for control of an active helicopter blade tip is studied for feasibility. 


\section{Contents}

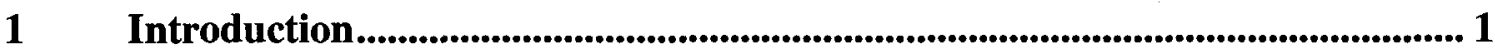

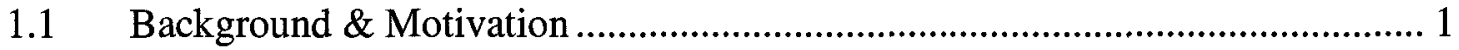

1.2 Shape Memory Alloy Characteristics ............................................................ 3

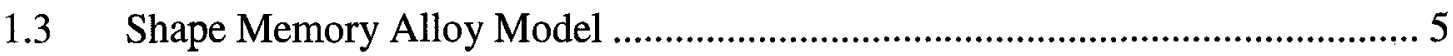

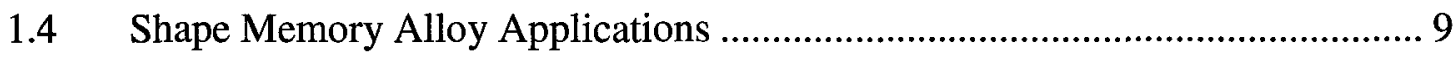

1.4.1 SMA Actuated Microvalves ................................................................... 9

1.4.2 Miniature SMA Actuated Grippers...................................................... 11

1.4.3 SMA Actuator for Tactile Information Display................................... 13

1.4.4 Miniature Rotary SMA Latching Device............................................... 14

1.4.5 Modular Machine using Rotary SMA Actuators ................................... 15

1.5 Actively Controlled Rotor Blades.................................................................. 16

1.5.1 Smart Material-Actuated Rotor Technology (SMART) .......................... 16

1.5.2 Helicopter Blade with Integrated Smart Morphing Actuator ................... 18

1.5.3 Smart Hybrid Active Rotor Control System (SHARCS).......................... 20

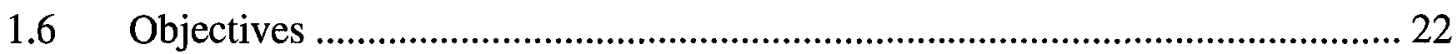

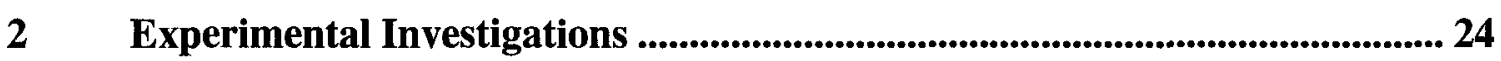

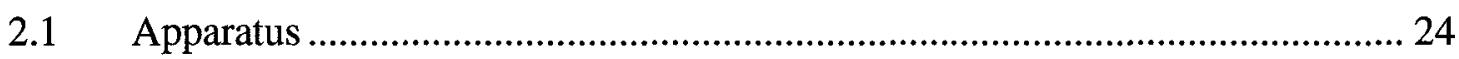

2.1.1 Flexinol Shape Memory Alloy Actuator Wire...................................... 25

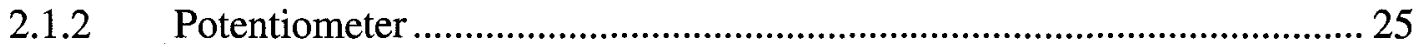

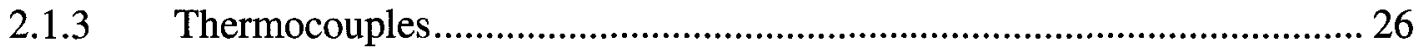

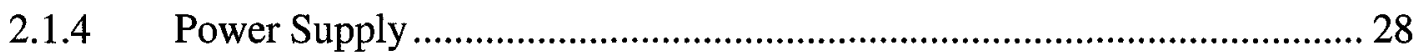

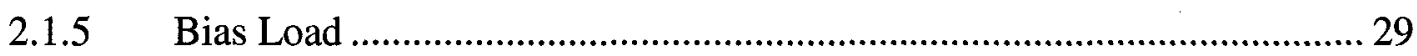

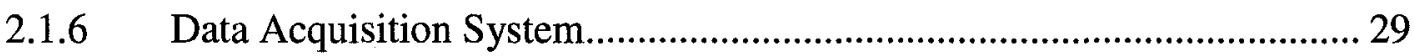

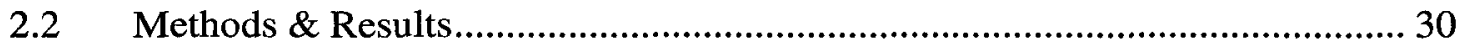

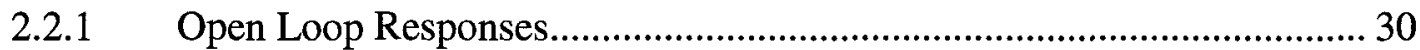

2.2.2 Material Characterization................................................................... 33

2.2.3 Single Channel Control Strategies ...................................................... 37

2.2.4 Dual Channel Control Strategies ....................................................... 45 
3 Mathematical Models 58

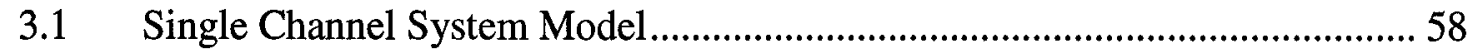

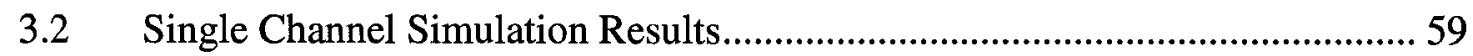

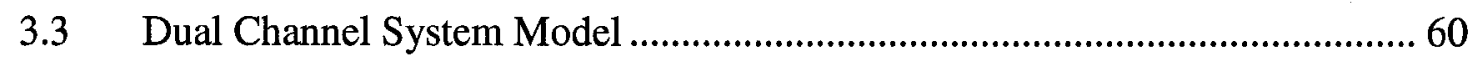

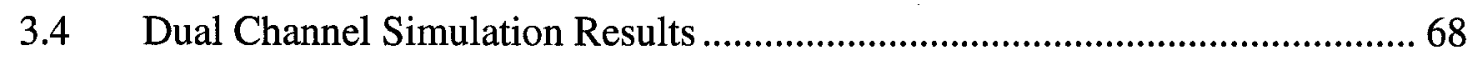

4 Applications to SHARCS Project ......................................................................... 70

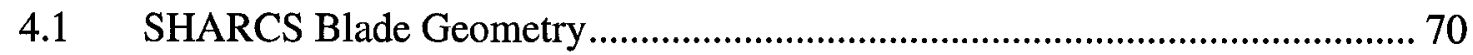

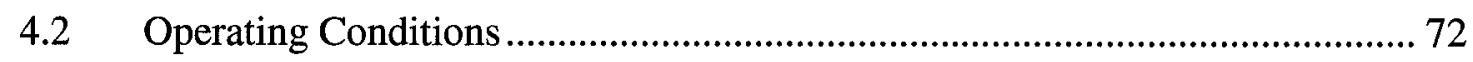

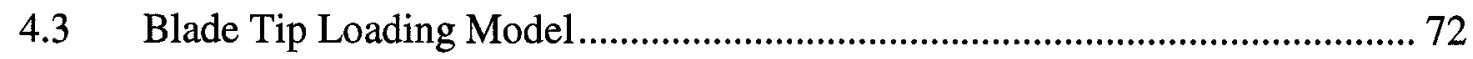

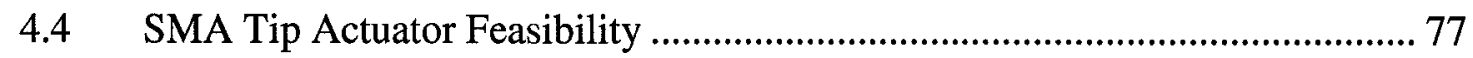

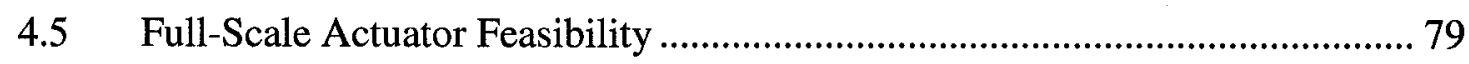

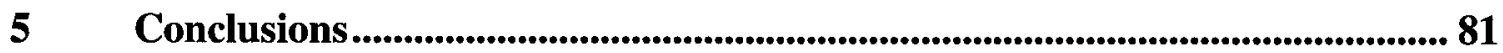

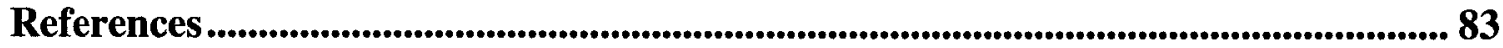




\section{List of Tables}

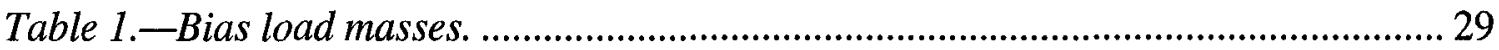

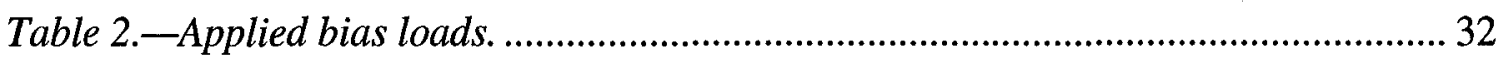

Table 3.-Flexinol material properties assumed from literature ..................................... 34

Table 4.-Flexinol material properties determined from experiment. ............................ 36

Table 5.-Applied current values for control algorithm $I$. ......................................... 38

Table 6.-Applied current logic for control algorithm II. .......................................... 40

Table 7.-Modified applied current logic for control algorithm II................................. 41

Table 8.-Conditions for casual control algorithm III. ................................................... 41

Table 9.-Current increments and regional gains for control algorithm IV.................... 43

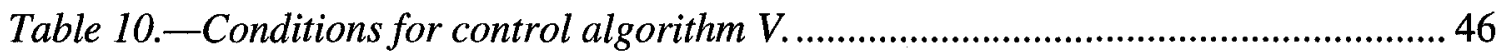

Table 11.-Modified conditions and current values for control algorithm $V$. ................. 47

Table 12.-Conditions for control algorithm VI. ........................................................... 48

Table 13.-Modified conditions for control algorithm VII........................................... 50

Table 14.-Experimentally determined values for coefficients in equation 15............... 54

Table 15._Gains determined through Ziegler-Nichols tuning method I. ........................ 55

Table 16.-Gains determined through Ziegler-Nichols tuning method II. ....................... 56

Table 17. - Single channel model simulation parameters. ................................................. 59

Table 18._Dual channel model simulation parameters. ..................................................68

Table 19.-Parameters assumed from a full scale Bo-105 rotor blade. ......................... 79 


\section{List of Figures}

Figure 1.-NASA/Boeing SMA actuated jet engine chevron. ............................................. 2

Figure 2.—SMA stress-strain-temperature curve........................................................ 4

Figure 3.—CWRU Biorobotics Autonomous Hybrid Robot. ........................................... 9

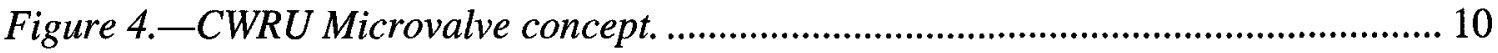

Figure 5.-KUL implantable drug delivery system microvalve design............................ 11

Figure 6.-University of Tokyo SMA actuated clean gripper. ........................................ 12

Figure 7.-Institute of Microtechnology SMA actuated gripper..................................... 12

Figure 8.-Laboratoire de Robotique de Paris SMA actuator concept ............................ 13

Figure 9.-Laboratoire de Robotique de Paris display grid concept. ............................. 14

Figure 10.-Miniature SMA rotary latching device ...................................................... 15

Figure 11.-DSYSD modular machine concept ......................................................... 15

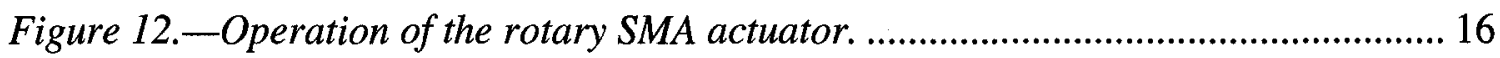

Figure 13.-Boeing SMART rotor blade concept ............................................................ 17

Figure 14._SMART rotor blade actuation mechanisms. ................................................ 18

Figure 15._IARC actively controlled rotor blade tip concept ........................................ 19

Figure 16.-SHARCS rotor blade with ACT, ACF, and APL subsystems........................ 20

Figure 17.-Conventional pitch link and Active Pitch Link concept............................... 21

Figure 18._Flow chart representation of experimental setup........................................ 24

Figure 19.-Thermocouple signal conditioner calibration data.................................... 27

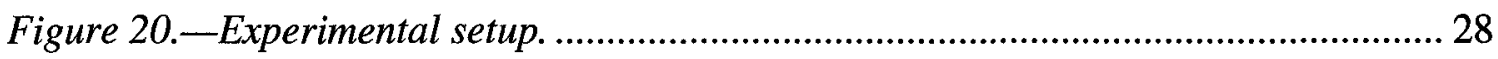

Figure 21.-SMA actuator wire cycling data .................................................................. 31

Figure 22.-Open loop responses to varying bias loads................................................. 32

Figure 23.-Transformation temperature extrapolation deviation. ................................ 35

Figure 24.-Austenitic transformation temperatures. ...................................................... 36

Figure 25.-Martensitic transformation temperatures................................................... 37

Figure 26.-.Shaft response using control algorithm I. ................................................. 39

Figure 27.-Shaft response using control algorithm III................................................. 42

Figure 28. -Shaft response using casual control algorithm IV. .................................... 45 
Figure 29.-Shaft response using control algorithm $V$.............................................. 48

Figure 30.-Shaft response using modified control algorithm VI................................. 49

Figure 31.-Shaft response using control algorithm VII................................................ 51

Figure 32.-Shaft response using control algorithm VIII. ........................................... 52

Figure 33.-Visualization of current output for control algorithm IX.......................... 53

Figure 34.-Shaft response using control algorithm VIII. .............................................. 54

Figure 35.- Single channel model simulation results. ..................................................60

Figure 36. - Flow chart of Newton-Raphson solution method ...................................... 67

Figure 37._Dual channel model simulation results. ....................................................69

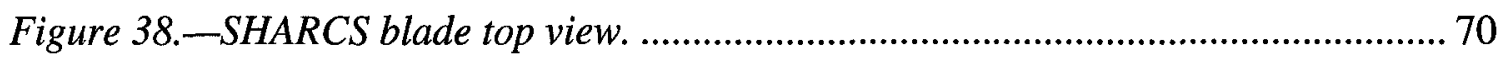

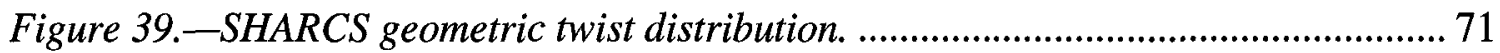

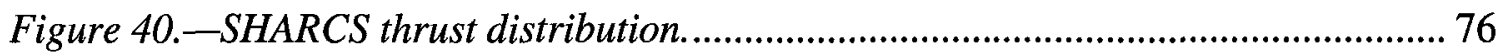

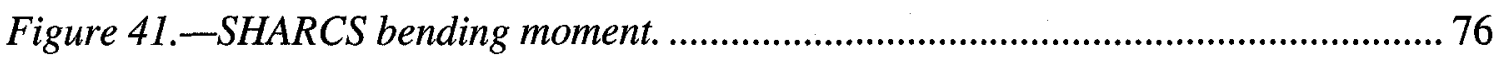

Figure 42.-SHARCS net hinge moment for various anhedral angle settings. ............... 77

Figure 43._Possible configuration for SHARCS tip hinge.............................................. 78

Figure 44.-Tip hinge moment for full scale SHARCS.................................................. 80 


\section{Nomenclature}

a Slope of lift coefficient curve.

A Rotor disk area.

$A_{f} \quad$ Austenite finish transformation temperature.

$A_{s} \quad$ Austenite start transformation temperature.

c Blade chord length.

$C_{A} \quad$ Austenite stress influence coefficient.

$C_{M} \quad$ Martensite stress influence coefficient.

$C_{T} \quad$ Thrust coefficient.

E Young's modulus.

$E_{A} \quad$ Austenite Young's modulus of elasticity.

$E_{M} \quad$ Martensite Young's modulus of elasticity.

F Prandtl tip loss factor.

$h \quad$ Tip hinge radial location.

$I_{0} \quad$ Pre-amplified control current.

$I_{\text {off }} \quad$ Offset control current.

$I_{\text {out }} \quad$ Output control current.

$I_{\text {ref }} \quad$ Reference control current.

$K_{p} \quad$ PID proportional gain.

$K_{u} \quad$ Ziegler-Nichols ultimate gain.

$L \quad$ Ziegler-Nichols dead time; SMA wire length.

$M_{A} \quad$ Aerodynamic tip hinge moment.

$M_{C} \quad$ Centrifugal tip hinge moment.

$M_{f} \quad$ Martensite finish transformation temperature.

$M_{s} \quad$ Martensite start transformation temperature.

$M_{T} \quad$ Total tip hinge moment.

$N \quad$ Number of blades.

$P_{c r} \quad$ Ziegler-Nichols critical period.

$r \quad$ Shaft radius; normalized radial location.

viii 
$R \quad$ Rotor blade radius.

$T$ Temperature; Ziegler-Nichols time constant; thrust.

$T_{d} \quad$ PID derivative time constant.

$T_{i} \quad$ PID integral time constant.

$x \quad$ Radial location variable.

$z \quad$ Radial location variable.

$\alpha \quad$ Signal conditioner voltage gain; control coefficient; coefficient of thermal expansion; Newton-Raphson solution variable.

$\alpha_{A} \quad$ Austenite thermal expansion coefficient.

$\alpha_{M} \quad$ Martensite thermal expansion coefficient.

$\beta \quad$ Signal conditioner voltage offset; control coefficient; tip anhedral angle; NewtonRaphson solution variable.

$\gamma \quad$ Control current gain; control coefficient; Newton-Raphson solution variable.

$\delta \quad$ Control coefficient.

$\delta$ Control current increment.

$\Delta \quad$ Shaft angular error.

$\varepsilon \quad$ Strain; compound error signal.

$\varepsilon_{L} \quad$ Maximum residual strain.

$\eta \quad$ Newton-Raphson convergence residual.

$\lambda \quad$ Inflow ratio.

$\mu \quad$ Blade mass per unit area.

$\theta \quad$ Shaft angle; blade geometric twist.

$\Theta \quad$ Thermal expansion modulus.

$\rho \quad$ Air density.

$\sigma \quad$ Stress; rotor solidity.

$\tau \quad$ Shaft target angle.

$\omega \quad$ Shaft angular speed.

$\Omega$ Transformation tensor; rotor rotational velocity. 
$\Psi \quad$ Directional control factor.

$\xi \quad$ Martensite fraction.

$\xi_{0} \quad$ Initial martensite fraction. 


\section{Introduction}

\subsection{Background \& Motivation}

Smart materials, such as piezoelectric ceramics and shape memory alloys, have become a popular alternative to conventional actuation methods. Hydraulic, pneumatic, or electromechanical actuators typically require a complex structure of support mechanisms. For example, a hydraulic actuator on a commercial passenger jet requires filters, pumps, reservoirs, and an elaborate network of conduit lines in order to provide and maintain the desired actuation capabilities. A similar system using smart materials could be almost entirely localized with very little support structure required-decreasing the effective mass of the actuator. The most commonly used smart materials are piezoelectric ceramics and shape memory alloys.

Piezoelectric materials demonstrate a coupling between applied stress and electric polarization. The piezoelectric effect results in a voltage developed across the material when subjected to an applied stress. Furthermore, an applied voltage leads to a stress or strain depending upon the boundary conditions. Piezoelectric materials offer the ability to precisely position an object to within nanometre resolution and have a wide range of operating frequencies. These properties make them ideal in applications where very accurate positioning is required or where vibration attenuation is a primary goal.

Shape memory alloys exhibit a similar coupled effect between temperature and mechanical deformation. The phenomenal behaviour of shape memory alloys can be separated into two categories: shape memory effect and superelasticity. The shape memory effect is demonstrated when the alloy appears to be permanently deformed but 
the application of heat restores the shape to a previous geometry. Superelasticity, while dependent upon the same physical processes, is demonstrated by the alloy's ability to recover a very high deformation without the application of heat. The difference between the two processes is dependent upon the surrounding temperature and applied stress. Both superelasticity and the shape memory effect may be exhibited by the same material, yet under different conditions. The shape memory effect is favoured over superelasticity for actuator applications, since it is desirable to actively control the strain recovery.

Research on the use of shape memory alloys for actuation has been primarily focused on on/off control due to the highly non-linear relationship between stress, strain and temperature. Actuators employing shape memory alloys cannot typically reach intermediate positions within the stroke. Active cooling is also difficult to implement in most shape memory alloy based actuators, causing a large response time for the actuator to return to its original configuration. Despite these problems, research and development

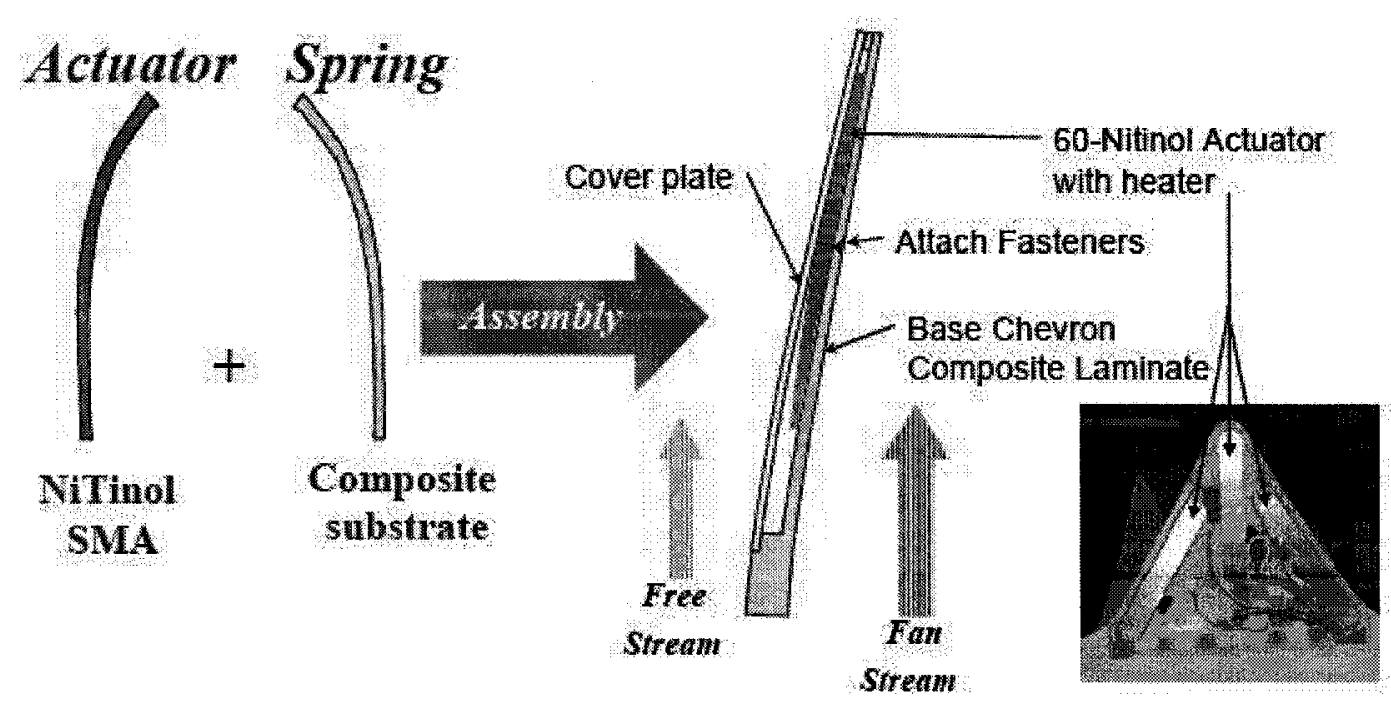

Figure 1.-NASA/Boeing SMA actuated jet engine chevron [1]. 
of actuation systems using shape memory alloy continues to demonstrate positive results. Shape memory alloy wires or strips may be integrated into composite structures to achieve variable geometry capabilities. Research by NASA and Boeing has led to a shape memory alloy actuated active jet engine chevron shown in Figure 1 [1] [2]. The static chevrons-arranged around the exhaust nozzle of the engine-may be actively protruded into the exhaust flow region in order to reduce engine noise during take-off and landing.

\subsection{Shape Memory Alloy Characteristics}

Shape memory alloys behave similar to conventional metals but undergo phenomenal crystallization processes when subject to specific levels of stress and temperature. As previously mentioned, shape memory alloys can recover great amounts of strain through the shape memory effect or superelasticity. The material consists of two phases: martensite and austenite. It should be noted that there exists two types of martensite (stress-induced and temperature-induced), however, for simplicity they will not be dissociated. Martensite is dominant at both high stress and low temperatures, whereas austenite is dominant at low stress and high temperatures. The phenomena of superelasticity and shape memory effect are both attributed to the transformation of the material between martensite and austenite. Although the material appears to be plastic there is simply a transformation of the internal structure resulting in an apparent permanent deformation. When recovering a stored geometry, the material transforms from martensite to austenite whereby the previous crystal structure is re-established. 


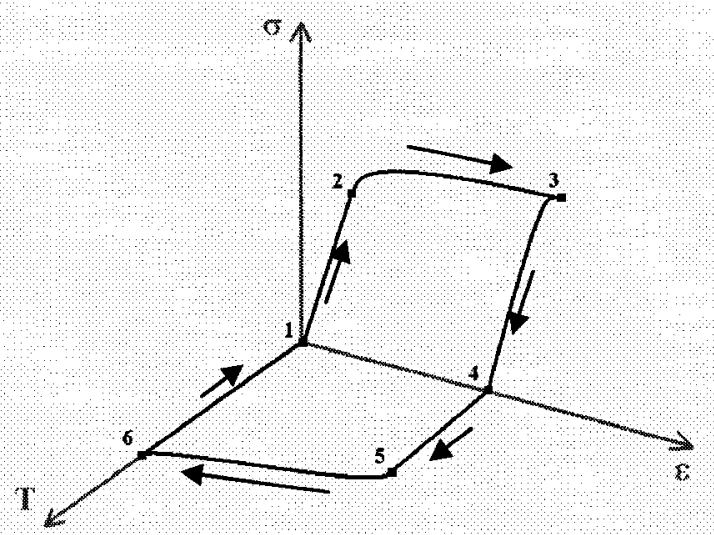

(a)

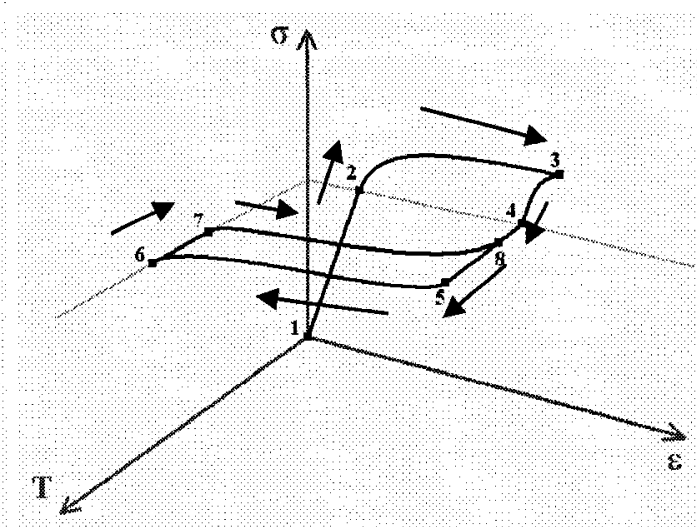

(b)

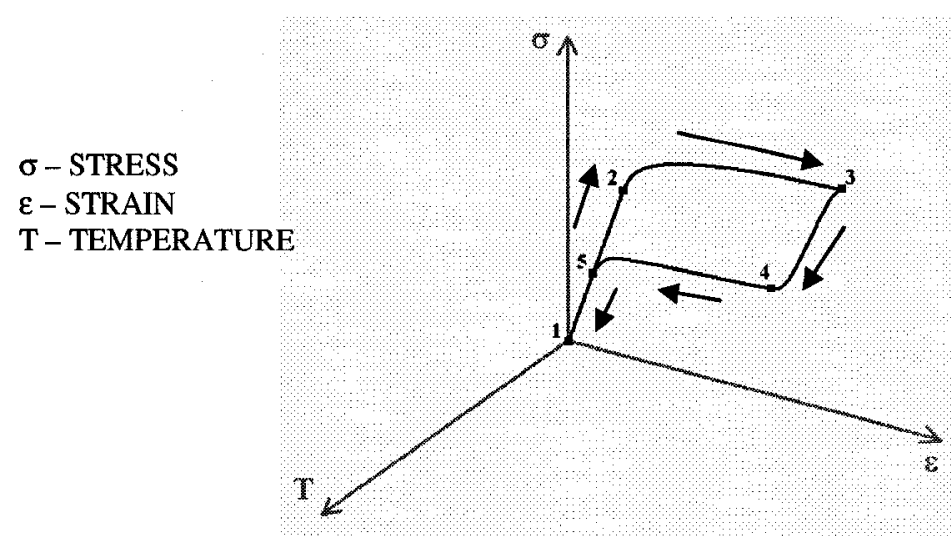

(c)

Figure 2.-Stress-strain-temperature curves for: (a) shape memory effect, (b) shape memory effect with residual stress, (c) superelasticity.

Figure 2 demonstrates the behaviour of the shape memory alloy during various transformation processes. Figures 2(a) and 2(b) represent the shape memory effect, while figure 2(c) represents superelasticity.

In figure 2(a) the material follows an initial linear elastic stress-strain curve from 1 to 2 before beginning the transformation to a deformed configuration at 3 (although no plastic deformation has occurred); the material is unloaded from 3 to 4 with a residual strain representing the deformation; heat is applied and the temperature rises with no strain recovery until a critical temperature is reached at 5 ; transformation to austenite 
occurs as the temperature is increased between 5 and 6 , returning the material to its previous geometry.

Figure 2(b) represents a similar process but with some applied stress maintained. Again the material is loaded in the linear elastic region from 1 to 2 and then deformed until reaching 3; an incomplete unloading occurs from 3 to 4 with some stress remaining; temperature is again increased until transformation to austenite occurs between 5 and 6 , recovering the previous geometry; the material is cooled from 6 to 7 until reaching another critical temperature at which it begins transformation back to martensite; however due to the applied stress the material strains to the deformed configuration until reaching 8 as it cools to ambient temperature.

Figure 2(c) demonstrates the superelasticity phenomenon. The material is loaded from 1 to 2 and transforms to the deformed configuration from 2 to 3 . As it is unloaded from 3 to 4 the material behaves like a linear elastic solid. When reaching a critical stress level at 4 the shape memory alloy transforms back to the previous geometry until reaching 5 and completely unloading.

\subsection{Shape Memory Alloy Model}

Modeling of the SMA actuator wire was based upon the Liang \& Rogers [22] SMA model. Since the SMA actuator was a wire, it could be considered one-dimensional, and hence any strain considered simply a change in length. The relationship between stress and strain could be written similar to Hooke's Law, and is referred to as the Constitutive Equation, equation 1. 


$$
\Delta \sigma=E(\xi) \Delta \varepsilon+\Omega(\xi) \Delta \xi+\Theta(\xi) \Delta T
$$

Eq. 1

The Young's modulus, $E(\xi)$, transformation tensor, $\Omega(\xi)$, and thermal expansion modulus, $\Theta(\xi)$, were all functions of the martensite fraction, $\xi$. These functions were given by equations 2 to 4 . The subscripts $A$ and $M$ represent the austenite and martensite phases respectively.

$$
E(\xi)=(1-\xi) E_{A}+\xi E_{M}
$$

Eq. 2

$$
\Omega(\xi)=-E(\xi) \varepsilon_{L}
$$

Eq. 3

$$
\Theta(\xi)=E(\xi)\left[(1-\xi) \alpha_{A}+\xi \alpha_{M}\right]
$$

Eq. 4

Therefore in addition to strain developed from applied stress and temperature changes, there is a compressive strain associated with the transformation between austenite and martensite. The maximum value for this strain is given by $\varepsilon_{\mathcal{L}}$, the maximum residual strain.

The martensite fraction, $\xi$, could be defined by another set of equations, referred to as the Phase Transformation equations. Phase transformations occurred in two directions, from martensite to austenite, or from austenite to martensite. Both transformations occurred only during a specific set of conditions, dependent upon the temperature and stress. 
Transformation from martensite to austenite occurred when the SMA was heated from the austenite start temperature, $A_{s}$, to the austenite finish temperature, $A_{f}$. After surpassing $A_{f}$, the SMA was entirely in the austenite phase. This transformation was governed by equation 5 .

$$
\xi=\left(\frac{\xi_{0}}{2}\right)\left\{\cos \left[a_{A}\left(T-A_{s}-\frac{\sigma}{C_{A}}\right)\right]+1\right\}, \quad A_{s}^{\prime}<T<A_{f}^{\prime}
$$

Eq. 5

The coefficient, $a_{A}$, in equation 5 must satisfy the condition that $\xi=\xi_{0}$ when $T=$ $A_{s}$ and $\xi=0$ when $T=A_{f}$. Therefore, $a_{A}$ is given by equation 6 .

$$
a_{A}=\frac{\pi}{A_{f}-A_{s}}
$$

Eq. 6

The transformation temperatures themselves are dependent upon stress through a linear relationship according to equations 7 and 8 , where $C_{A}$ is the stress influence coefficient for the austenite phase.

$$
\begin{aligned}
& A_{s}^{\prime}=A_{s}+\frac{\sigma}{C_{A}} \\
& A_{f}^{\prime}=A_{f}+\frac{\sigma}{C_{A}}
\end{aligned}
$$


Transformation from austenite to martensite occurred when the SMA was cooled from the martensite start temperature, $M_{s}$, to the martensite finish temperature, $M_{f}$. Once cooled beyond $M_{f}$, the SMA was entirely in the martensite phase. Transformation in this region was governed by equation 9 .

$$
\xi=\left(\frac{1-\xi_{0}}{2}\right) \cos \left[a_{M}\left(T-M_{f}-\frac{\sigma}{C_{M}}\right)\right]+\left(\frac{1+\xi_{0}}{2}\right), \quad M_{f}^{\prime}>T>M_{s}^{\prime}
$$

Eq. 9

The coefficient, $a_{M}$, must satisfy the condition that $\xi=\xi_{0}$ when $T=M_{s}$, and $\xi=1$ when $T=M_{f}$. Therefore, $a_{M}$ is given by equation 10 .

$$
a_{M}=\frac{\pi}{M_{s}-M_{f}}
$$

Eq. 10

The transformation temperatures are again dependent upon stress, and given by equations 11 and 12 , where $C_{M}$ is the stress influence coefficient for the martensite phase.

$$
\begin{aligned}
& M_{s}^{\prime}=M_{s}+\frac{\sigma}{C_{M}} \\
& M_{s}^{\prime}=M_{s}+\frac{\sigma}{C_{M}}
\end{aligned}
$$

Eq. 11

Eq. 12 
It was also important to note that the initial martensite fraction, $\xi_{0}$, is a constant throughout an entire transformation region. Once a transformation ends, the final value of the martensite fraction becomes the initial value of $\xi$ for the next transformation. Furthermore, there is no phase transformation if the temperature is not within either set of transformation temperatures. Hence, the martensite fraction remains a constant.

\subsection{Shape Memory Alloy Applications}

\subsubsection{SMA Actuated Microvalves}

The Biorobotics Group at Case Western Reserve University in Cleveland, Ohio, is developing robots that mimic crickets, as shown in figure 3 . Actuation of the robotic appendages is powered using a miniature pneumatic system [3].

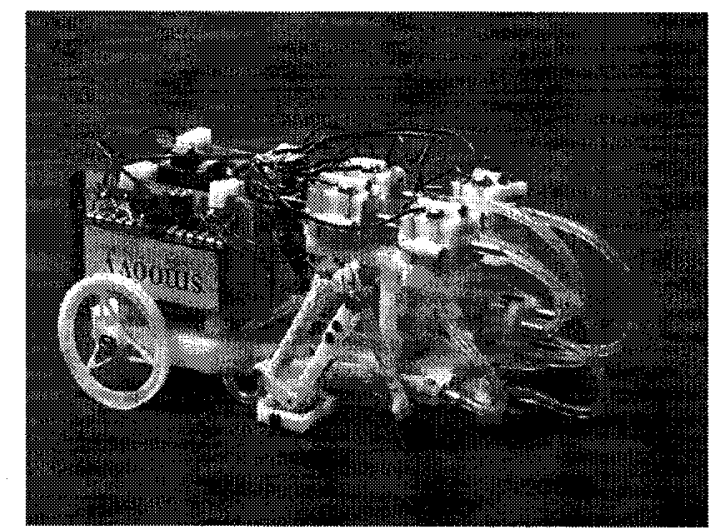

Figure 3.-CWRU Biorobotics Autonomous Hybrid Robot [3].

Notably, the pneumatic system incorporates shape memory alloy actuated microvalves. The microvalve design uses a spring component to act as a bias closing 
force, and an SMA actuator to generate an opening force. Fluid flow rate is proportional to the applied current in the SMA. Figure 4 illustrates the overall concept and the prototype. The valve is manufactured by sputtering nickel and titanium on a silicon substrate.

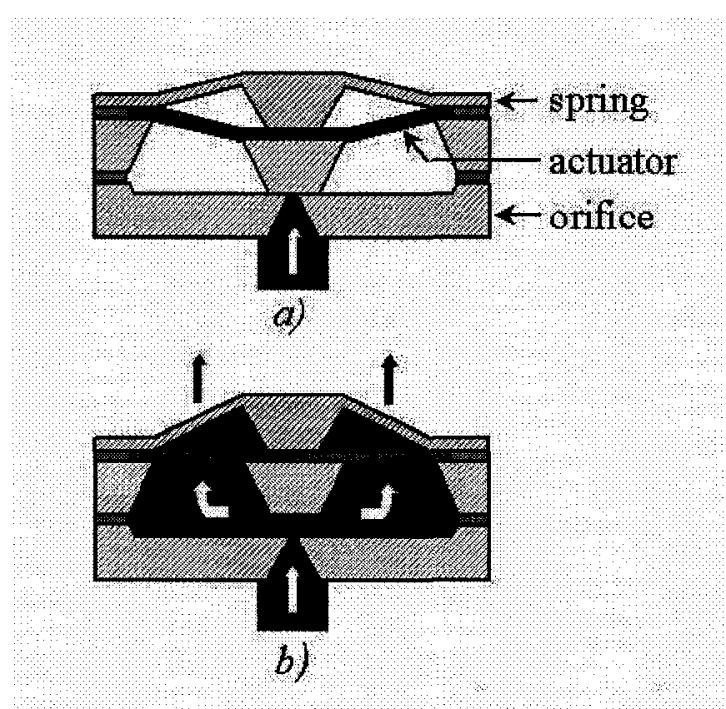

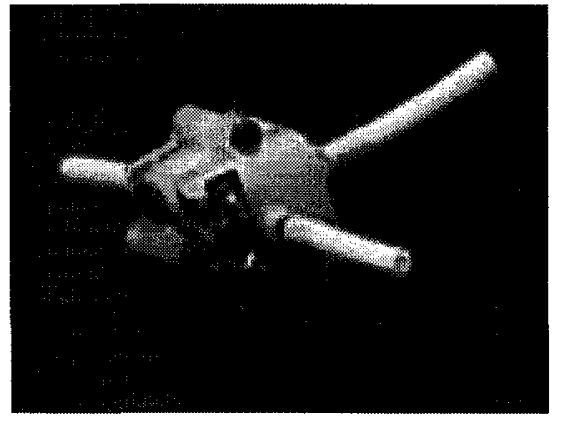

(c)

Figure 4.-CWRU Microvalve concept, (a) closed, (b) open, (c) prototype [3].

A second microvalve concept has been designed as part of the European BriteEuram project, SMA micro Actuators for Medical Applications (SAMA) at the Katholieke Universiteit Leuven (KUL) in Heverlee, Belgium. The microvalve design, shown in figure 5, is for use in an implantable drug delivery system [4]. The pinch valve, composed of silicon rubber, opens through the heating of a shape memory alloy wire actuator. The contraction of the flexture causes the valve to release the contents of the system. Electronic components are easily integrated into the component. Furthermore, its simplicity makes it ideal for mass production. 


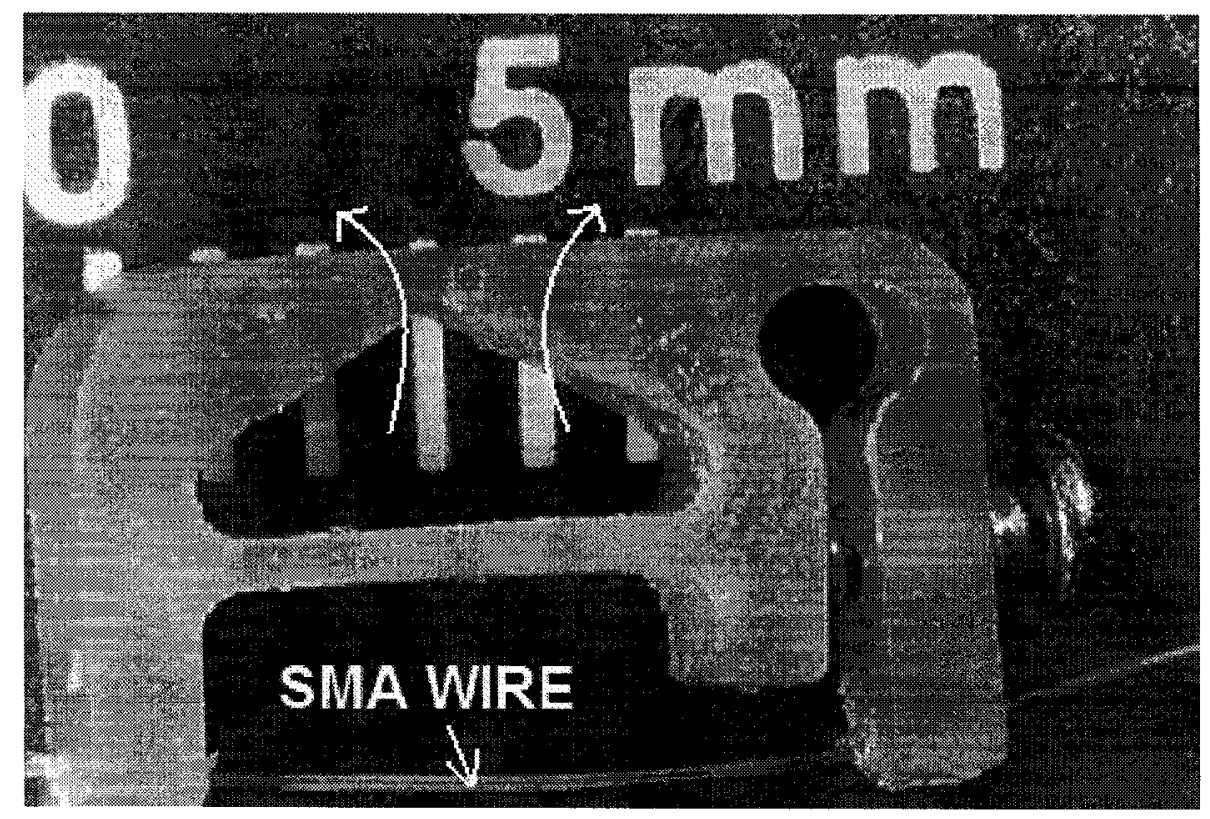

Figure 5.-KUL implantable drug delivery system microvalve design [4].

\subsubsection{Miniature SMA Actuated Grippers}

Research on shape memory alloy actuators at the University of Tokyo has led to the development of a miniature SMA actuated clean gripper. The gripper, as shown in figure 6 , is designed for use in applications where delicate and precise handling is required [5]. Actuation is accomplished using a set of antagonistic SMA wire actuators for each cantilevered finger. Although the gripper was tested only using one actuated finger, force and position control was successfully accomplished. 


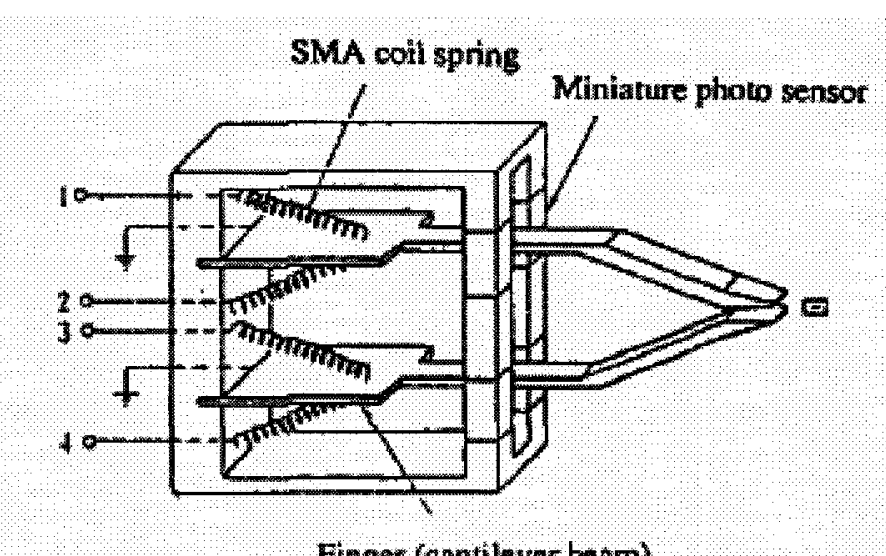

Finger (cantilever beam)

Figure 6.-University of Tokyo SMA actuated clean gripper [5].

A microscale shape memory alloy actuated gripper for miniaturized manufacturing applications is being developed at the Institute of Microtechnology in Braunschweig, Germany. Fabricated from a $360 \mu \mathrm{m}$ thick wafer of silicon, the gripper has flexural hinges and employs an antagonistic set of SMA foil actuators to open and close [6]. The actuators themselves have a thickness of $50 \mu \mathrm{m}$. Figure 7 shows the design of the gripper and SMA actuators.
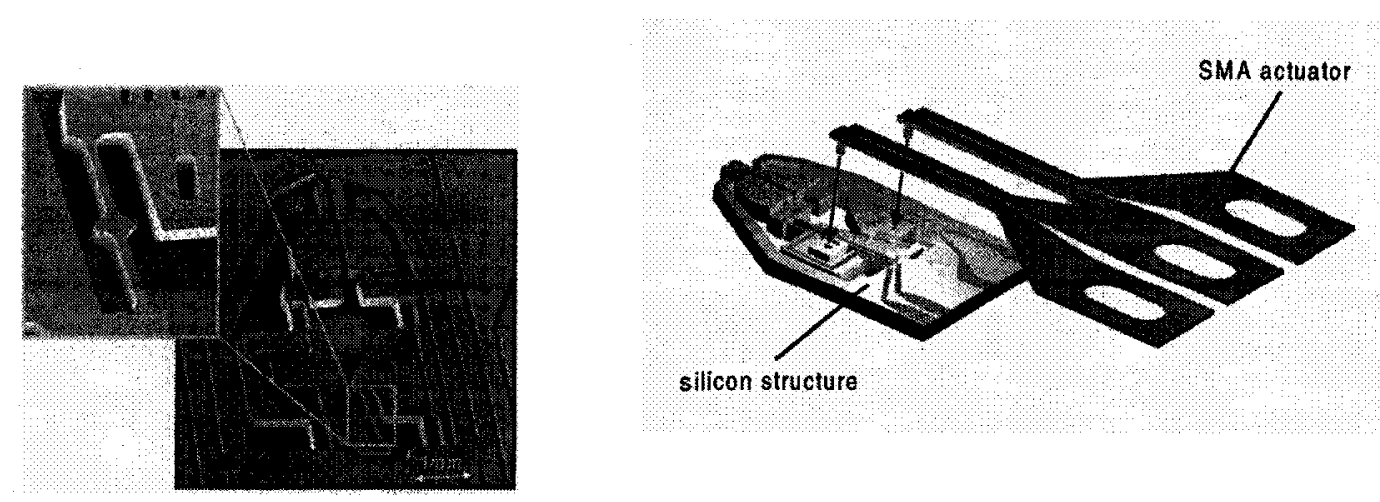

Figure 7.-Institute of Microtechnology SMA actuated gripper [6]. 


\subsubsection{SMA Actuator for Tactile Information Display}

Shape memory alloys are used in the design of an actuator for tactile information display (such as Braille) typically used by the visually impaired. Research performed at the Laboratoire de Robotique de Paris shows the feasibility of using SMA spring actuators to displace an array of miniature pins [7]. Figure 8 demonstrates the concept. Two SMA springs are arranged antagonistically to allow actuation in both directions. Figure 9 shows a prototype of the actuator and a concept for an 8x8 display grid.

The use of an array of display grids allows the user to read multiple lines of text at once after which the system may be commanded to display the next lines. Shape memory alloys make the actuator low cost and easy to integrate into arrays.

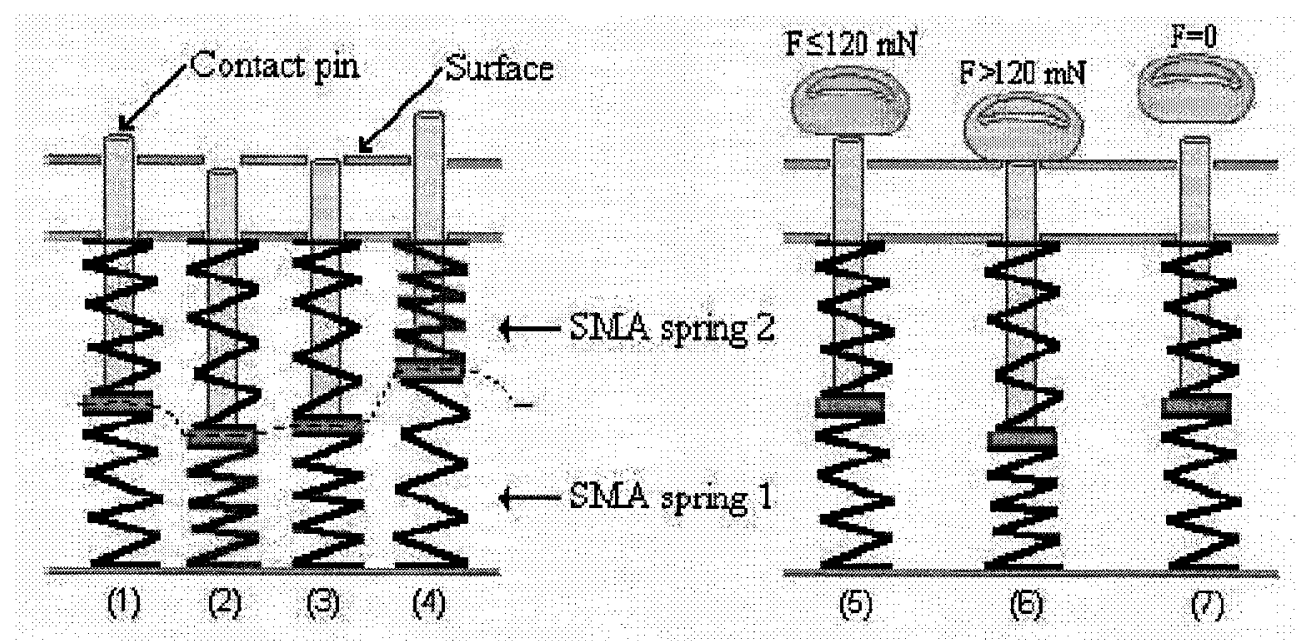

Figure 8.-Laboratoire de Robotique de Paris SMA actuator concept [7]. 

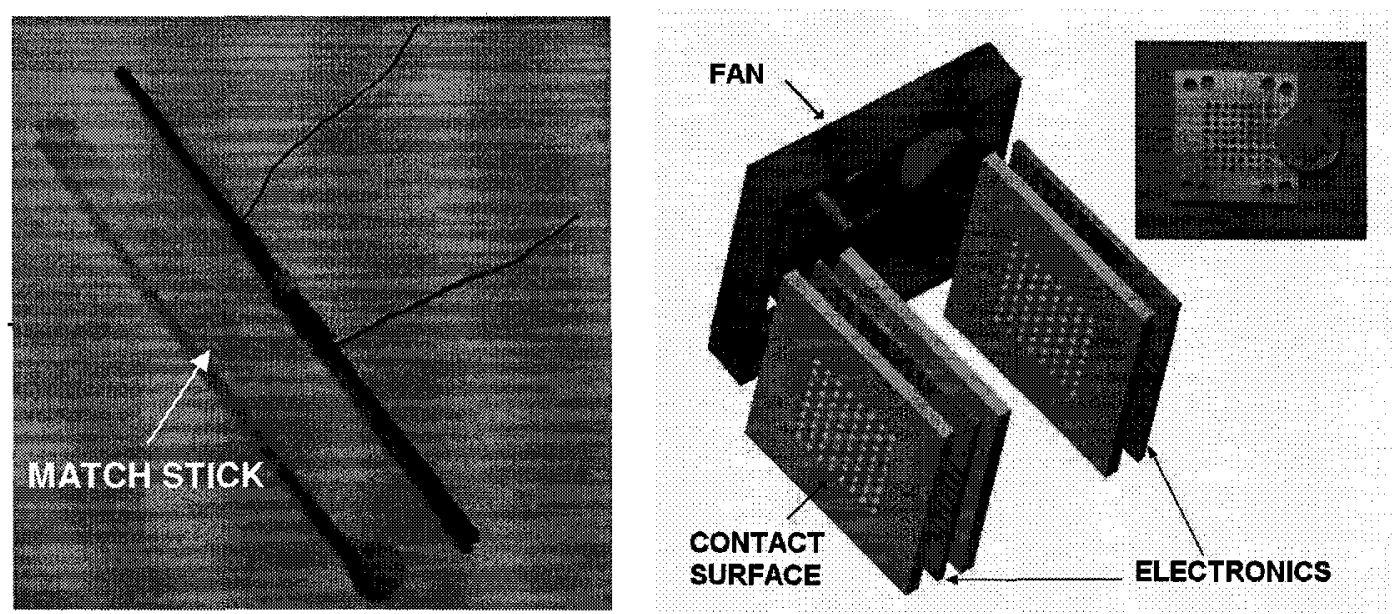

Figure 9.-Laboratoire de Robotique de Paris SMA actuator prototype and $8 x 8$ display grid concept [7].

\subsubsection{Miniature Rotary SMA Latching Device}

Developed for the Goddard Space Flight Centre by the Johns Hopkins University Applied Physics Laboratories, the simple rotary shape memory alloy device depicted in figure 10 may serve a wide range of applications [8]. An actuator composed of a strip of shape memory alloy is twisted by a prescribed angle and inserted into an assembly loaded by a bias torsional spring. When heated, the SMA actuator counteracts the torsional spring and turns the latch pin.

This latching device is designed for use in space applications, such as the separation of spacecraft components or the release of appendages. 

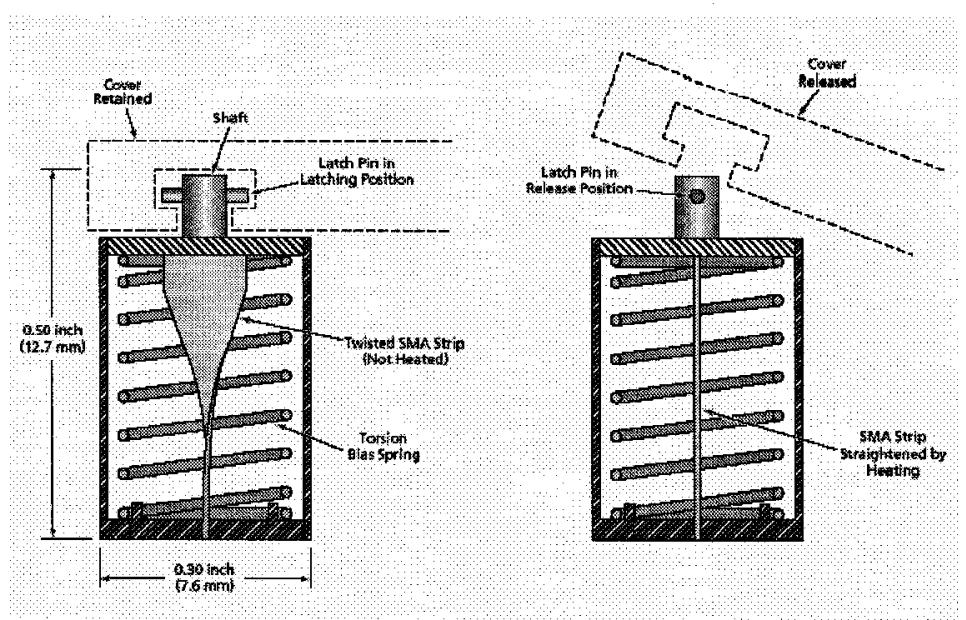

Figure 10._Miniature SMA rotary latching device [8].

\subsubsection{Modular Machine using Rotary SMA Actuators}

The Distributed Systems Design Research Group at the National Institute for Advanced Industrial Science and Technology in Japan is developing modular robotic components using miniature shape memory alloy actuators [9]. The modular design will allow the machine to actively change its configuration in order to explore hazardous environments or manipulate objects. Figure 11 demonstrates the concept of two components interacting.
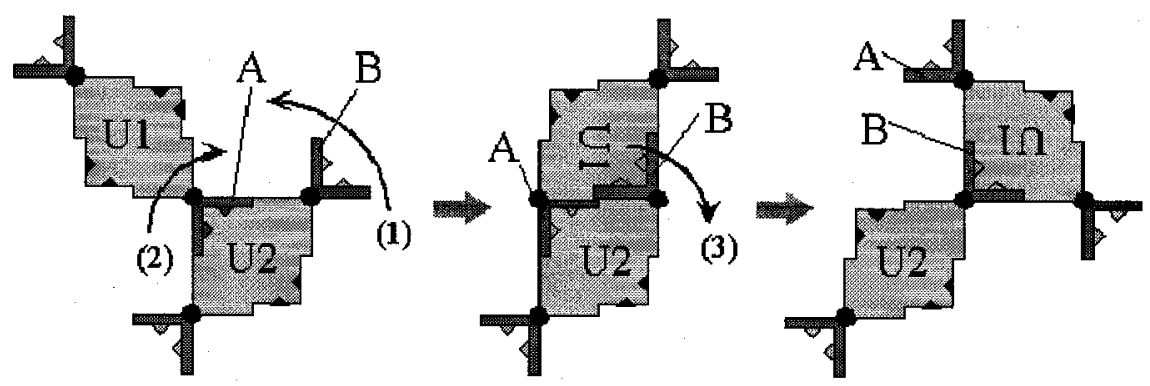

Figure 11.-DSYSD modular machine concept [9]. 
The male connectors, $\mathrm{A}$ and $\mathrm{B}$, are actuated using preloaded rotary shape memory alloy torsional springs. Each actuator is composed of two antagonistic SMA springs, as illustrated in figure 12.
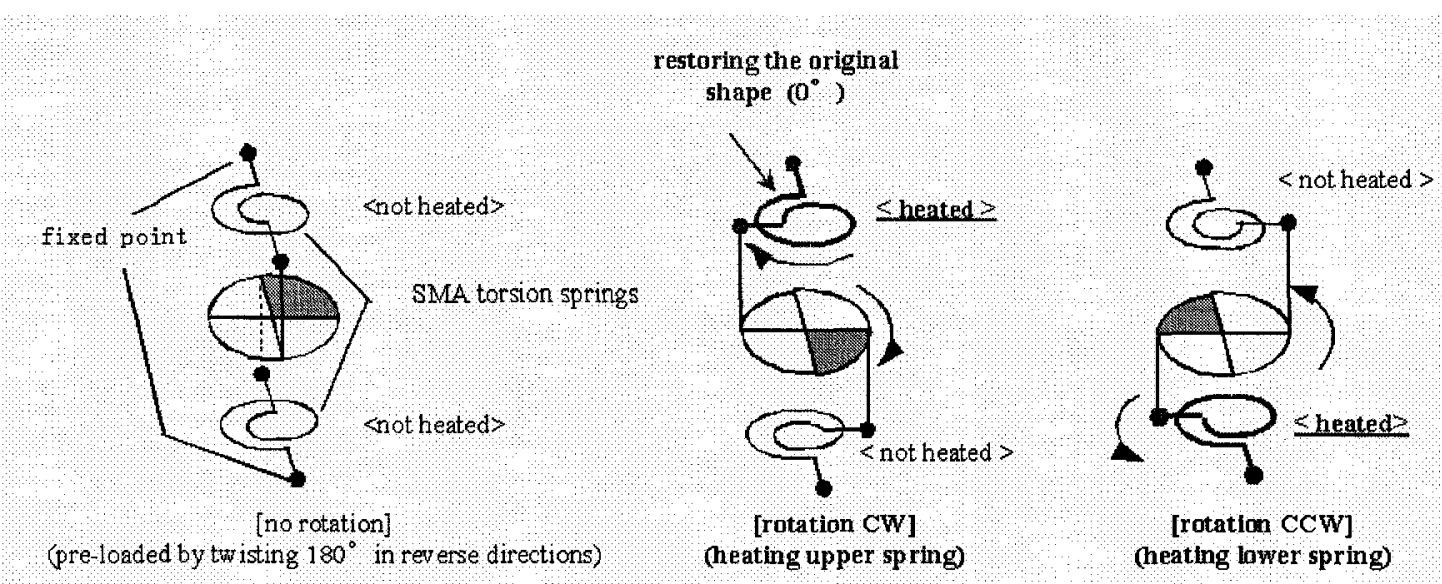

Figure 12.-Operation of the rotary SMA actuator [9].

\subsection{Actively Controlled Rotor Blades}

\subsubsection{Smart Material-Actuated Rotor Technology (SMART)}

Research performed at the Boeing Company has focused on implementing smart materials into helicopter rotor blades for the purpose of vibration attenuation as well as blade tracking [10]. The MD900 helicopter blade was modified to include a trailing edge flap actuated by piezoelectric elements, as well as a trim tab actuated by shape memory alloy. Figure 13 shows the concept for the full scale rotor blade and cross-section. 


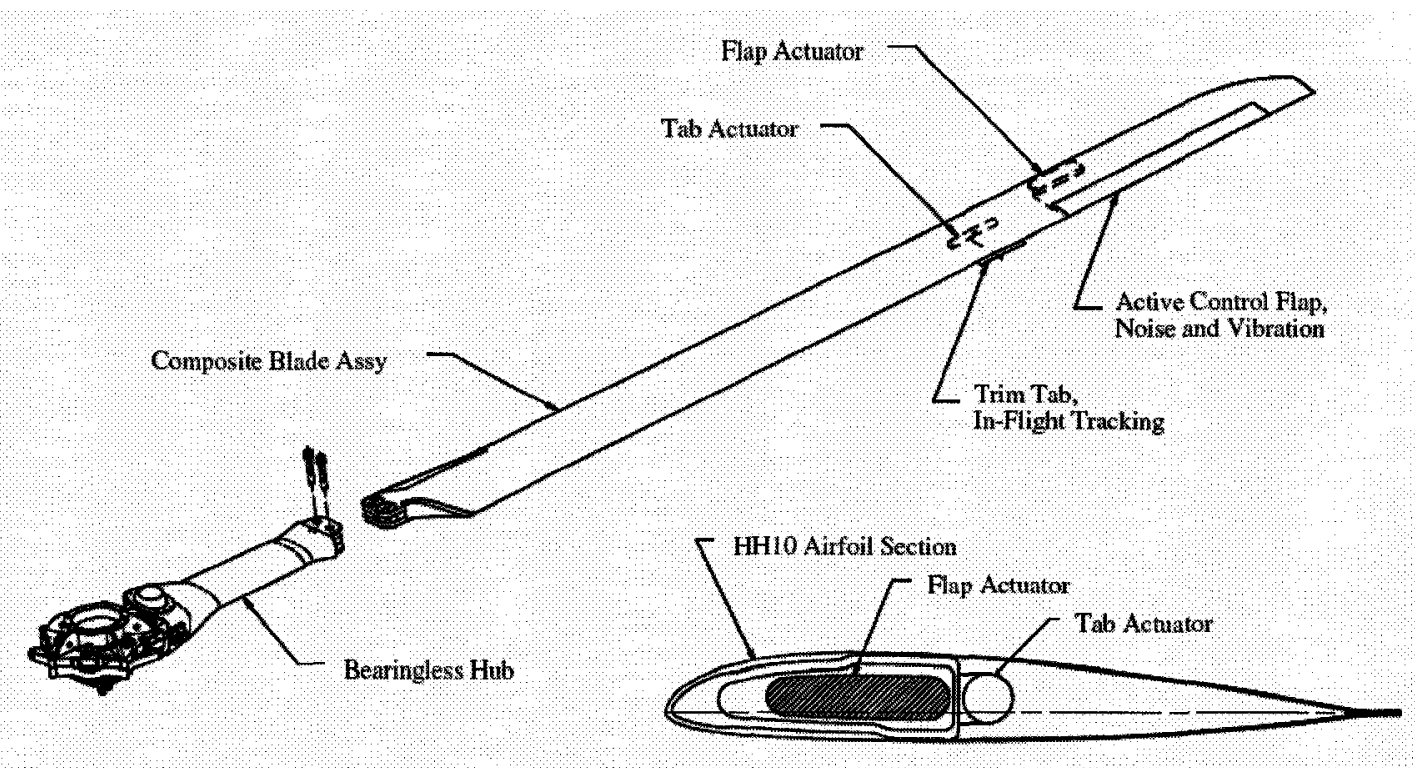

BLADE CROSS-SECTION

Figure 13.-Boeing SMART rotor blade concept [10].

Actuators for both the trailing edge flap and trim tab were developed. The systems were then independently tested at the bench level. The trailing edge flap actuator employs a set of two piezoelectric stacks acting biaxially to bend a titanium flexure attached to the outboard mount. This amplifies the displacement capabilities of the piezoelectric stacks while still producing satisfactory force. The trim tab actuator uses a set of shape memory alloy torsion tubes to produce an angular displacement. An active locking mechanism maintains the desired position with another set of shape memory alloy elements.

Both active systems met the requirements of actuation stroke and force. Although no wind tunnel or whirl tower testing was performed, aeroelastic simulations suggested that the actively controlled rotor system would greatly improve the aerodynamic performance of the blades and lead to many benefits such as improved part life. Both trailing edge flap and trim tab actuator designs are shown in figure 14. 


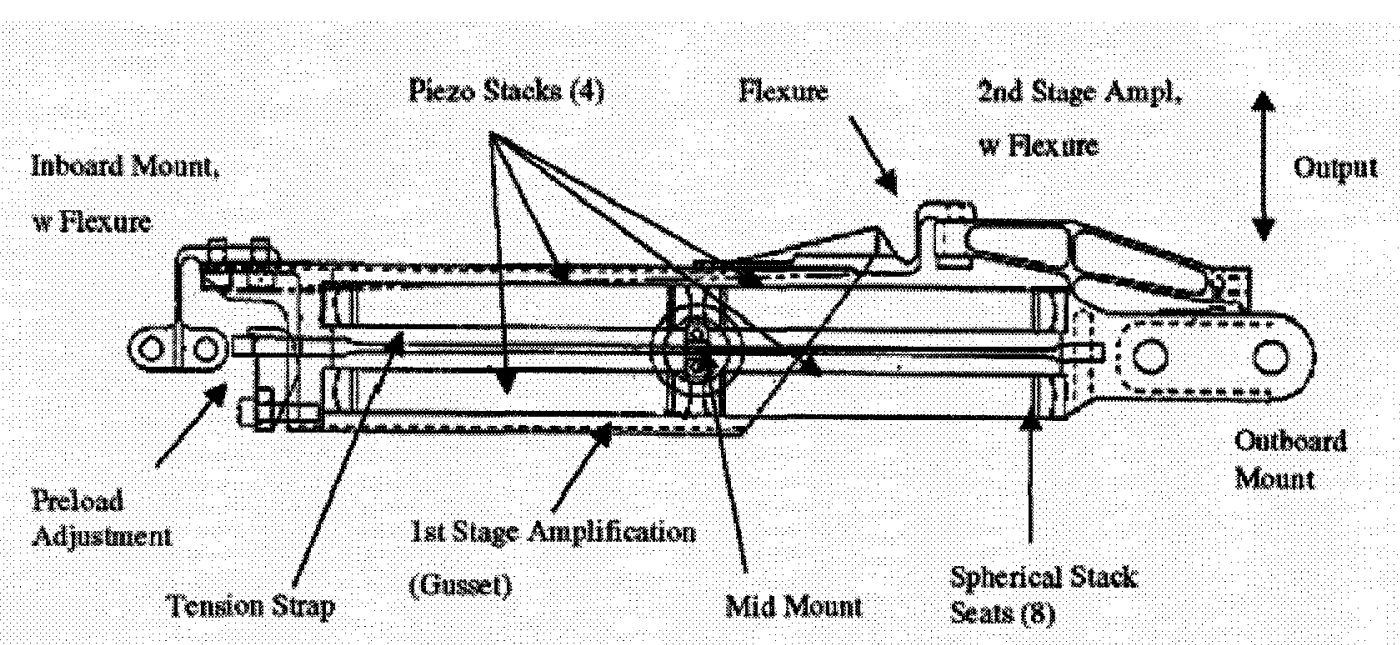

(a)

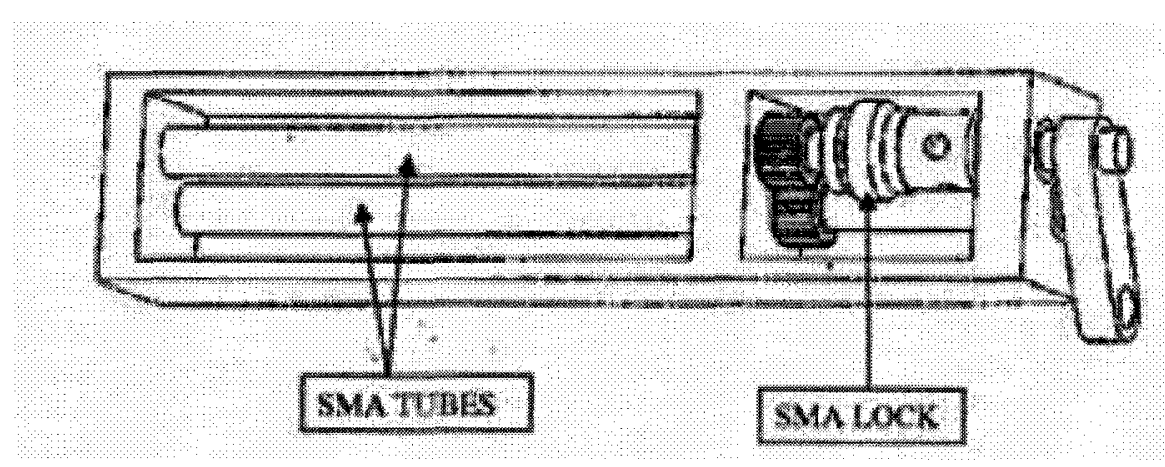

(b)

Figure 14.-SMART rotor blade actuation mechanisms for (a) trailing edge flap and (b) trim tab [10].

\subsubsection{Helicopter Blade with Integrated Smart Morphing Actuator}

An actively controlled helicopter blade tip has been developed at the University of Rome III in collaboration with the Italian Aerospace Research Centre (IARC) and University of Palermo in Italy. The blade tip is deflected downward through the combined use of a magneto-rheological device as shown in figure 15. A shape memory 
alloy based actuator and a set of concentrated masses arranged along the span-wise direction of the tip [11].

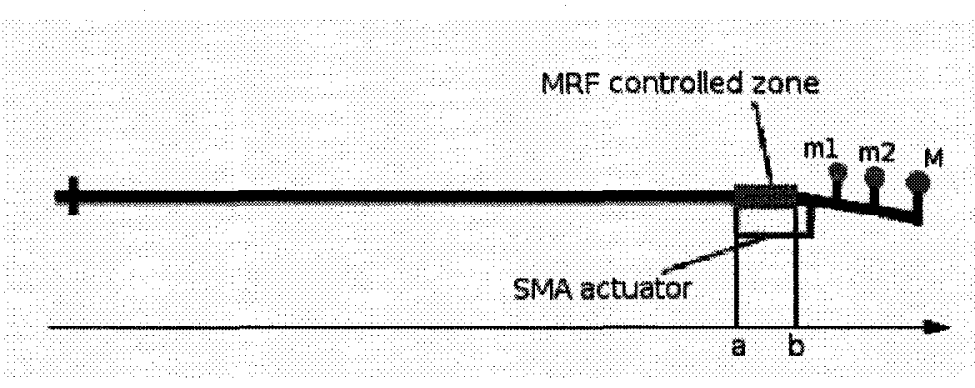

Figure 15._IARC actively controlled rotor blade tip concept [11].

The moment for deflecting the tip is provided primarily by the centrifugal loads created by the concentrated masses. The magneto-rheological dampener is used to alter the stiffness properties of the blade near the tip, providing compliance during actuation. The shape memory alloy actuator is then employed for position control.

The focus of this work was numerically investigating the aeroelastic response of the blade and the feasibility of the concept altogether. It was found that the actuation and deflection of the tip was successful using this combination of devices. Vibration loads were reduced (except for the out-of-plane loads which increased by a factor of four) and the aeroelastic stability of the blade was maintained. 


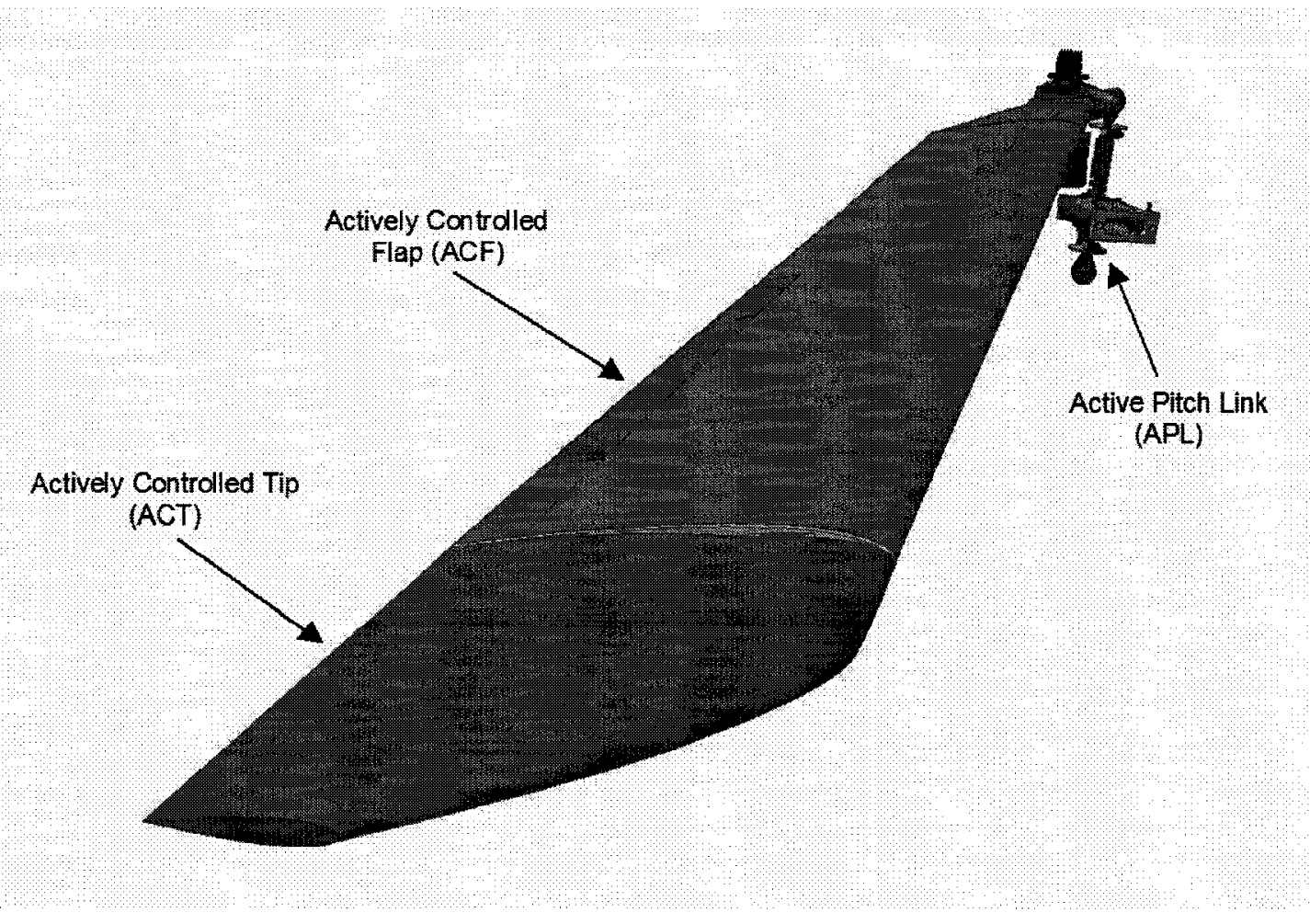

Figure 16.-SHARCS rotor blade with ACT, ACF, and APL subsystems [12].

\subsubsection{Smart Hybrid Active Rotor Control System (SHARCS)}

The Smart Hybrid Active Rotor Control System (SHARCS) introduces a scaled concept for a rotor blade embedded with multiple active control systems. Three separate systems are incorporated into the design: the active pitch link (APL), the actively controlled flap (ACF), and the actively controlled tip (ACT). Vibration reduction is primarily achieved using both the APL and ACF, while acoustic noise reduction is primarily achieved using the ACT. Figure 16 demonstrates the SHARCS concept.

The APL is a closed-loop active control mechanism based upon a smart spring concept [13]. By using piezoelectric actuators, the stiffness and dry friction of the APL may be varied to adapt its structural response. Thus, the structural properties of the rotor 
blade—stiffness, damping, and effective mass—may be varied as well (since the APL acts as a boundary condition). The extraction of energy through damping in the APL reduces vibration [14]. Noise reduction using the APL may also be possible by superimposing pitch control inputs of $1^{\circ}$ amplitude. Figure 17 demonstrates the conventional pitch link (a) and Active Pitch Link (b).

The ACF is a flow control device spanning from $65-85 \%$ radius and covering $15 \%$ of the chord length on the trailing edge. Aerodynamic properties of each blade can then be altered to achieve reduction of noise [15] and vibration [16] [17] [18] [19]. A high blade torsional stiffness enables the ACF to perform as a high-lift devicedownward flap deflections yield an increase in lift. Conversely, when the blade torsional stiffness is low, the ACF performs as a servo-tab-downward flap deflections cause a

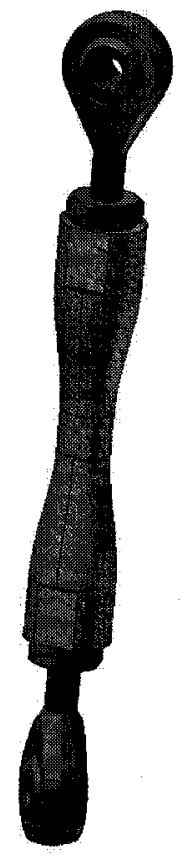

(a)

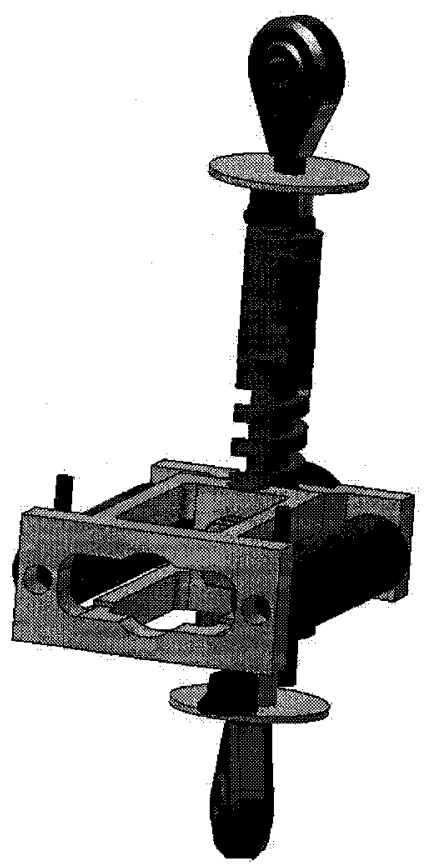

(b)

Figure 17.-(a) Conventional pitch link and (b) Active Pitch Link concept [12]. 
nose-down pitching motion of the blade (decrease in lift).

The ACT is an open-loop flow control device extending over the outermost $10 \%$ of the rotor blade. Individual blade tip anhedral angles may be adjusted by the pilot to reduce noise. A downward deflection of the blade tip increases the distance between the generated vortices and subsequent blades-decreasing the effects of BVI. The anhedral deflection also results in a redistribution of lift along the tip; hence, the tip vortex strength is reduced.

\subsection{Objectives}

The goal of the research presented in this thesis is to investigate the feasibility of shape memory alloy wires for antagonistic angular position control of a shaft. Casual control algorithms were conceived and tested for both a single actuator wire and dual actuator wires acting upon the shaft. It was assumed that linear-time-invariant control theories could not be applied due to the non-linear behaviour of shape memory alloy. However, PID control and Ziegler-Nichols tuning methods were attempted. A model of the shape memory alloy actuated shaft was constructed for both the single channel and dual channel cases. Comparison between the experimental results and model results are presented for validation.

Research into this application of shape memory alloy is motivated by the requirement of an actuation system for a helicopter rotor blade tip flap for the SHARCS (Smart Hybrid Active Rotor Control System) project. The SHARCS project aims to attenuate both noise and vibration on helicopters using multiple active systems installed 
on each rotor blade. One system, the actively controlled tip (ACT) flap, will serve as a static device, deflecting the outermost $10 \%$ of the blade downward to achieve an anhedral angle of $0-20^{\circ}$. The purpose of the ACT is to decrease the effects of blade vortex interaction (BVI) by both increasing the miss distance between the tip vortex and succeeding blade (avoiding a collision) and by decreasing the strength of the vortex itself. Such a system may be an ideal application of shape memory alloy for actuation. However, it will also be shown that the aerodynamic and centrifugal loads acting upon the tip flap are too large for a shape memory alloy based actuator at the scale of the SHARCS blade. 


\section{Experimental Investigations}

\subsection{Apparatus}

The research and development of a controller for an SMA based hinge actuation system was conducted at the bench level. Figure 18 depicts the setup in flow chart. A rotary shaft, supported by a set of bearings, was used to represent a flap hinge. Angular position of the shaft was measured by a potentiometer. Thermocouples were used to

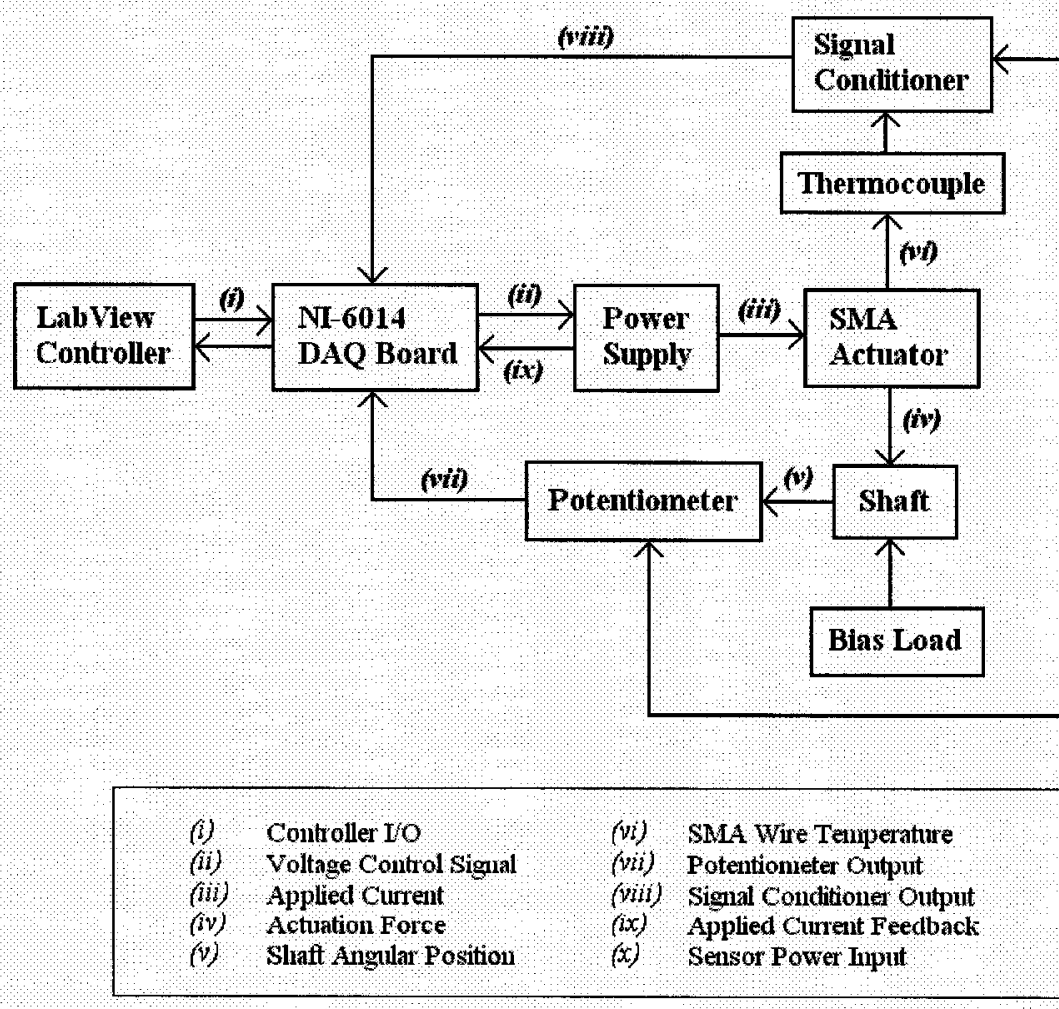

Figure 18._Flow chart representation of experimental setup. 
measure the temperature of each SMA wire-signal conditioners cleaned and amplified the thermocouple readings. The current through each SMA wire was maintained and controlled by a custom power supply built in-house. All sensors were read using a data acquisition system and transferred to digital data on a computer. Current from the power supply was controlled in real time from the same computer, either in open loop or closed loop control. Furthermore, bias torque loading of the shaft was provided by weights attached to a pulley system. Figure 20 shows the setup arranged for dual channel control.

\subsubsection{Flexinol Shape Memory Alloy Actuator Wire}

Flexinol, manufactured by Dynalloy Inc., is a commercialized brand of Nitinol, a shape memory alloy constituting of Nickel and Titanium [20]. The Flexinol wires purchased for this research have a diameter of $0.015 "(0.381 \mathrm{~mm})$. Each wire is capable of carrying up to approximately $20 \mathrm{~N}$ of tension. Although the ultimate tensile strength of the Flexinol wire is much greater, the applied force must be limited otherwise the shape memory properties of the material will be destroyed. The Flexinol wires must also be cycled or trained in order to reach a steady state response. While applied stress and temperature may have an effect, the typical lifetime of Flexinol wires is hundreds of thousands of cycles.

\subsubsection{Potentiometer}

A Vishay Spectral Model 157 Precision Industrial Potentiometer with a resistance of $5 \mathrm{k} \Omega$ was used. The shaft was turned down on a lathe at one end to match the diameter of the potentiometer. The shaft and potentiometer were then connected via a tight fitting 
piece of plastic tubing. Due to the interference of the tubing with the shaft and potentiometer, static friction maintained a tight connection hence guaranteeing no slip and an accurate shaft angle measurement. An excitation signal of $5 \mathrm{~V}$ was provided by an AC-DC adaptor. The potentiometer output signal ranged between $0 \mathrm{~V}$ and $5 \mathrm{~V}$, and hence a scaling factor of 72 was used to represent the shaft angular position in degrees. A discontinuity existed when travelling from the $5 \mathrm{~V}$ position to the $0 \mathrm{~V}$ position and vice versa. In order to avoid possible problems due to this discontinuity, the potentiometer was arranged such that the base reference position gave an output of $2.5 \mathrm{~V}$, hence allowing a half rotation in either direction.

\subsubsection{Thermocouples}

Type-K thermocouples (Alumel and Chromel junction), attached to OM5-ITC-K signal conditioners, were purchased from Omega. The same AC-DC adaptor used to excite the potentiometer was used to excite the thermocouple signal conditioners. Initial calibration was carried out to determine the validity of the data sheets provided by the manufacturer. An ice bath of deionized water provided a reference point of approximately $0{ }^{\circ} \mathrm{C}$ observed as a constant voltage region during melting. Figure 19 shows the signal conditioner output voltage during this process. The thermocouple signal conditioners were then tested at various ambient temperatures to ensure the correct operation of the cold junction compensation circuit. Based upon the manufacturers calibration data, the thermocouple temperature was assumed to be a linear function of the signal conditioner voltage. The offset value, $\beta$, was determined from the ice bath and the proportionality value, $\alpha$, was derived from the manufacturer's calibration data sheet. 


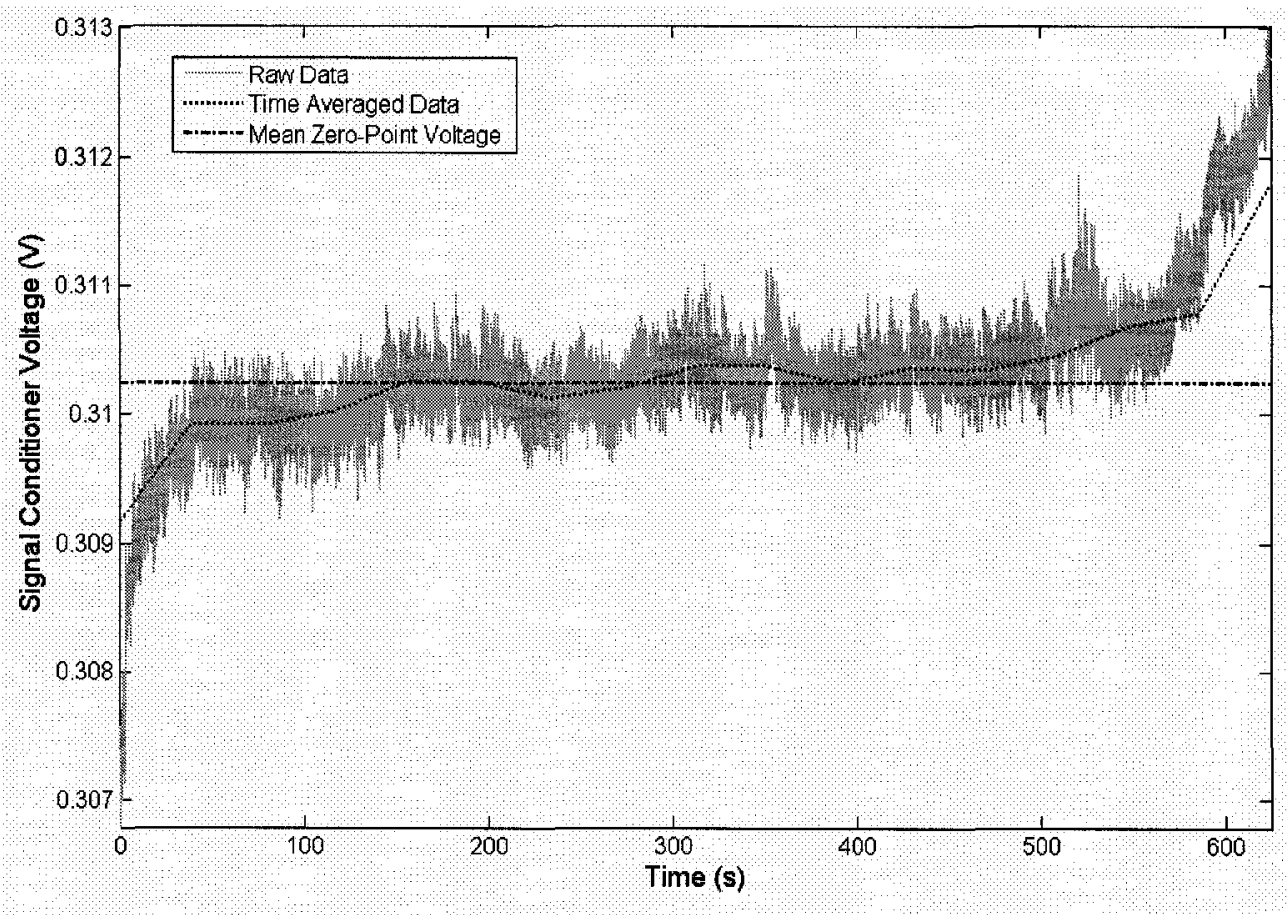

Figure 19.-Thermocouple signal conditioner calibration data.

Equation 13 represents the thermocouple temperature as a function of the signal conditioner voltage.

$$
T=\alpha V+\beta
$$

Eq. 13

Each thermocouple was attached to the respective SMA wire by simply twisting the alumel and chromel wires together to form a junction, and subsequently twisting the junction around the SMA wire. The free ends of the alumel and chromel wires were then wound around copper leads attached to the signal conditioners. Similar to the potentiometer, the output signals ranged between $0 \mathrm{~V}$ and $5 \mathrm{~V}$. 


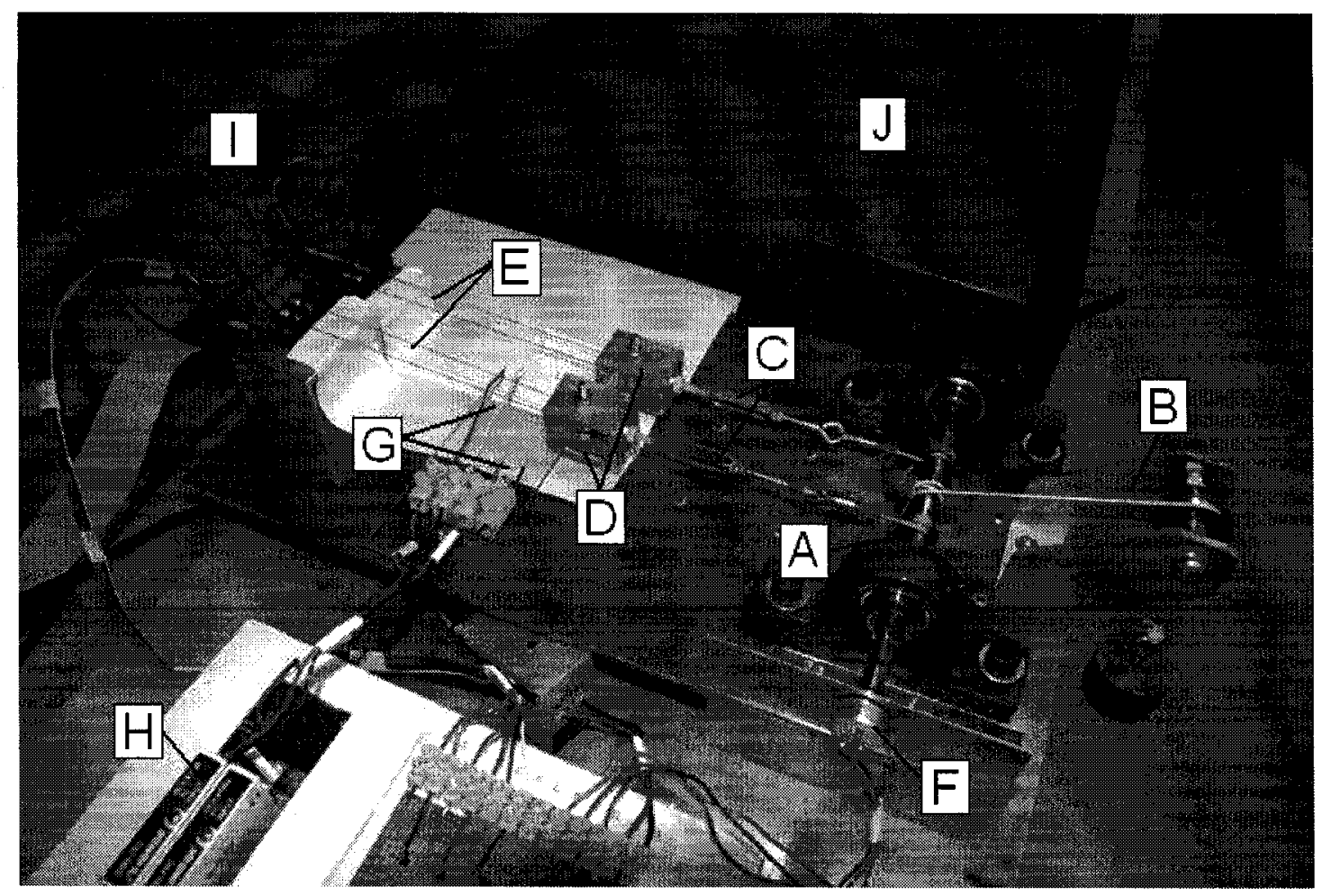

Figure 20.-Experimental setup: A. shaft; B. mass \& pulley system; C. turnbuckles; D. pulley blocks; E. SMA wires; F. potentiometer; G. thermocouples; H. signal conditioners; I. DAQ board; J. power supply.

\subsubsection{Power Supply}

Electrical resistive heating of the SMA actuator wires was accomplished by applying a controlled amount of current to each wire. Output from the data acquisition system was limited to proportional voltage control therefore a custom power supply was constructed by laboratory supervisor Gary Clements. Voltages across a pair of $1 \Omega$ resistors were controlled directly by the user through the data acquisition system. From Ohm's law, the current through each resistor was proportional to its applied control voltage. Each SMA wire was then connected in series with the respective resistor and hence yielded a current proportional to the voltage applied across the resistor. 


\subsubsection{Bias Load}

A small frame with a pulley was machined and attached to the end of the apparatus, with the pulley hinge arranged parallel to the shaft. Bias moment loads were then applied by hanging masses linked through a string to the shaft. The load direction was determined by winding the string around the top or bottom of the shaft. Bias loads were typically only used during experiments using a single SMA wire, therefore the tension in the actuator was determinate and equal to the weight hanging from the pulley. Four masses were machined from 1" and 2" diameter cylindrical steel stock. Through holes were drilled and tapped so that eye screws could be inserted, allowing multiple masses to be connected in series. Furthermore, a single mass was permanently fixed to the pulley string to maintain tension.

\begin{tabular}{|cc|}
\hline Specimen & Mass (g) \\
\hline I & 235.2 \\
II & 234.3 \\
III & 462.3 \\
IV & 462.0 \\
\hline
\end{tabular}

Table 1.-Bias load masses.

\subsubsection{Data Acquisition System}

Sensor data and output voltages were observed and controlled from a computer employing a National Instruments PC-6014 Data Acquisition Board. Interfacing between the user and the apparatus was implemented through National Instruments' software 
package, LabView 6.1. Input and output from the DAQ board was observed and controlled in open and closed loop methods. High-frequency noise was attenuated by inserting $10 \mu \mathrm{F}$ capacitors in parallel with the input terminals of the data acquisition board, acting as a low-pass filter.

\subsection{Methods \& Results}

\subsubsection{Open Loop Responses}

Initial experiments were conducted to characterize the behaviour of a single SMA actuator wire connected to the shaft and constrained at the other end of the apparatus using a screw terminal. A program was created in LabView to observe the SMA temperature, applied current and shaft angular position while also allowing the user control over the applied current. Data was sampled at $12000 \mathrm{~Hz}$, and averaged over blocks of 600 samples to reduce noise, leading to an effective sampling rate of $20 \mathrm{~Hz}$. Results of each effective sample were displayed on a graphical user interface. Output voltage from the data acquisition board was updated at each effective sample time from a slider on the interface, allowing the user real-time control of the applied current.

It was observed that small impulses of current, approximately 1-1.5 A applied for 1-2 seconds, were sufficient to fully contract the SMA wire. The full actuation was clearly observable from the change in shaft angle, which reached a limit after the SMA wire was fully heated. Further heating of the wire was therefore ineffectual. Passive conduction and convection around the wire was the only source of cooling which 


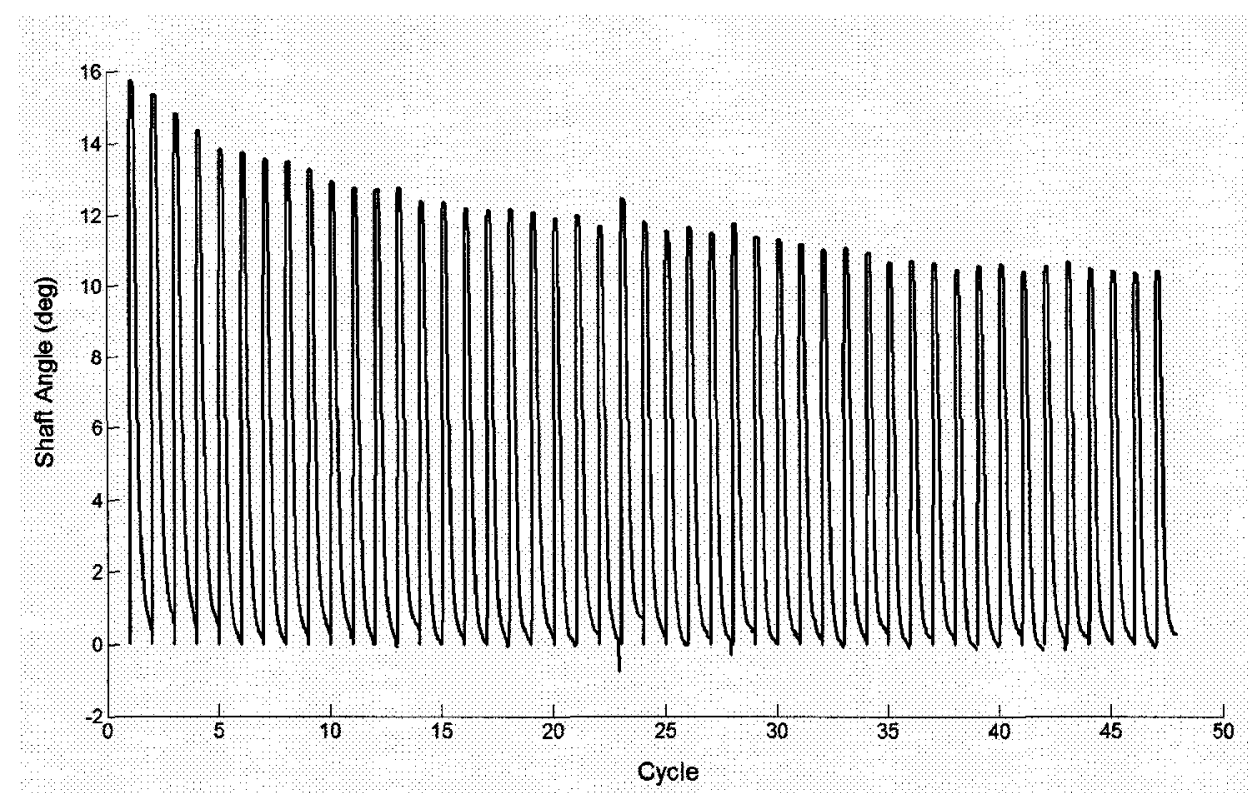

Figure 21.-SMA actuator wire cycling data.

returned the SMA wire to its original configuration-a process that required approximately 30 seconds.

Cycling (or 'training') of the SMA wires was required to achieve a steady repeatable response. This was conducted by applying a current of $1 \mathrm{~A}$ for 2 seconds and observing the response over 30 seconds. It was found that approximately 30 cycles of this actuation were required to reach the steady-state response of the SMA wire. This process was therefore conducted whenever a new SMA wire was used. Figure 21 shows the SMA wire reaching a steady state response through cycling.

The response of the SMA wire was then observed using various bias loads as shown in figure 22. The weight on the pulley was increased incrementally. At each loading condition, the SMA wires were subjected to an applied current of $1.5 \mathrm{~A}$ for 3 
seconds. The response was then observed for 30 seconds. The recorded data was then analyzed to examine the effect of varying stress on the SMA response.

\begin{tabular}{|ccc|}
\hline Loading Condition & Applied Mass (g) & Applied Force (N) \\
\hline i & 697.5 & 6.842 \\
ii & 931.8 & 9.141 \\
iii & 1159.5 & 11.375 \\
iv & 1393.8 & 13.673 \\
\hline
\end{tabular}

Table 2.-Applied bias loads.

The results of this experiment revealed that as the load on the SMA wire increased, the temperatures at which it transformed also increased, along with the total

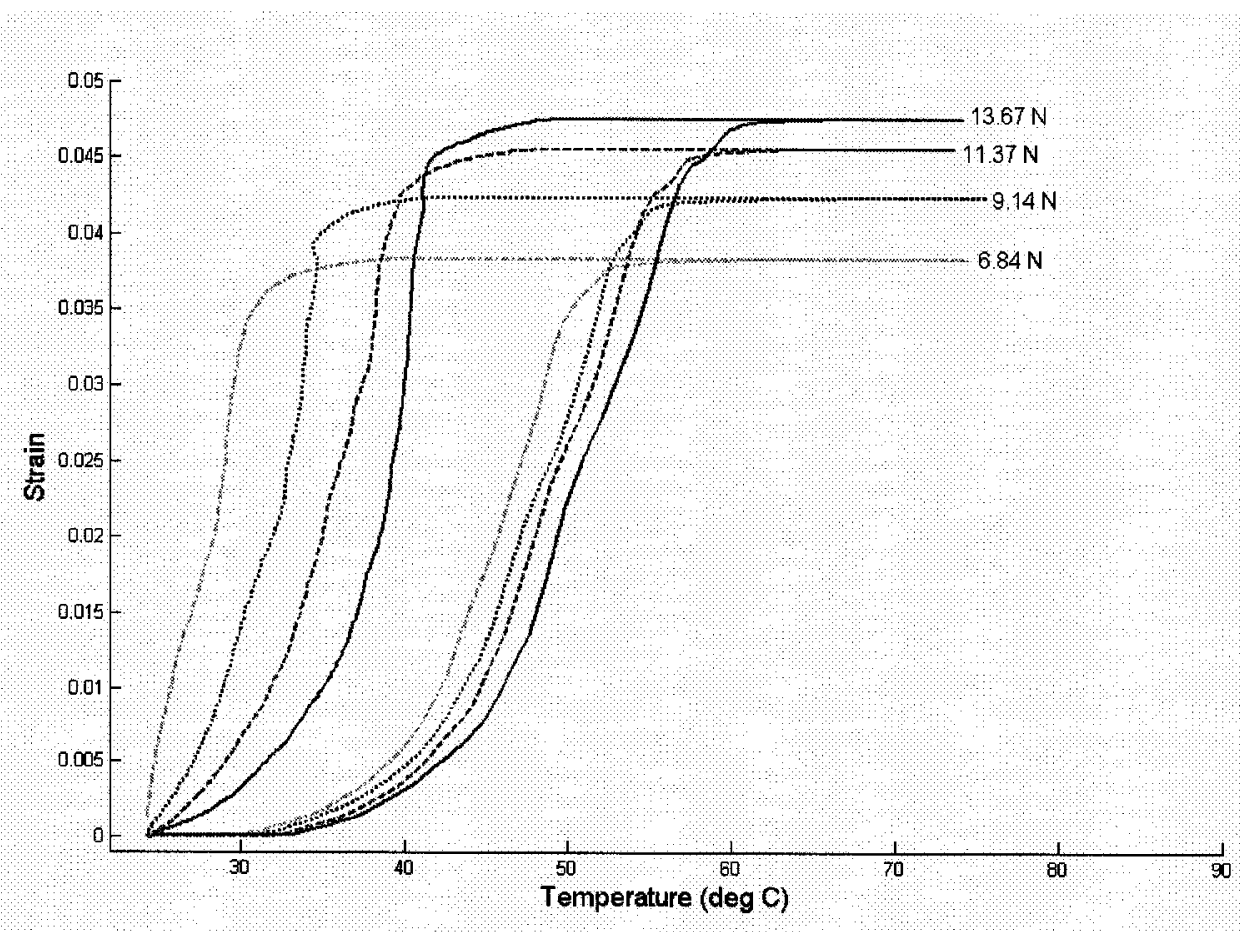

Figure 22.-Open loop responses to varying bias loads. 
actuation stroke. During these experiments, a large amount of friction between the shaft and bearings was observed as the shaft returned to its original position during cooling. This problem was alleviated by careful adjustment of the bolt connections between the apparatus' base and the bearing shoulders as well as maintaining proper lubrication.

Determination of the transformation temperatures from experimental data was conducted using Matlab. The hysteresis plot of shaft angle versus temperature revealed the temperatures at which the angle started and finished increasing and decreasing for both the heating and cooling portions of the actuation cycle. Two methods were employed for this process. The first method was inspired from previous work on shape memory alloys; linear portions of the hysteresis are extrapolated to form a quadrilateral, the vertices of which represent the transformation temperatures. Due to problems in modelling the transformation that will be discussed further, a second method was also adopted; the transformation temperatures were directly interpolated from the data at the locations where the shaft angle deviated from being constant.

\subsubsection{Material Characterization}

Modelling of the shape memory alloy behaviour required the estimation of various material properties. Some properties including the moduli of elasticity and coefficients of thermal expansion were assumed to be equal to values found in literature. Other properties, such as the transformation temperatures and stress influence coefficients were determined from data gathered during open-loop experiments.

Research performed on the validation of shape memory alloy models used the same actuator type (Flexinol). Values for the Young's moduli, coefficients of thermal 


\begin{tabular}{|ccc|}
\hline Property & Symbol & Value \\
\hline Young's Modulus (Austenite) & $E_{A}$ & $69.6 \mathrm{GPa}$ \\
Young's Modulus (Martensite) & $E_{M}$ & $33.1 \mathrm{GPa}$ \\
Coefficient of Thermal Expansion (Austenite) & $\alpha_{A}$ & $1.1\left(10^{-6}{ }^{\circ} \mathrm{C}^{-1}\right)$ \\
Coefficient of Thermal Expansion (Martensite) & $\alpha_{M}$ & $6.6\left(10^{-6}{ }^{\circ} \mathrm{C}^{-1}\right)$ \\
Maximum Residual Strain & $\varepsilon_{L}$ & 0.058 \\
\hline
\end{tabular}

Table 3.-Flexinol material properties assumed from literature [21].

expansion, and maximum residual strain were then assumed from this research [21], as shown in table 3.

It was observed that the Liang \& Rogers [22] shape memory alloy model depends upon the use of transformation temperatures that represent the absolute limits of the transformation. This is due to the use of the cosine function for representing the shape of the transformation curve. Considering equation 14 from the Liang \& Rogers model and neglecting the effects of stress, it is clear that the term within the cosine function will be equal to zero when $T=A_{s}$ and $\pi$ when $T=A_{f}$.

$$
\xi=\left(\frac{\xi_{0}}{2}\right)\left\{\cos \left(\pi \frac{T-A_{s}}{A_{f}-A_{s}}\right)+1\right\}, \quad A_{s}<T<A_{f}
$$

Eq. 14

The transformation curve is therefore defined as starting at $T=A_{s}$ and finishing at $T=A_{f}$ according to the model. However, typically the linear portion of the transformation curve is extrapolated to determine the transformation temperatures from experimental 


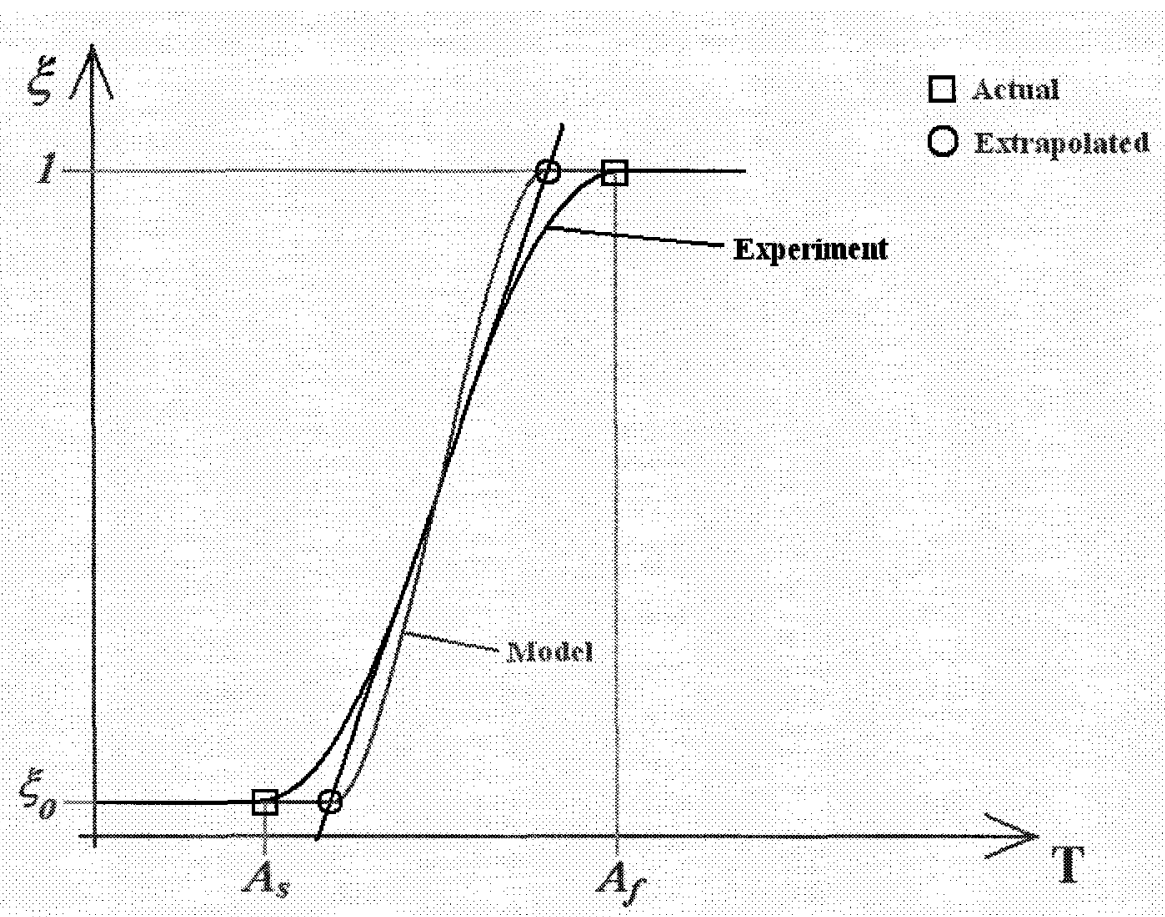

Figure 23.-Deviation between experimental data and model due to transformation temperature extrapolation.

data. This results in an inconsistency if such a method is used to extract the transformation temperatures and apply them within the Liang \& Rogers model. Figure 23 demonstrates a comparison between a typical hysteresis plot determined from experiment and the resulting model prediction applying the linearly extrapolated transformation temperatures - the actual and extrapolated transformation temperatures are encircled.

In order to account for this inconsistency, transformation temperatures were determined from inspection by simply observing a number of hysteresis plots and averaging the resulting data. Data from open-loop experiments was used to plot the transformation hystereses at various levels of applied stress. From these plots the actual transformation temperatures were determined (as defined in figure 23). This process was performed a number of times and the values averaged. A plot of transformation 
temperatures and applied stress revealed a linear relationship. The stress influence coefficients were determined from the slope, and zero-stress transformation temperatures from extrapolation, as shown in figures 24 and 25 . Table 4 summarizes the material properties determined from experimental data.

\begin{tabular}{|cccc|}
\hline & Property & Symbol & Value \\
\hline \multirow{3}{*}{ Transformation } & Austenite Start & $A_{s}$ & $34.19^{\circ} \mathrm{C}$ \\
Temperatures & Austenite Finish & $A_{f}$ & $44.00^{\circ} \mathrm{C}$ \\
& Martensite Start & $M_{s}$ & $20.76^{\circ} \mathrm{C}$ \\
Stress Influence & Martensite Finish & $M_{f}$ & $13.70{ }^{\circ} \mathrm{C}$ \\
Coefficients & Austenite & $C_{A}$ & $10.6 \mathrm{MPa} /{ }^{\circ} \mathrm{C}$ \\
& Martensite & $C_{M}$ & $5.9 \mathrm{MPa} /{ }^{\circ} \mathrm{C}$ \\
\hline
\end{tabular}

Table 4.-Flexinol material properties determined from experiment.

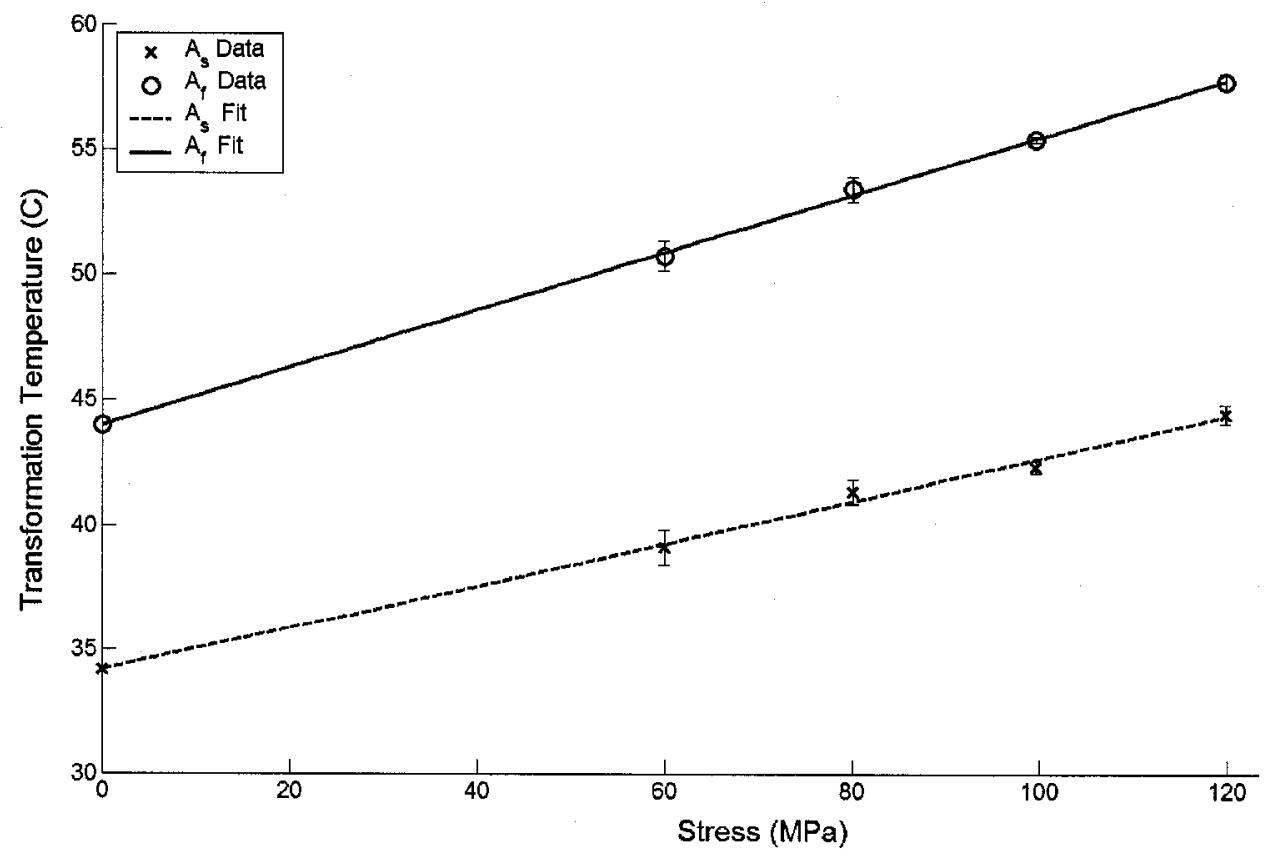

Figure 24.-Austenitic transformation temperatures determined from experiment and extrapolated. 


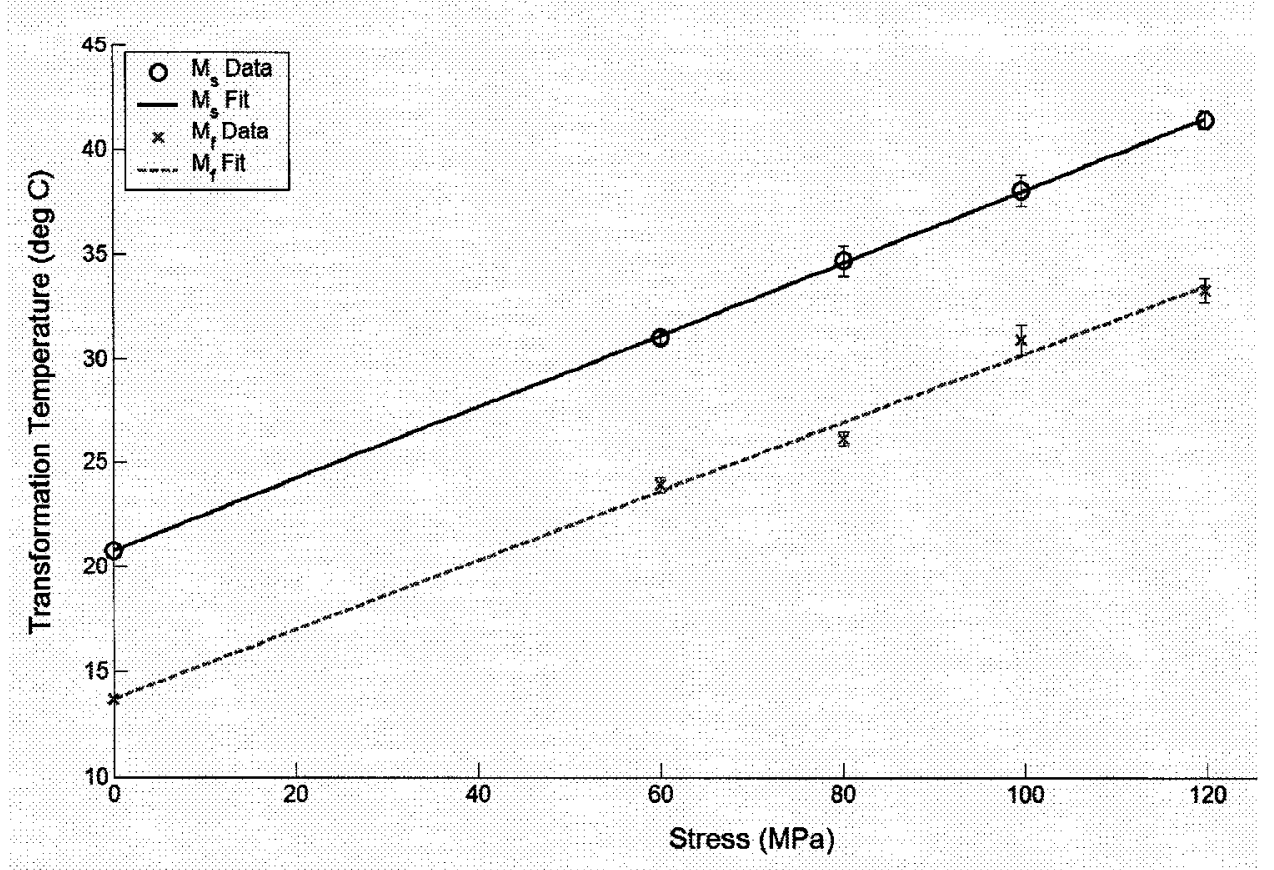

Figure 25.-Martensitic transformation temperatures determined from experiment and extrapolation.

\subsubsection{Single Channel Control Strategies}

The first set of control algorithms were developed using a single SMA actuator wire acting upon the shaft. The actuator provided a moment in one direction, while the bias load provided a moment in the opposite direction. The purpose of these experiments was to investigate the abilities of a single SMA actuator to maintain position control of the shaft angle. Various control algorithms were developed and improved upon to demonstrate the capabilities of the actuator wire.

Closed-loop control was implemented through LabView: user input specified a target shaft angle, and the LabView program attempted to maintain the shaft at that angle. 
The angular error signal, $\Delta$, was simply the difference between the actual shaft angle, $\theta$, and the target shaft angle, $\tau$, as used in a proportional control law.

$$
\Delta=\theta-\tau
$$

Eq. 15

Current through the SMA wire was then controlled based upon this error signal. Rotation in one direction could be accomplished using appropriate amounts of control current. However, rotation in the opposite direction required an absence of currentallowing the bias load to strain the wire. After observing the behaviour of the SMA wire to different amounts of current, control algorithm I was created using a look-up table. The assigned control currents based on the angular error signal are shown in Table 5. The desired tolerances of the angular error were set at \pm 0.05 degrees.

\begin{tabular}{|cc|}
\hline Angular Error $\left(^{\circ}\right)$ & Current (A) \\
\hline $180 \geq \Delta>0.05$ & 0.85 \\
$0.05 \geq \Delta \geq 0$ & 0.75 \\
$0>\Delta \geq-0.05$ & 0.60 \\
$-0.05>\Delta \geq-180$ & 0.40 \\
\hline
\end{tabular}

Table 5.-Look-up table of applied current values for control algorithm $I$.

It was found that a current of $0.85 \mathrm{~A}$ provided sufficient actuation moment to induce a rotation of the shaft in the 'upwards' direction. Shaft rotation in the 'downwards' direction was accomplished using 0.40 A, which allowed the SMA wire to 


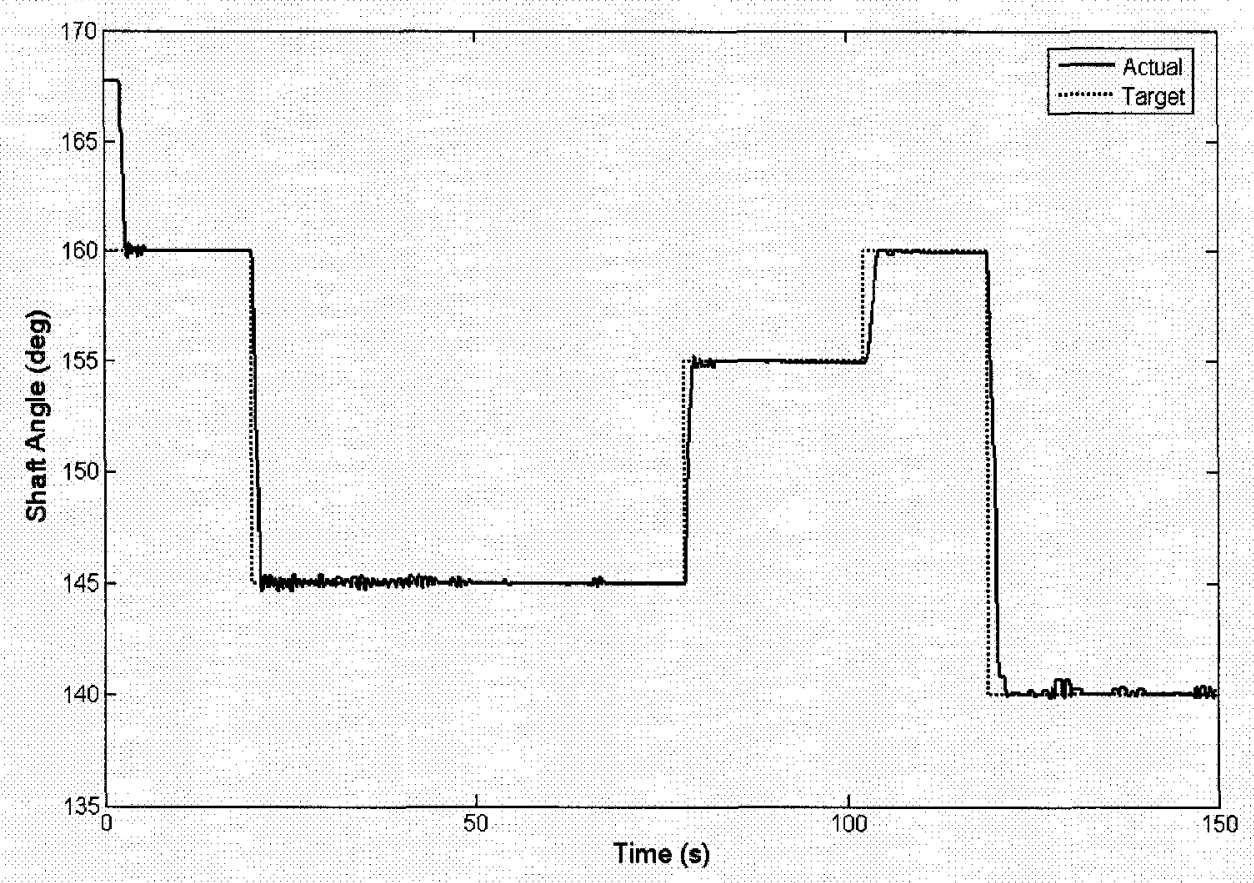

Figure 26.-Shaft response to varying target angles using control algorithm $I$.

cool enough that the bias load overcame the actuation moment. It should be noted that the use of no current to induce a rotation in the 'downward' direction was undesirable since thermal inertia effects caused some instability. The applied control current when the shaft angle was within the error bandwidth was adjusted to provide similar currents but at slightly modified levels in order to induce smaller corrections. It was found that currents of $0.75 \mathrm{~A}$ and $0.60 \mathrm{~A}$ were sufficient to maintain relatively constant temperature and hence maintain the shaft angle to within the error tolerance bandwidth while also correcting slight error deviations as seen in figure 26.

The success of this control algorithm depended upon the static nature of the bias load. It was recognized that changes in the bias load would require changes in the constant current levels shown in table 5, due to the dependence of the SMA 
transformation temperatures upon the applied stress. A new control algorithm was created to increment the control current therefore adapting to changes in the bias load. The compound error signal, $\varepsilon$, was developed to account for the position error, $\Delta$, and velocity, $\omega$, of the shaft angle similar to a proportional-derivative control with zero target velocity. Due to the intensification of noise from differentiation, the angular velocity signal was adjusted to include a deadband within $\pm 0.005 \mathrm{deg} / \mathrm{s}$ of the zero velocity point.

$$
\varepsilon=\Delta+\omega
$$

Equation 4 shows the relationship between the error signal, angular position error, and angular velocity of the shaft-similar to a proportional-derivative control law. If the direction of shaft motion is opposite to the direction of angular error, the error signal is low; if the direction of shaft motion is in the direction of the angular error, the error signal is high. The applied control current was then arbitrarily increased or decreased by $0.01 \mathrm{~A}$ based upon the sign of the error signal, as shown in table 6.

\begin{tabular}{|cc|}
\hline Error Signal & Change in Applied Current (A) \\
\hline$\varepsilon \geq 0$ & +0.01 \\
$\varepsilon<0$ & -0.01 \\
\hline
\end{tabular}

Table 6.-Applied current logic for control algorithm II.

This control strategy proved successful for varying bias loads. However, it also resulted in a slower response and high overshoot due to the small change in applied 


\begin{tabular}{|ccc|}
\hline Angular Error & Angular Velocity & Change in Applied Current (A) \\
\hline$\Delta \geq 0$ & $\omega \geq 0$ & +0.1 \\
$\Delta \geq 0$ & $\omega<0$ & 0 \\
$\Delta<0$ & $\omega>0$ & 0 \\
$\Delta<0$ & $\omega \leq 0$ & -0.1 \\
\hline
\end{tabular}

Table 7.-Modified applied current logic for control algorithm II.

current. In order to improve this response, the increment in applied current was increased. The control strategy was also slightly modified. Instead of relying on the sign of the compound error signal, $\varepsilon$, the control algorithm used a set of logical conditions as shown in table 7.

The set of logical conditions leads to the following strategy: if the shaft angle is

\begin{tabular}{|ccc|}
\hline Angular Error $\left(^{\circ}\right)$ & Angular Velocity & Change in Applied Current (A) \\
\hline$\Delta>7.5$ & $\omega<0$ & -0.01 \\
& $\omega \geq 0$ & +0.05 \\
$7.5 \geq \Delta>0.05$ & $\omega<0$ & -0.025 \\
& $\omega \geq 0$ & +0.01 \\
$0.05 \geq \Delta>0$ & $\omega \leq 0$ & 0 \\
$0 \geq \Delta>-0.5$ & $\omega>0$ & +0.01 \\
& $\omega \leq 0$ & -0.005 \\
$-0.5 \geq \Delta>-1$ & $\omega>0$ & +0.001 \\
& $\omega \leq 0$ & -0.01 \\
$-1 \geq \Delta$ & $\omega>0$ & +0.001 \\
& $\omega \leq 0$ & -0.1 \\
& $\omega>0$ & +0.01 \\
\hline
\end{tabular}

Table. 8-Conditions for control algorithm III. 
too high and is not decreasing, the applied current is increased; if the shaft angle is too low and is not increasing, the applied current is decreased; otherwise, the applied current is left unchanged. Larger increments in current decreased the response time, but also resulted in some instability. It was also observed that less control effort was required when allowing the bias load to actuate the shaft. Therefore, casual control algorithm III was developed to increment the applied current using a much more complex set of logical conditions.

The range of the shaft angular position error was divided into a set of regions, each of which was given a unique pair of current increment values dependent upon the shaft's direction of motion. This combined the success of the look-up table algorithms and adaptability of current increments. Table 8 summarizes the current increment values

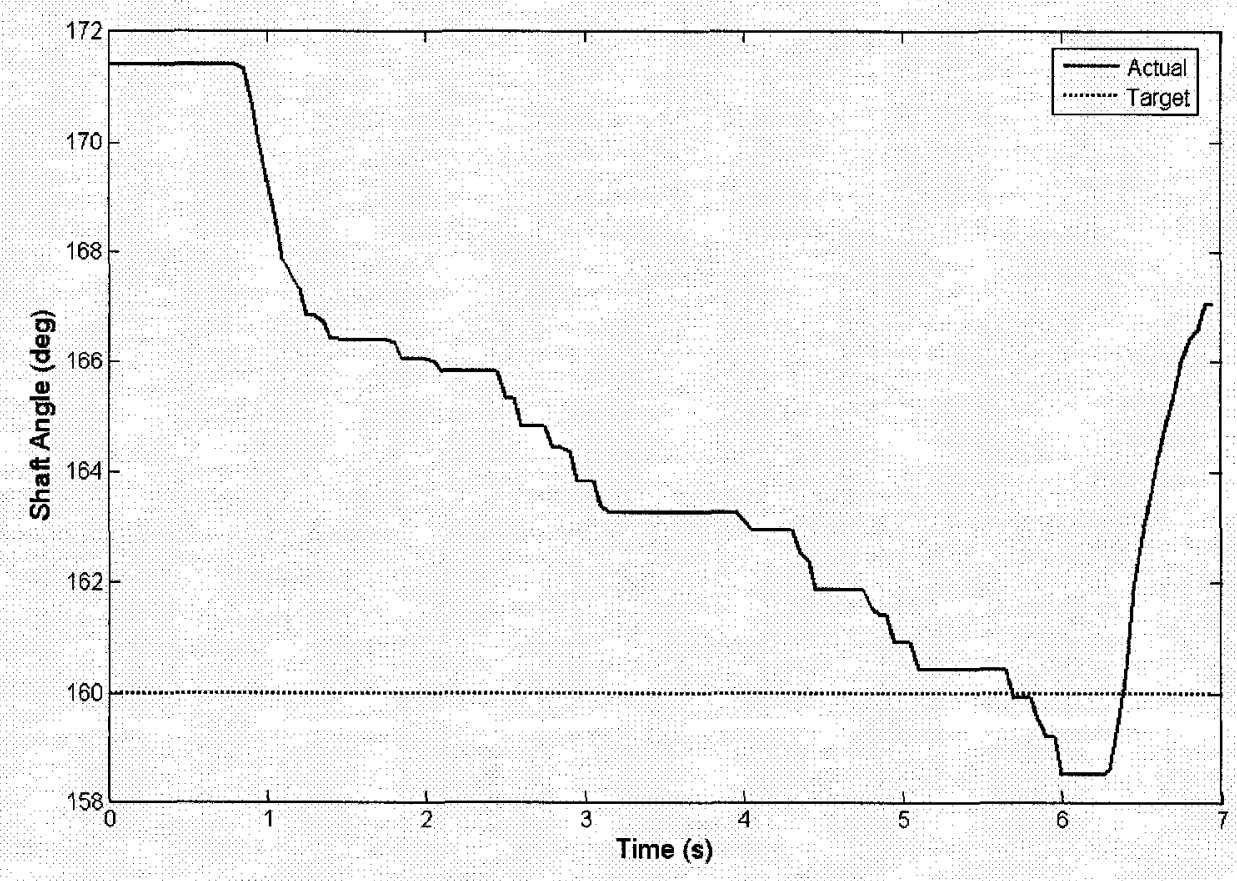

Figure 27.-Shaft response to varying target angle using control algorithm III. 
associated with each region.

As seen in figure 27, control algorithm III did not improve the response. It was found that the use of increments instead of directly changing the applied current value resulted in slow response times and a phase lag between the control effort and shaft response. The use of both direct changes as well as incremental changes in applied current was implemented in control algorithm IV.

The applied current was initialized at a reference value that remained constant throughout the experiment, $I_{\text {ref. }}$. This reference value was then summed with an offset value that was initialized at zero and incremented according to the controller, $I_{\text {off. }}$. Finally, the sum of these values, $I_{0}$, was multiplied by a gain, $\gamma$, determined by the shaft angular error. Table 9 summarizes the current increments and gains associated with each angular error region.

The sign of the incremental change in current depended upon the aforementioned

\begin{tabular}{|ccc|}
\hline Angular Error $\left(^{\circ}\right)$ & Change in Applied Current (A), $\delta \mathrm{I}$ & Gain, $\gamma$ \\
\hline$\Delta>1$ & 0.025 & 1 \\
$1 \geq \Delta>0.1$ & 0.01 & 0.8 \\
$0.1 \geq \Delta>0$ & 0.001 & 0.7 \\
$0 \geq \Delta>-0.1$ & 0.001 & 0.6 \\
$-0.1 \geq \Delta>-1$ & 0.01 & 0.5 \\
$-1 \geq \Delta$ & 0.025 & 0.4 \\
\hline
\end{tabular}

Table 9.-Current increments and regional gains for control algorithm IV. 
compound error signal, $\varepsilon$. A deadband was introduced into the compound error signal between \pm 0.1 within which the shaft was considered to have reached its target. If the compound error signal was greater than 0.1 , the current was decreased; if the compound error signal was less than -0.1 , the current was increased.

$$
\left(I_{o f f}\right)_{n}=\left(I_{o f f}\right)_{n-1} \pm \delta I
$$

$$
I_{0}=I_{r e f}+I_{o f f}
$$

$$
I_{\text {out }}=\mathscr{H}_{0}
$$

Control algorithm IV proved to be the most successful for a single SMA actuator wire acting against a bias load. The use of a look-up table for increments in current combined with a second look-up table for applied current gains led to both quick responses as well as bias load adaptability. The controller was able to track the target angle with little overshoot and a satisfactory response time as well as maintain the target angle during changes in bias load. Figure 28 demonstrates the response of the shaft angle to varying target angles using this control algorithm. 


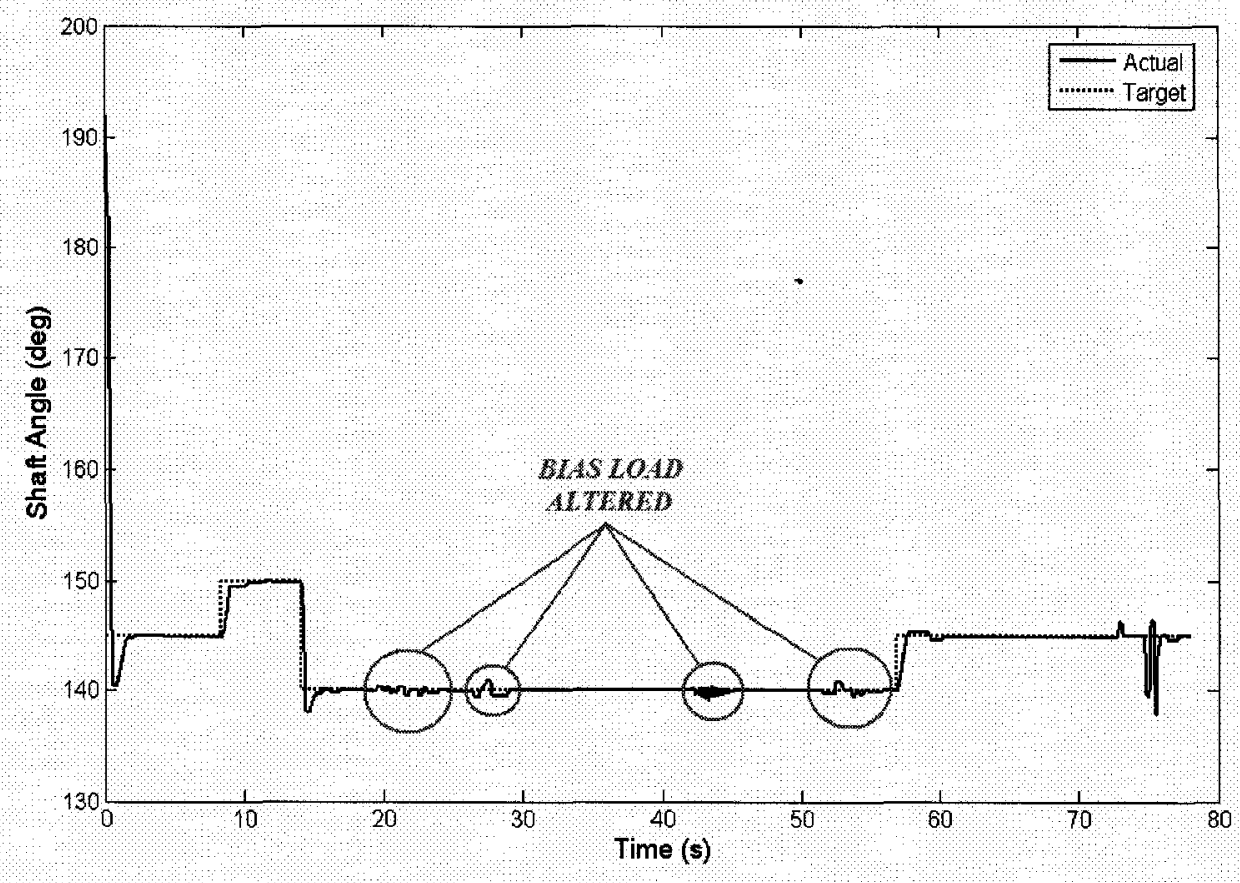

Figure 28.-Shaft response to varying target angle using control algorithm IV.

\subsubsection{Dual Channel Control Strategies}

Control of the shaft angular position was investigated using two SMA actuator wires mounted antagonistically. The use of two wires allowed controlled actuation of the shaft in both clockwise and counter-clockwise directions. Due to the connection of the SMA wires directly to the steel shaft, the conduction path of the two wires interfered with one another. Therefore, pulley blocks - nylon pulleys pinned within aluminium caseswere introduced into the apparatus in order to isolate the current in the SMA wires. Screw terminals constrained each end of the SMA wires and the pulley blocks were linked to the shaft with turnbuckles and steel bands. The turnbuckles allowed for adjustment of the preloaded tension within the SMA wires. 


\begin{tabular}{|cccc|}
\hline \multirow{2}{*}{ Angular Position Error $\left(^{(}\right)$} & Angular Velocity & \multicolumn{2}{c|}{ Applied Current (A) } \\
& $\omega<0$ & Ch.1 & Ch.2 \\
\hline \multirow{2}{*}{$\Delta>0.5$} & $\omega \geq 0$ & 0.8 & 0 \\
$0.5 \leq \Delta<0$ & $\omega<0$ & 1.0 & 0 \\
& $\omega \geq 0$ & 0 & 0 \\
$0 \leq \Delta<0.5$ & $\omega<0$ & 1.0 & 0 \\
& $\omega \geq 0$ & 0 & 1.0 \\
$\Delta \leq 0.5$ & $\omega<0$ & 0 & 0 \\
& $\omega \geq 0$ & 0 & 1.0 \\
\hline
\end{tabular}

Table 10.-Conditions for control algorithm $V$.

Building upon experience gained controlling the shaft using a single SMA actuator, the first dual channel control algorithm, control algorithm V, implemented a similar set of logical conditions shown in table 10 . The angular position error was divided into regions and constant values of current were assigned for both channels, depending upon the direction of shaft motion.

Although the controller was able to maintain a target angle, small perturbations upon the shaft led to some instability. It was found that the method used by LabView to output to the data acquisition system caused the applied current to maintain its value until updated by the next iteration of the controller. The control algorithm was therefore altered to output square wave pulses instead of constant current updates. Using a duty cycle of $50 \%$, amplitudes of some of the current pulses were also increased to maintain a similar total energy input, while others were decreased or left unchanged in order to input 


\begin{tabular}{|cccc|}
\hline \multirow{2}{*}{ Angular Position Error $\left(^{\circ}\right)$} & Angular Velocity & \multicolumn{2}{c|}{ Applied Current (A) } \\
& $\omega<0$ & Ch.1 & Ch.2 \\
\hline$\Delta>0.5$ & $\omega \geq 0$ & 0.75 & 0 \\
$0.5 \leq \Delta<0$ & $\omega<0$ & 1.5 & 0 \\
& $\omega \geq 0$ & 0 & 0 \\
$0 \leq \Delta<0.5$ & $\omega<0$ & 1.0 & 0 \\
& $\omega \geq 0$ & 0 & 1.0 \\
$\Delta \leq 0.5$ & $\omega<0$ & 0 & 0 \\
& $\omega \geq 0$ & 0 & 1.5 \\
& & 0 & 0.75 \\
\hline
\end{tabular}

Table 11.-Modified conditions and current values for dual channel control algorithm $V$.

less energy. Table 11 summarizes the altered current values for the logical conditions previously presented.

Figure 29 shows the shaft response to random manual excitation. Solid lines represent the response with control applied, while dotted lines represent control off. The use of the compound error signal was also investigated for the dual channel arrangement. However, the compound error signal was redefined by equation 20 .

By setting the weight of the angular error to be twice that of the angular velocity, the new signal represented zero error when the shaft was projected to reach its target in $0.5 \mathrm{~s}$. The new compound error signal was introduced into control algorithm VI as shown in table 12.

$$
\varepsilon=2(\theta-\tau)+\omega
$$

Eq. 20 


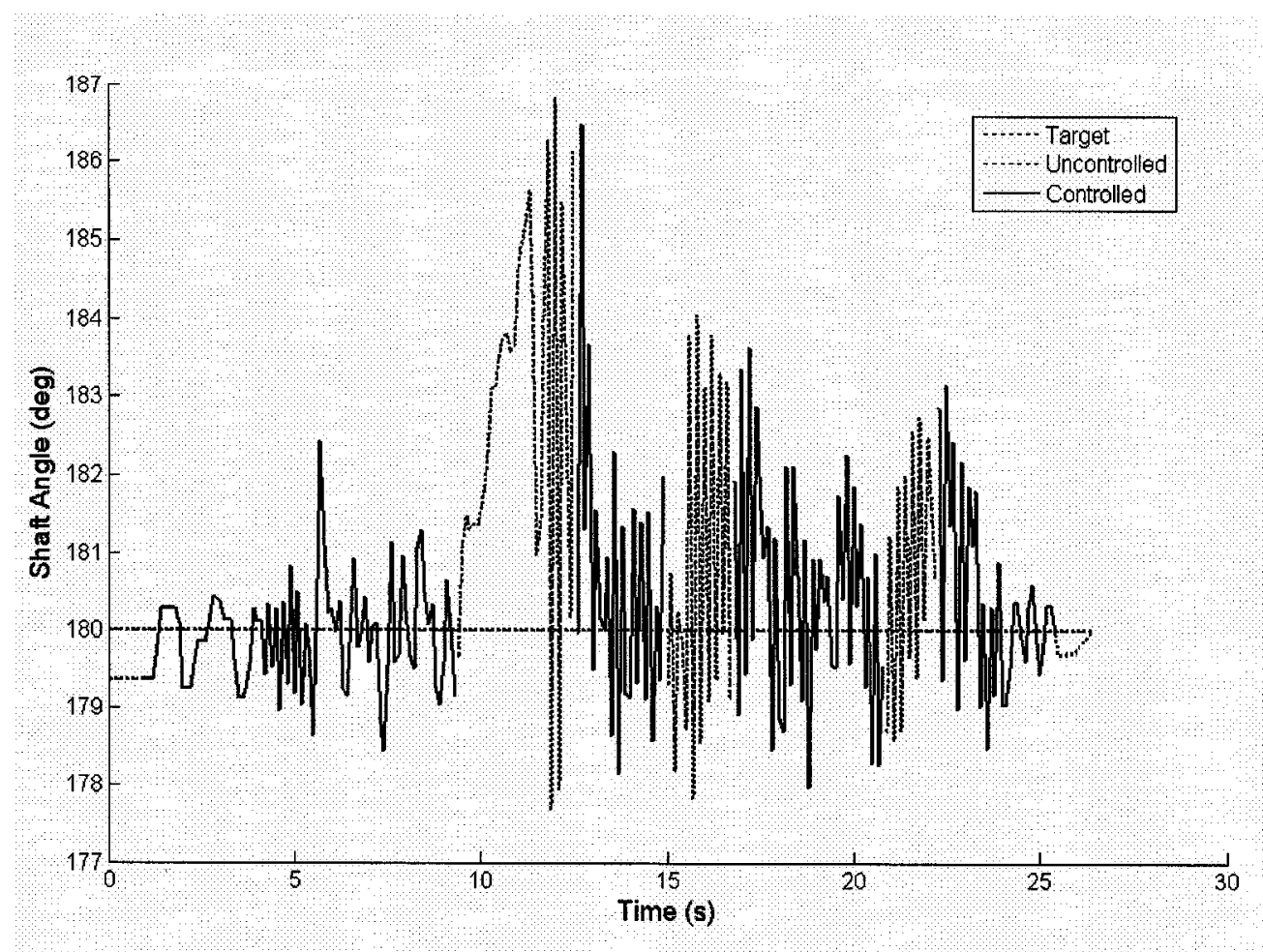

Figure 29.-Shaft response to random excitation using control algorithm $V$.

For this control algorithm, actuation was accomplished using a square wave pulse, with a $60 \%$ duty cycle and a magnitude of $1.2 \mathrm{~A}$. Data was sampled at $10000 \mathrm{~Hz}$ and averaged over 250 samples, leading to an effective sampling and control rate of $40 \mathrm{~Hz}$.

Figure 30 shows the shaft response to random excitation using control algorithm

\begin{tabular}{|cc|}
\hline Error Signal & Action \\
\hline$\varepsilon>0.1$ & Actuate Top \\
$\varepsilon<-0.1$ & Actuate Bottom \\
$-0.1 \leq \varepsilon \leq 0.1$ & No Actuation \\
\hline
\end{tabular}

Table 12.-Conditions for control algorithm VI. 


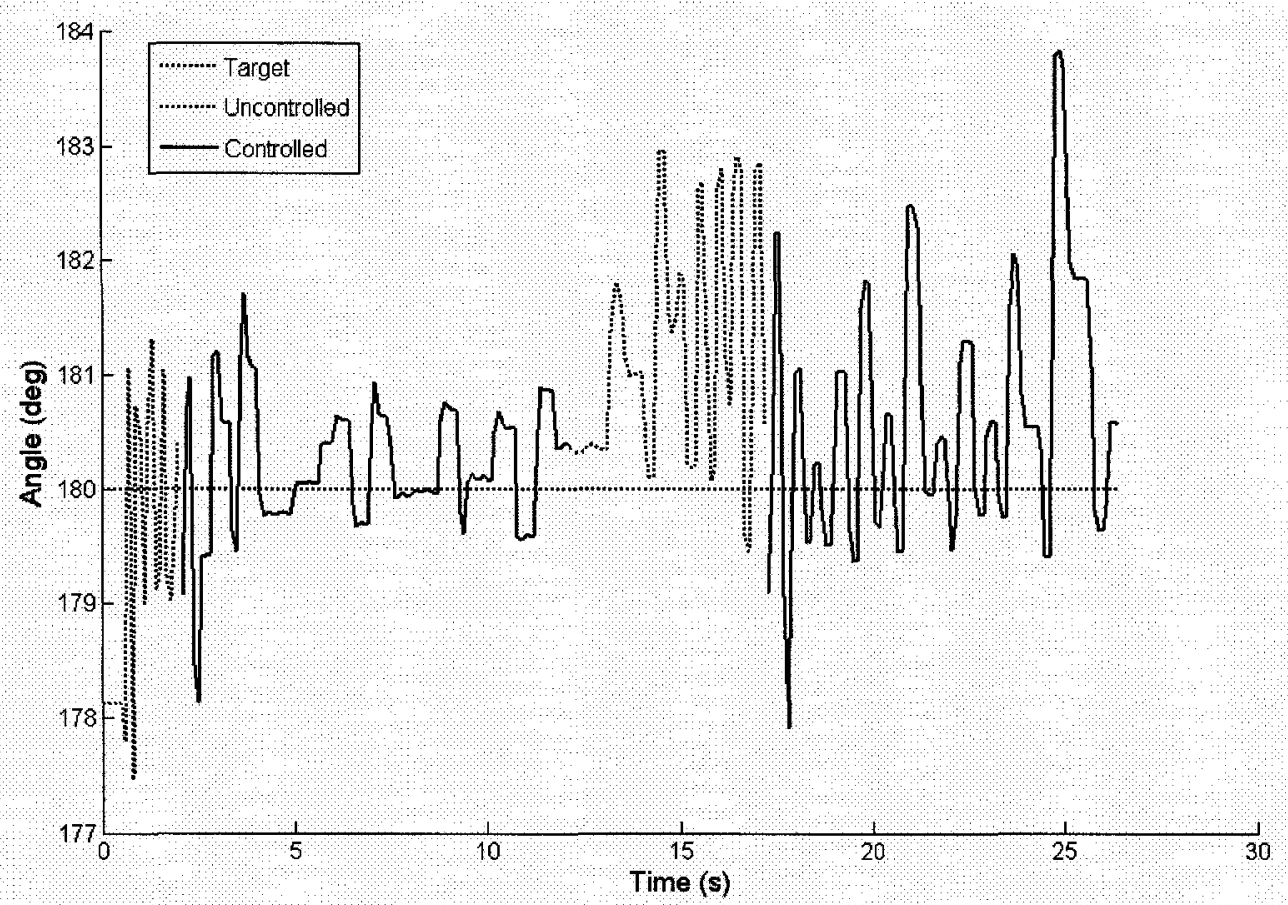

Figure 30.-Shaft response to random excitation using modified control algorithm VI.

VI. The amplitude of the current pulse proved to be too small to actuate the SMA wire adequately if the error was very large. Conversely, larger amplitudes would cause instability and high overshoot if the error was small. Therefore, control algorithm VI was modified to set the current magnitude proportional to the error signal, as shown in equation 21.

$$
I_{\text {out }}=3|\mathcal{E}|
$$

Eq. 21

The use of proportional current amplitude improved the shaft response greatly. However, the linear relationship led to instability when the error signal was very large. 
This caused a large amount of current to be sent to the SMA wire leading to saturation of the actuator and either divergence or oscillations as seen in figure 30 . The solution to this problem was simply to alter the relationship from being linear to an exponential relationship. Assuming an exponential of 0.25 as shown in equation 22 , the new relationship was implemented into control algorithm VII. The current input signal was reverted from a square wave pulse back to an instantaneous value update. Also, the compound error signal was reverted back to equation 16 , with no additional weight placed upon the angular error term.

$$
I_{\text {out }}=|\varepsilon|^{1 / 4}
$$

Eq. 22

This relationship between error signal and applied current was implemented using similar logical conditions as table 12 , but with a smaller angular error bandwidth as shown in table 13.

\begin{tabular}{|cc|}
\hline Error Signal & Action \\
\hline$\varepsilon>0.05$ & Actuate Top \\
$\varepsilon<-0.05$ & Actuate Bottom \\
$-0.05 \leq \varepsilon \leq 0.05$ & No Actuation \\
\hline
\end{tabular}

Table 13.-Modified conditions for control algorithm VII.

The response was greatly improved by the use of an exponential relationship between error signal and applied current as shown in figure 31. It was found that high 


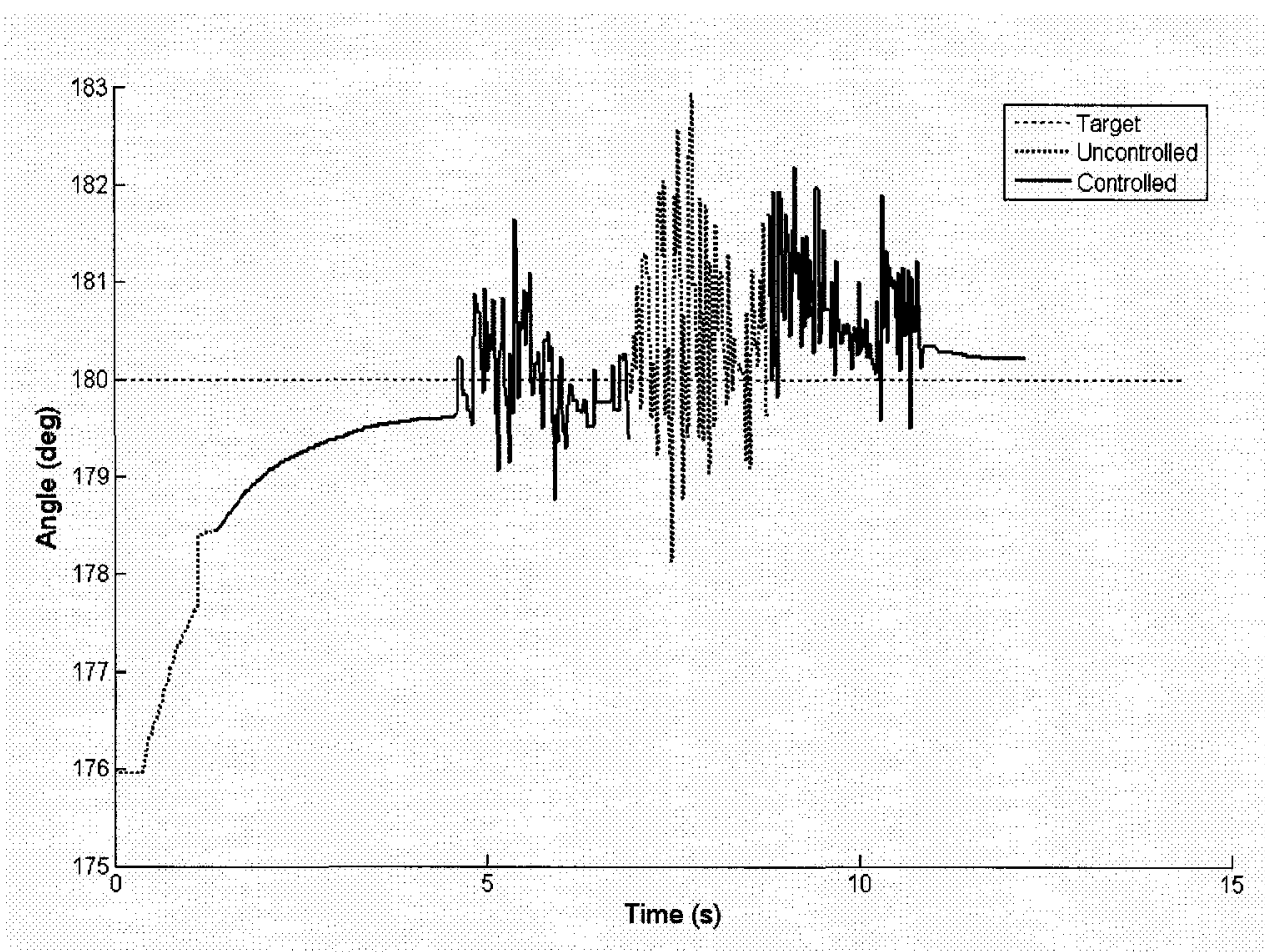

Figure 31.-Shaft response to random excitation using control algorithm VII.

angular velocities still led to some instability, and so it was desirable to reinforce the control algorithm's dependence upon the angular error term. However, instead of redefining the compound error signal, another exponential term-dependent upon the angular error alone-was added to the applied current formula. Equation 23 represents the altered formula for the applied current used in control algorithm VIII.

$$
I_{\text {out }}=\operatorname{sgn}(\Delta)\left(|\Delta|^{1 / 4}\right)+\operatorname{sgn}(\varepsilon)\left(\frac{|\varepsilon|^{1 / 4}}{5}\right)
$$

Eq. 23

The altered formula associated position errors with a greater weight than velocity errors, especially when the angular error was high. This imposed more restriction upon 


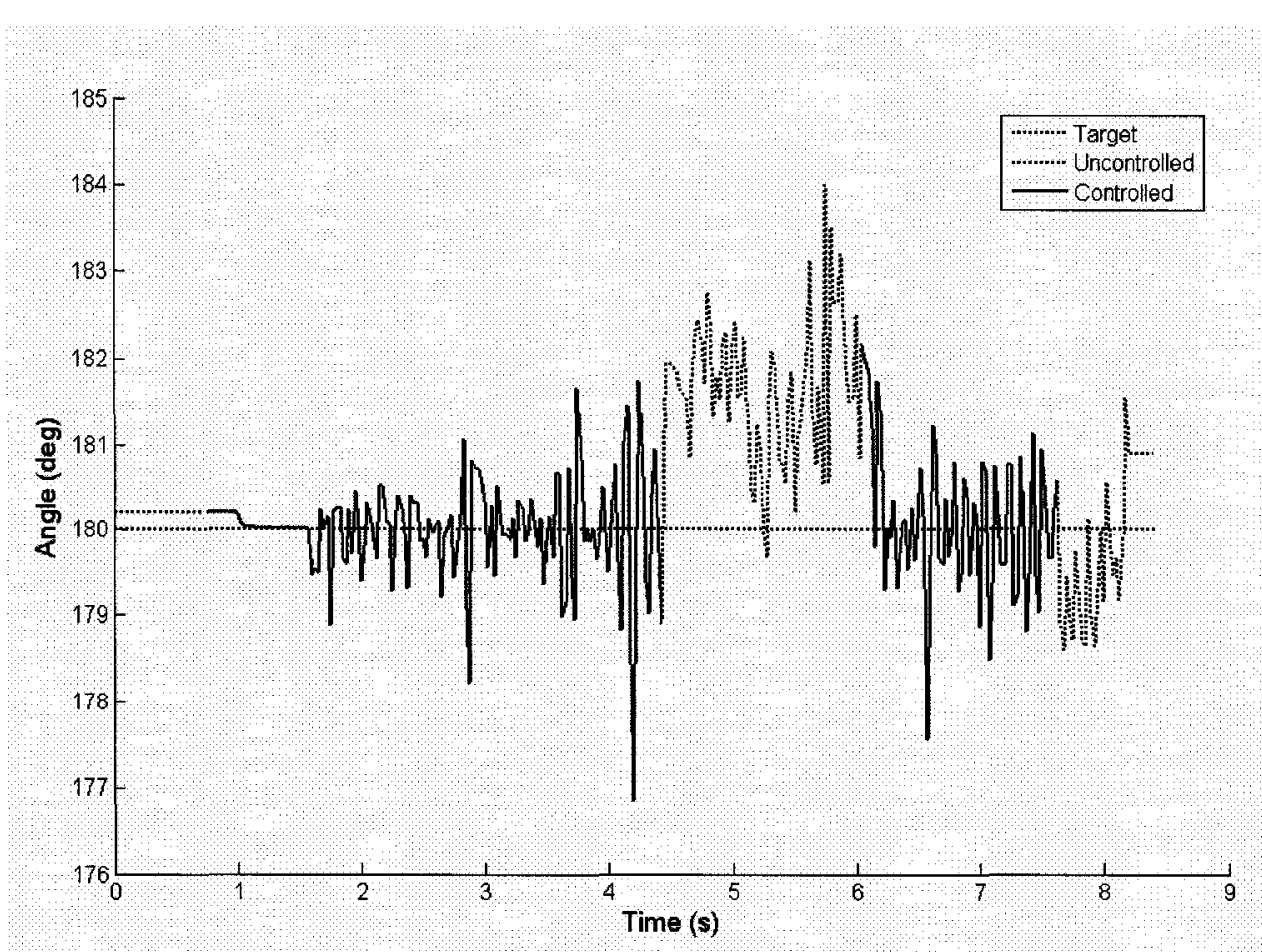

Figure 32.-Shaft response to random excitation using control algorithm VIII.

the angular velocity of the shaft, increasing the damping effect and creating greater stability. The choice of which actuator to activate was determined by the overall sign of the applied current value. Figure 32 shows the shaft response using control algoritm VIII.

It was desired to further increase the control algorithm's sensitivity to the direction of motion-imposing greater controller effort when the shaft angle was diverging and less controller effort when the shaft angle was converging. This was accomplished by multiplying the second term in equation 23 by a factor given by equation 24.

$$
\Psi=e^{\left(\frac{\Delta \omega}{1000}\right)}
$$


The use of the exponential term caused the value of $\Psi$ to be very small if both the angular error and angular velocity were opposite in sign, and very large if they were similar in sign. The divisor was chosen by trial and error to appropriately scale the overall value of $\Psi$ and avoid saturating the applied current- $\Psi$ typically remained less than a value of 2. The overall formula for control algorithm IX is then given by equation 25 . Figure 33 illustrates the applied current as a function of shaft error and velocity.

$$
I_{\text {out }}=\operatorname{sgn}(\Delta)\left(\frac{|\Delta|^{\alpha}}{\beta}\right)+\operatorname{sgn}(\varepsilon)\left(\frac{e^{\left(\frac{\Delta \omega}{1000}\right)}|\varepsilon|^{\gamma}}{\delta}\right)
$$

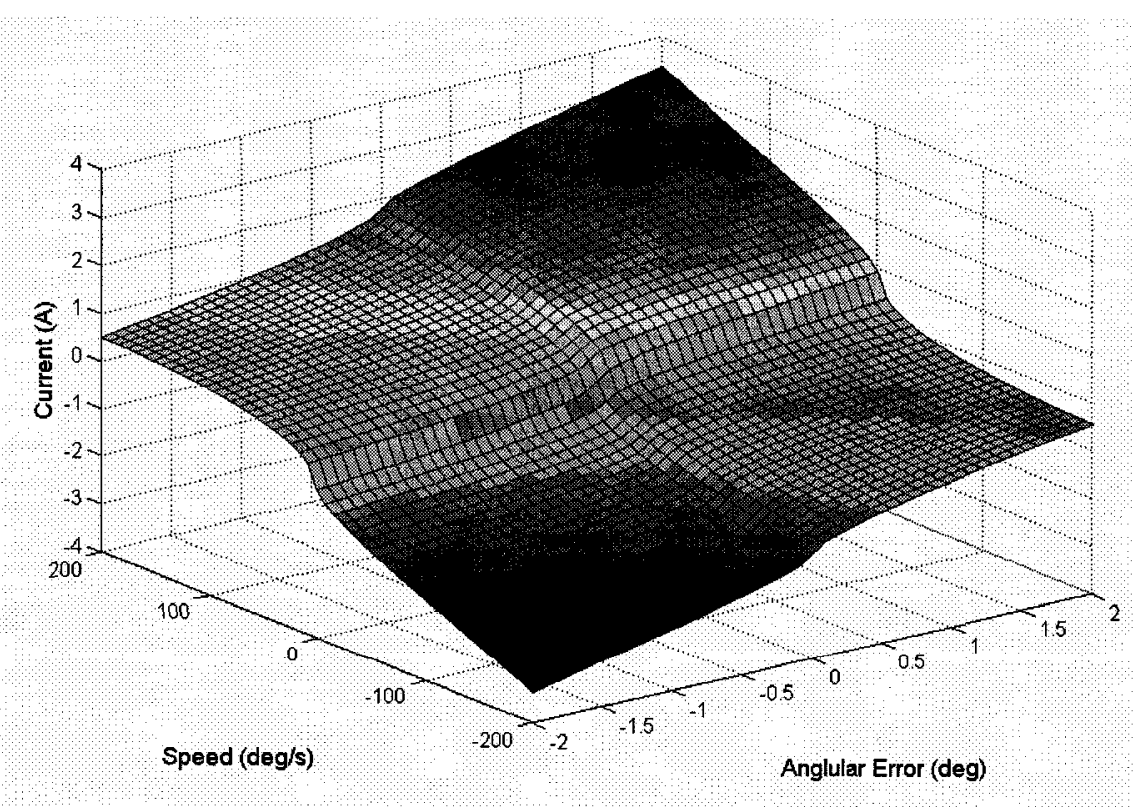

Figure 33.-Visualization of current output for control algorithm IX. Positive and negative current values correspond to the top and bottom actuator channels respectively. 


\begin{tabular}{|c|cccc|}
\hline Coefficient & $\alpha$ & $\beta$ & $\gamma$ & $\delta$ \\
Value & 0.5 & 2 & 0.5 & 8 \\
\hline
\end{tabular}

Table 14.-Experimentally determined values for coefficients in equation 15.

The values of the coefficients within equation 13 were chosen through trial and error. Table 14 shows the values that were determined through experimentation and yielded the best response.

As seen in figure 34 , control algorithm IX proved to be the most successful for a dual channel controller. Varying the target angle led to a quick and stable response. Small perturbations and random manual excitation upon the shaft did not lead to instability as was found in previous control algorithms.

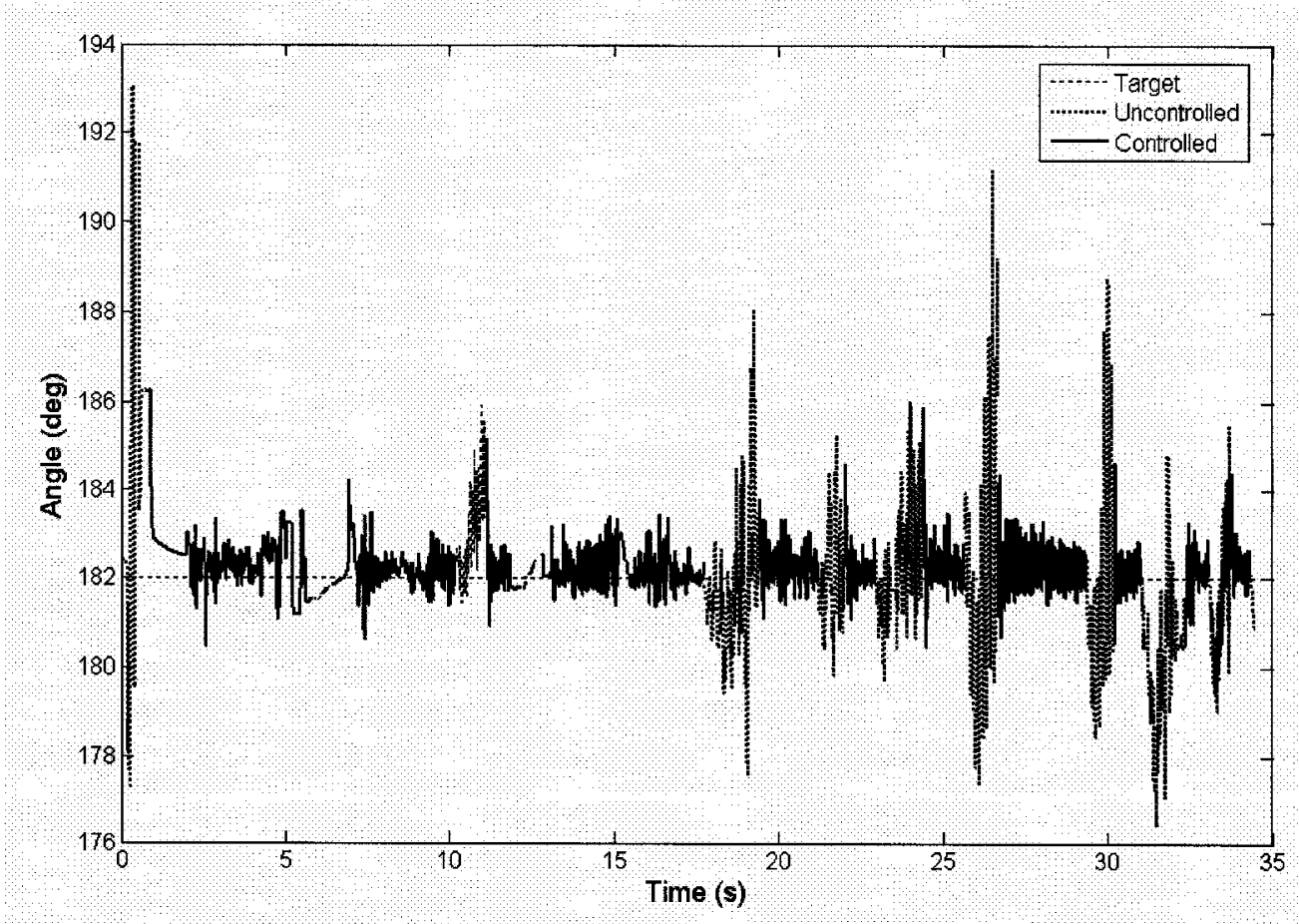

Figure 34.-Shaft response to random excitation using control algorithm VIII. 


\subsubsection{PID Control \& Tuning}

Although it was assumed that a linear-time-invariant solution for controlling the SMA wires would not be successful, attempts were made at classical P, PI, and PID controllers. The gains selected for the controller were based upon Ziegler-Nichols tuning methods.

The first Ziegler-Nichols tuning method required the system response to a step input. This was accomplished through a sudden change in target angle. The response was recorded and the 'dead time', $L$, and 'time constant', $T$, determined from the results. From the values of $L$ and $T$, appropriate gains for a P, PI, and PID controller were determined. Table 15 summarizes the results of the first Ziegler-Nichols tuning method.

\begin{tabular}{|cccc|}
\hline \multirow{2}{*}{ Gain } & \multicolumn{3}{c|}{ Controller } \\
& $\mathrm{P}$ & $\mathrm{PI}$ & $\mathrm{PID}$ \\
\hline$K_{p}$ & 0.8644 & 0.7780 & 1.0373 \\
$T_{i}$ & $\infty$ & 0.0885 & 0.0590 \\
$T_{d}$ & 0 & 0 & 0.0147 \\
\hline
\end{tabular}

Table 15.-Gains determined through Ziegler-Nichols tuning method I.

The gains were implemented into the control algorithm using a PID block within LabView. The block depended upon the angular position error signal only and internally evaluated the derivative and integral of this signal. The performance of the P and PI controllers was satisfactory for static loads and changing target angles, but showed instability during random excitation. The PID controller caused a large amount of 
overshoot and often diverged. This was assumed to be a result of the linear relationship within the PID controller, causing high amounts of applied current when the error was large and saturating the SMA wires.

The second Ziegler-Nichols tuning method required that a proportional controller be observed while increasing the gains until steady oscillations occured. Steady oscillations were achieved when the gain was increased to $K_{u}=2.4$. The response was then analyzed in Matlab using a Fourier transform. The spectrum of the Fourier transform showed oscillations occurring at a frequency of $19.53 \mathrm{~Hz}$, or a critical period of $P_{c r}=$ 0.0512 s. Ziegler-Nichols rules for the second tuning method were then implemented. From the ultimate gain, $K_{u}$, and critical period, $P_{c r}$, the required gains and time constants for P, PI, and PID control were calculated as shown in table 16.

\begin{tabular}{|cccc|}
\hline Gain & \multicolumn{3}{c|}{ Controller } \\
& $\mathrm{P}$ & $\mathrm{PI}$ & $\mathrm{PID}$ \\
\hline$K_{p}$ & 1.2 & 1.08 & 1.44 \\
$T_{i}$ & $\infty$ & 0.04267 & 0.0256 \\
$T_{d}$ & 0 & 0 & 0.0064 \\
\hline
\end{tabular}

Table 16.-Gains determined through Ziegler-Nichols tuning method II.

The shaft response to random excitations and varying target angles was similar to the results of the first Ziegler-Nichols tuning method. Both the P and PI controllers performed with little overshoot or divergence under static loads and small changes in target angle. However, instability prevailed when random excitation was introduced. The 
PID controller was generally unstable-likely due to the non-linearity of the system.

Although the system performance was not greatly improved by P, PI, or PID control, it was conceived that such controllers could be beneficial if a complex set of transfer functions were determined for governing the system behaviour. 


\section{Mathematical Models}

The transformation of the shape memory alloy wires was modeled using the Liang \& Rogers model previously described and applied to mathematical models of the single channel and dual channel configurations to determine the angular position of the shaft. Experimental data gathered during open loop responses was used to validate the mathematical models of the single channel and dual channel configurations.

\subsection{Single Channel System Model}

The first model incorporated only a single SMA wire actuating the shaft from the top. A constant load was imposed using the aforementioned pulley system. Since the system was statically determinate, the tension in the SMA wire was always assumed to be equal to the tension in the pulley cable. Therefore, neglecting inertia, the constitutive equation can be re-written as equation 26.

$$
\Delta \varepsilon=\varepsilon_{L} \Delta \xi-\alpha(\xi) \Delta T
$$

Eq. 26

The shaft angle was then related to the strain in the SMA wire by equation 27 , where $L$ is the length of the SMA wire, and $r$ is the radius of the shaft. 


$$
\Delta \theta=\Delta \varepsilon\left(\frac{L}{r}\right)
$$

Eq. 27

A Matlab program was then written to simulate the actuation of the SMA wire in this system. The time history of temperatures was given as an input, as well as the bias load on the pulley, and the initial martensite fraction. Stress remained a constant throughout the simulation. Therefore, the phase transformation equations and constitutive equation could be solved for martensite fraction and strain respectively.

\subsection{Single Channel Simulation Results}

The single channel model was simulated using properties for the system given in table 17. Stress was assumed to be uniform across the circular section of the SMA wire. Temperature data from experimental open loop responses was interpolated cubically to increase the resolution of the data.

\begin{tabular}{|cc|}
\hline Property & Value \\
\hline Shaft Radius, $r$ & $6.35 \mathrm{~mm}$ \\
SMA Wire Length, $L$ & $181 \mathrm{~mm}$ \\
SMA Wire Diameter & $0.381 \mathrm{~mm}$ \\
Applied Bias Load & $13.67 \mathrm{~N}$ \\
\hline
\end{tabular}

Table 17.-Single channel model simulation parameters. 


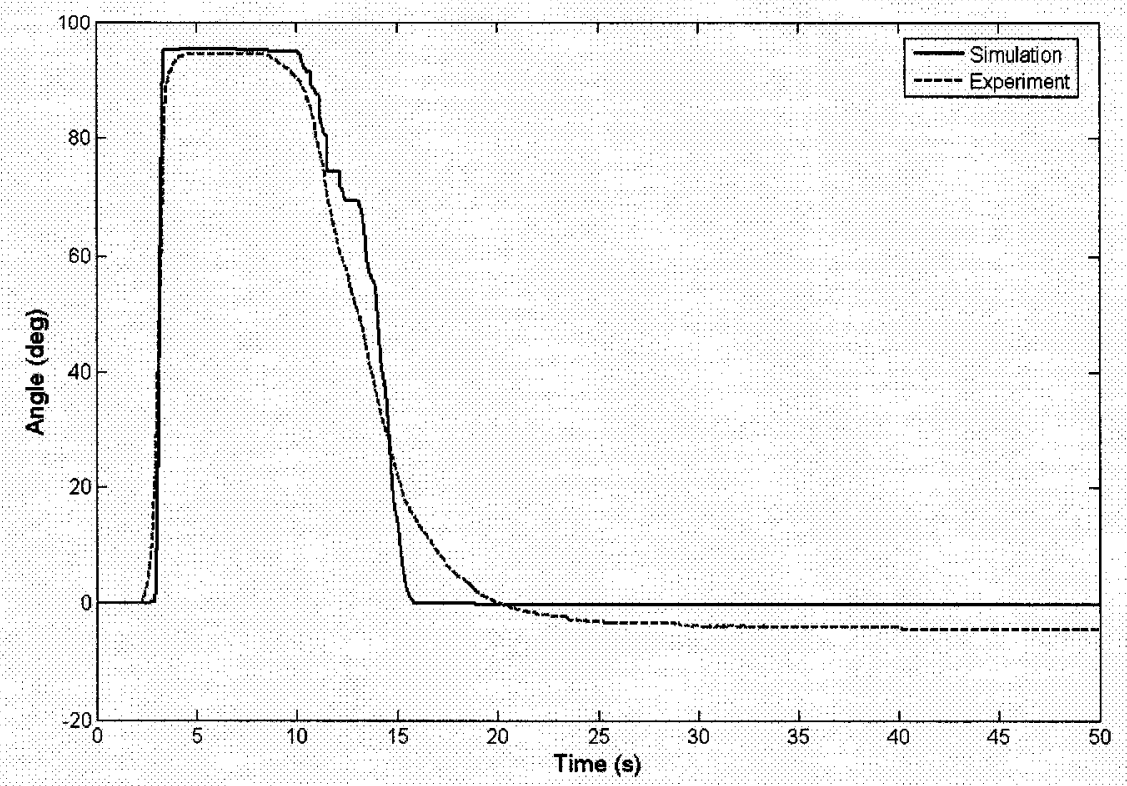

Figure 35.-Single channel model simulation results compared to experimental data.

The results of the single channel system model compare well with the results of the experiment. Although the final deflections do not match, the transformation curves correlate well. Figure 35 demonstrates the comparison between the single channel model and experimental results for an open loop actuation of the wire. The small discontinuities are attributed to noise within the temperature data.

\subsection{Dual Channel System Model}

The second model simulated the combination of two SMA wires applied against each other in antagonistic motion. No external loads were applied in this simulation, and therefore it can be said that the stress in both SMA wires is equal at all times. 


$$
\sigma_{1}=\sigma_{2}=\sigma
$$

Eq. 29

However, the system is indeterminate and therefore stress is neither known nor is constant throughout the simulation. To simplify the problem, only the top SMA channel was made active in the second model. The bottom SMA wire acted only as a constraint, much like a bias spring. The constitutive equation can be given for both SMA wires as equations 29 and 30.

$$
\begin{aligned}
& \Delta \sigma=E_{1}\left(\xi_{1}\right)\left\{\Delta \varepsilon_{1}-\varepsilon_{L} \Delta \xi_{1}+\alpha_{1}(\xi) \Delta T_{1}\right\} \\
& \Delta \sigma=E_{2}\left(\xi_{2}\right)\left\{\Delta \varepsilon_{2}-\varepsilon_{L} \Delta \xi_{2}+\alpha_{2}(\xi) \Delta T_{2}\right\}
\end{aligned}
$$

However, since the second wire is never actuated, both martensite fraction and temperature do not change. Therefore, equations 29 and 30 can be combined into equation 31, according to the constraint imposed by equation 28. Furthermore, the Young's Modulus for the second wire will be a constant assuming the second wire is entirely martensite to begin with.

$$
E_{1}\left(\xi_{1}\right)\left\{\Delta \varepsilon_{1}-\varepsilon_{L} \Delta \xi_{1}+\alpha_{1}(\xi) \Delta T_{1}\right\}=E_{M} \Delta \varepsilon_{2}
$$

Another constraint can be imposed upon the system. The change in strain in each 
wire must be equal in magnitude but opposite in direction at all times, since both wires are connected to the shaft.

$$
\Delta \varepsilon_{1}=-\Delta \varepsilon_{2}=\Delta \varepsilon_{\theta}
$$

Eq. 32

Therefore, equation 31 can be re-written as equation 33 .

$$
\Delta \varepsilon_{\theta}=\frac{E_{1}\left(\xi_{1}\right)\left[\varepsilon_{L} \Delta \xi_{1}-\alpha_{1}\left(\xi_{1}\right) \Delta T_{1}\right]}{E_{1}\left(\xi_{1}\right)+E_{M}}
$$

Furthermore, equation 30 can be re-written as equation 34.

$$
\sigma=-E_{M} \Delta \varepsilon_{\theta}+\sigma_{0}
$$

The overall system involves essentially six unknowns, $\sigma_{1}, \sigma_{2}, \varepsilon_{l}, \varepsilon_{2}, \xi_{l}$, and $\xi_{2}$. The constraints imposed by equations 28 and 32 reduce the system to three unknowns, $\sigma$, $\Delta \varepsilon_{\phi}$, and $\xi_{l}$. These three unknowns can be solved using equations 33 and 34 , as well as the conditional use of the phase transformation equations 18 and 22. The temperature time history is assumed to be given in the analysis.

Unfortunately, the phase transformation equations are non-linear. Therefore, the three equations cannot be solved using Cramer's rule. Instead, a numerical procedure based on the Newton-Raphson method is used to find a solution. 
An iterative process is used to find values for $\alpha=\sigma, \beta=\Delta \varepsilon_{\theta}$, and $\gamma=\xi_{1}$ that satisfy equations 33 and 34 and the phase transformation equation. Therefore, the following functions are created.

$$
\begin{aligned}
& F(\alpha, \beta, \gamma)=\sigma(\beta)-\alpha=0 \\
& G(\alpha, \beta, \gamma)=\Delta \varepsilon_{\theta}(\gamma)-\beta=0 \\
& H(\alpha, \beta, \gamma)=\xi_{1}(\alpha)-\gamma=0
\end{aligned}
$$

Eq. 35

An initial guess is made for $\alpha_{0}, \beta_{0}$, and $\gamma_{0}$, and input into equation 35 . The validity of the guess is then checked using equations 36 and 37 where $\eta$ is defined as the residual. This is the convergence criterion of the solution based on the relative error.

$$
\left|\frac{F\left(\alpha_{0}, \beta_{0}, \gamma_{0}\right)}{\alpha_{0}}\right|+\left|\frac{G\left(\alpha_{0}, \beta_{0}, \gamma_{0}\right)}{\beta_{0}}\right|+\left|\frac{H\left(\alpha_{0}, \beta_{0}, \gamma_{0}\right)}{\gamma_{0}}\right|=\eta
$$

$$
\eta<0.0001
$$

Eq. 37

If equations 36 and 37 are not satisfied, the Newton-Raphson algorithm is implemented using equations 38 to 41 . Matrix algebra can be used to find a solution for the three linearized equations 39 to 41 , in three unknowns, $\Delta \alpha_{1}, \Delta \beta_{1}$ and $\Delta \gamma_{1}$. Equation 38 then yields updated values for $\alpha, \beta$, and $\gamma$. 


$$
\begin{aligned}
& \alpha_{1}=\alpha_{0}+\Delta \alpha_{1} \\
& \beta_{1}=\beta_{0}+\Delta \beta_{1} \\
& \gamma_{1}=\gamma_{0}+\Delta \gamma_{1}
\end{aligned}
$$

Eq. 38

$$
F\left(\alpha_{1}, \beta_{1}, \gamma_{1}\right)=F\left(\alpha_{0}, \beta_{0}, \gamma_{0}\right)+\frac{\partial F}{\partial \alpha} \Delta \alpha_{1}+\frac{\partial F}{\partial \beta} \Delta \beta_{1}+\frac{\partial F}{\partial \gamma} \Delta \gamma_{1}
$$

Eq. 39

$$
\begin{gathered}
G\left(\alpha_{1}, \beta_{1}, \gamma_{1}\right)=G\left(\alpha_{0}, \beta_{0}, \gamma_{0}\right)+\frac{\partial G}{\partial \alpha} \Delta \alpha_{1}+\frac{\partial G}{\partial \beta} \Delta \beta_{1}+\frac{\partial G}{\partial \gamma} \Delta \gamma_{1} \\
F\left(\alpha_{1}, \beta_{1}, \gamma_{1}\right)=H\left(\alpha_{0}, \beta_{0}, \gamma_{0}\right)+\frac{\partial H}{\partial \alpha} \Delta \alpha_{1}+\frac{\partial H}{\partial \beta} \Delta \beta_{1}+\frac{\partial H}{\partial \gamma} \Delta \gamma_{1}
\end{gathered}
$$

After solving equations 39 to 41 , the new solution of equation 38 is then checked for validity. This process repeats until proper values for $\alpha, \beta$ and $\gamma$ are found.

The functions, $F(\alpha, \beta, \gamma), G(\alpha, \beta, \gamma)$ and $H(\alpha, \beta, \gamma)$ can be re-written as $F(\alpha, \beta)$, $G(\beta, \gamma)$, and $H(\alpha, \gamma)$ since each function is one-dimensional. The partial derivatives in equations 39 to 41 then reduce significantly to those in equation 42 . 


$$
\begin{array}{ll}
\frac{\partial F}{\partial \alpha}=-1, & \frac{\partial F}{\partial \beta} \neq 0, \quad \frac{\partial F}{\partial \gamma}=0 \\
\frac{\partial G}{\partial \alpha}=0, & \frac{\partial G}{\partial \beta}=-1, \quad \frac{\partial G}{\partial \gamma} \neq 0 \\
\frac{\partial H}{\partial \alpha} \neq 0, & \frac{\partial H}{\partial \beta}=0, \quad \frac{\partial H}{\partial \gamma}=-1
\end{array}
$$

Eq. 42

Furthermore, the non-zero derivatives can be expressed as in equations 43 to 45 .

$$
\frac{\partial F}{\partial \beta}=-E_{M}
$$

Eq. 43

$$
\begin{aligned}
& \frac{\partial G}{\partial \gamma}=\frac{g_{1} \gamma^{2}+g_{2} \gamma+g_{3}+g_{4}}{g_{5} \gamma^{2}+g_{6} \gamma+g_{7}} \\
& g_{1}=\left(E_{M}-E_{A}\right)^{2}\left[\varepsilon_{L}-\left(\alpha_{M}-\alpha_{A}\right) \Delta T_{1}\right] \\
& g_{2}=\left(E_{M}^{2}-E_{A}^{2}\right)\left[\varepsilon_{L}-\left(\alpha_{M}-\alpha_{A}\right) \Delta T_{1}\right] \\
& g_{3}=E_{M}\left(E_{A}-E_{M}\right)\left(\varepsilon_{L} \xi_{1 o}+\alpha_{A} \Delta T_{1}\right) \\
& g_{4}=E_{A}\left(E_{A}+E_{M}\right)\left[\varepsilon_{L}-\left(\alpha_{M}-\alpha_{A}\right) \Delta T_{1}\right] \\
& g_{5}=\left(E_{M}-E_{A}\right)^{2} \\
& g_{6}=2\left(E_{M}^{2}-E_{A}^{2}\right) \\
& g_{7}=\left(E_{M}+E_{A}\right)^{2}
\end{aligned}
$$

Eq. 44 


$$
\begin{aligned}
& \frac{\partial H}{\partial \alpha}=\left(\frac{\xi_{0} a_{A}}{2 C_{A}}\right) \sin \left[a_{A}\left(T-A_{s}-\frac{\alpha}{C_{A}}\right)\right], \quad A_{s}^{\prime}<T<A_{f}^{\prime} \\
& \frac{\partial H}{\partial \alpha}=\left(\frac{a_{M}\left(1-\xi_{0}\right)}{2 C_{M}}\right) \sin \left[a_{M}\left(T-M_{f}-\frac{\alpha}{C_{M}}\right)\right], \quad M_{f}^{\prime}>T>M_{s}^{\prime}
\end{aligned}
$$

Eq. 45

Therefore, the following matrix equation, equation 46, yields the solution to equations 39 to 41 .

$$
\left[\begin{array}{ccc}
1 & -\frac{\partial F}{\partial \beta} & 0 \\
0 & 1 & -\frac{\partial G}{\partial \gamma} \\
-\frac{\partial H}{\partial \alpha} & 0 & 1
\end{array}\right]\left[\begin{array}{l}
\Delta \alpha \\
\Delta \beta \\
\Delta \gamma
\end{array}\right]=\left[\begin{array}{l}
F\left(\alpha_{0}, \beta_{0}\right) \\
G\left(\beta_{0}, \gamma_{0}\right) \\
H\left(\alpha_{0}, \gamma_{0}\right)
\end{array}\right]
$$

Eq. 46

The model was then simulated in Matlab using a code written with the following algorithm. Figure 36 illustrates the solution algorithm as a flow chart.

1. Define material properties, transformation temperatures, maximum residual strain, Young's moduli, thermal expansion coefficients, stress influence coefficients, initial preload, and initial martensite fraction.

2. Calculate initial strain based upon initial stress.

3. Define temperature-time history (given by user input or from experimental data). This is the input to the simulation. 


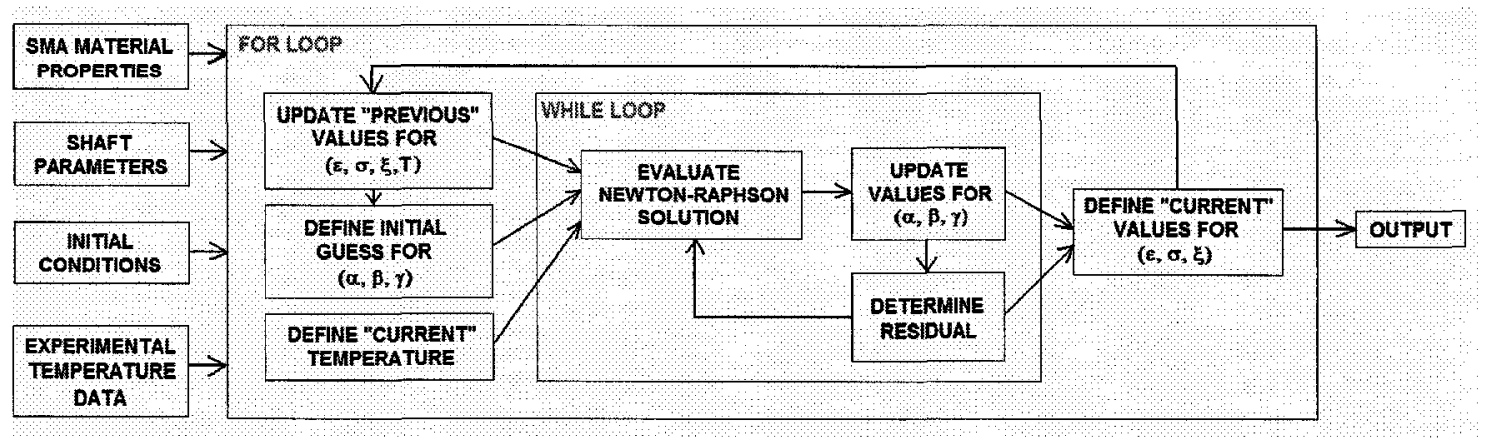

Figure 36.-Flow chart of Newton-Raphson solution method for dual channel model.

4. Begin 'for' loop (number of iterations equal to number of data points in temperature-time history). For each iteration:

(i) Define 'previous stress' equal to current stress. Define 'previous temperature' and 'next temperature' according to temperature-time history.

(ii) Define initial guess $\left(\alpha_{0}, \beta_{0}, \gamma_{0}\right)$ for Newton-Raphson method. Evaluate functions, $F\left(\alpha_{0}, \beta_{0}\right), G\left(\beta_{0}, \gamma_{0}\right)$, and $H\left(\alpha_{0}, \gamma_{0}\right)$, and residual, $\eta$.

(iii) Begin 'while' loop, under the condition that the residual is greater than the defined error bandwidth.

(iv) Solve equation 46 to determine increments in equation 38.

(v) Evaluate functions, $F\left(\alpha_{1}, \beta_{1}\right), G\left(\beta_{1}, \gamma_{1}\right)$, and $H\left(\alpha_{1}, \gamma_{1}\right)$, and residual, $\eta$.

(vi) Redefine $\left(\alpha_{0}, \beta_{0}, \gamma_{0}\right)=\left(\alpha_{\imath}, \beta_{l}, \gamma_{l}\right)$ and repeat 'while' loop if the residual is greater than the error bandwidth.

(vii) After satisfactory values for $(\alpha, \beta, \gamma)$ found set 'next stress' $=\alpha_{0}$, 'change in strain' $=\beta_{0}$, and 'next martensite fraction' $=\gamma_{0}$.

5. Repeat next 'for' loop iteration. 
6. Plot response after 'for' loop completes.

\subsection{Dual Channel Simulation Results}

The dual channel configuration model was simulated using the properties listed in table 18. As mentioned previously, only one wire was actuated during simulation of the dual channel configuration for simplicity. Temperature data from experimental open loop responses was interpolated cubically to increase the resolution of the data.

\begin{tabular}{|cc|}
\hline Property & Value \\
\hline Shaft Radius, $\mathrm{r}$ & $6.35 \mathrm{~mm}$ \\
SMA Wire Length, $\mathrm{L}$ & $270 \mathrm{~mm}$ \\
SMA Wire Diameter & $0.381 \mathrm{~mm}$ \\
Preload & $2.1 \mathrm{MPa}$ \\
\hline
\end{tabular}

Table 18.-Dual channel model simulation parameters.

Similar to the single channel model results, the dual channel model shows good correlation over the transformation curves and some deviation of the maximum deflections. Figure 37 demonstrates the comparison between the dual channel model simulation and experimental results for an open loop actuation of a single wire. 


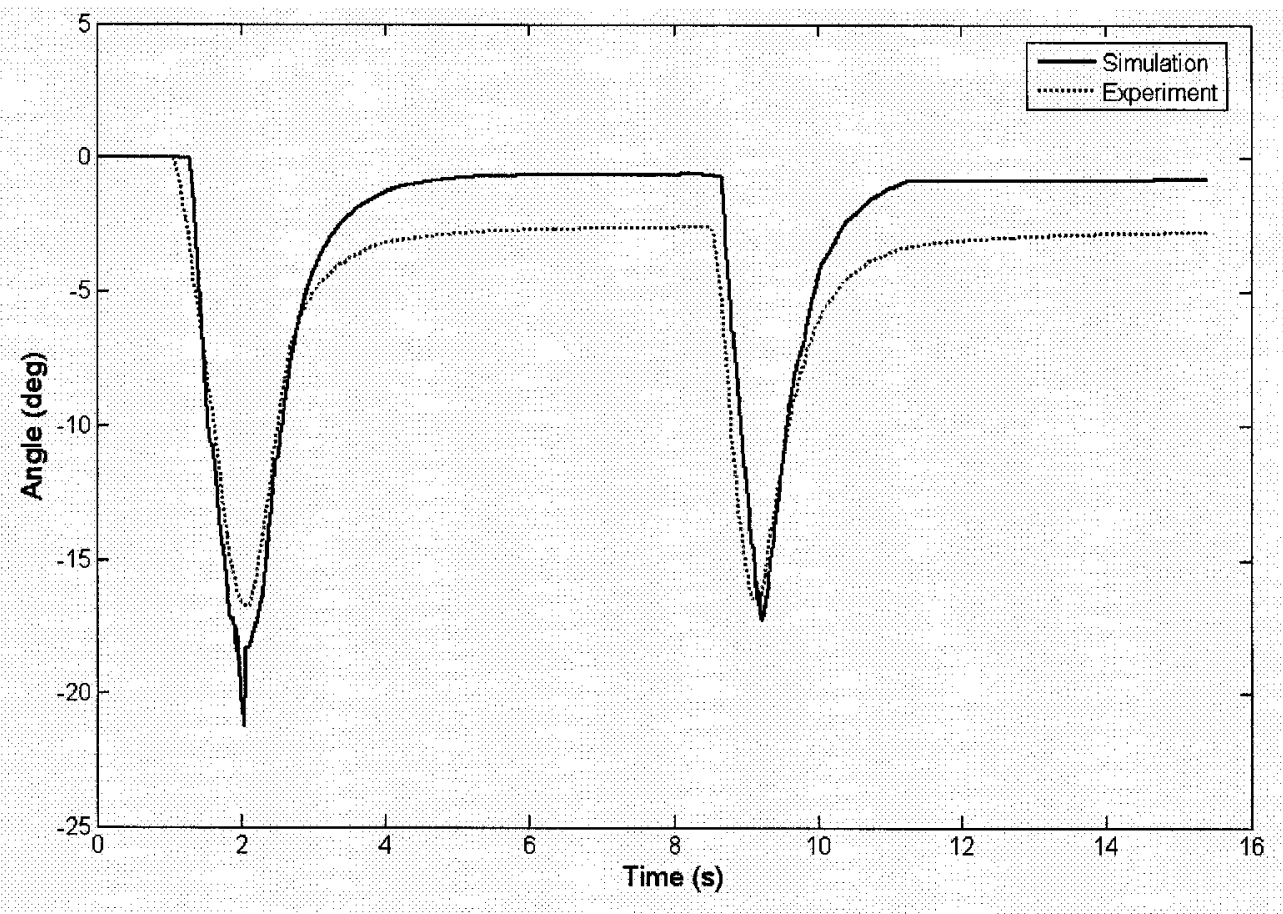

Figure 37.-Dual channel model simulation results compared to experimental data. 


\section{Applications to SHARCS Project}

The use of SMA wires for actuating the actively controlled tip was investigated for the SHARCS project. Sufficient moment must be provided by the SMA actuator system to overcome the thrust distribution along the tip, as well as the centrifugal load during deflection. The Blade Element Momentum Theory (BEMT) model was used to simulate the SHARCS blade aerodynamics [23]. This model was then combined with a model of the centrifugal loading on the deflected tip to determine the actuator requirements and investigate the feasibility of an SMA actuator for the SHARCS blade.

\subsection{SHARCS Blade Geometry}

The SHARCS blade is a scaled helicopter rotor blade with a tip radius of $1.096 \mathrm{~m}$. Carbon and glass fibre composite layers form the symmetric NACA0015 airfoil which also acts as the blade structure to maximize interior space. Figure 38 shows the SHARCS

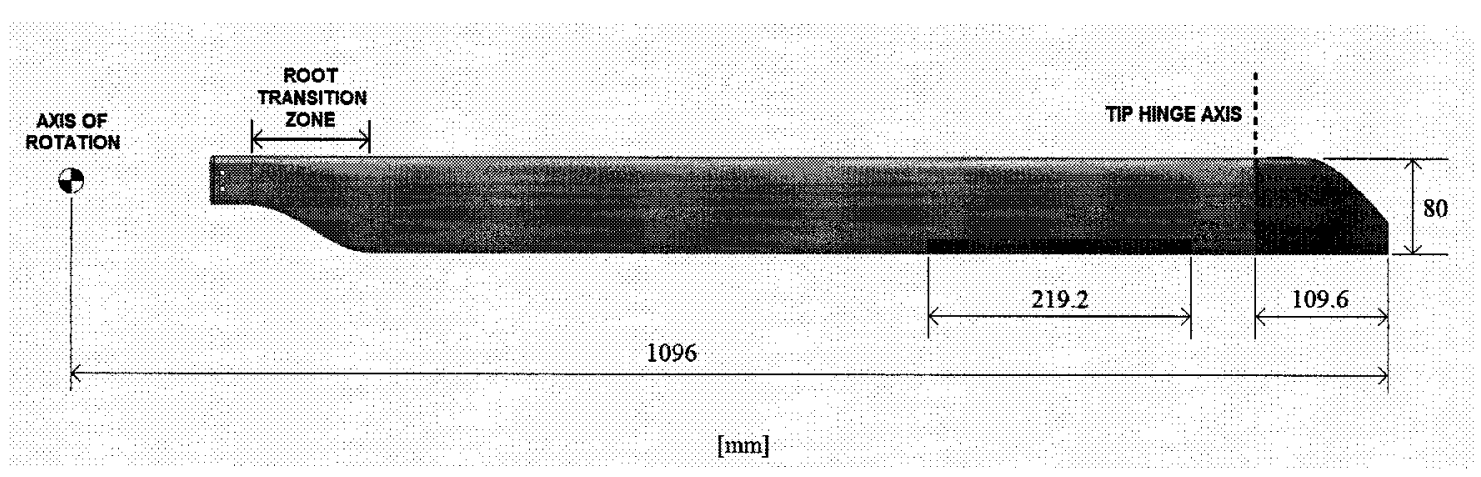

Figure 38.-SHARCS blade top view. 


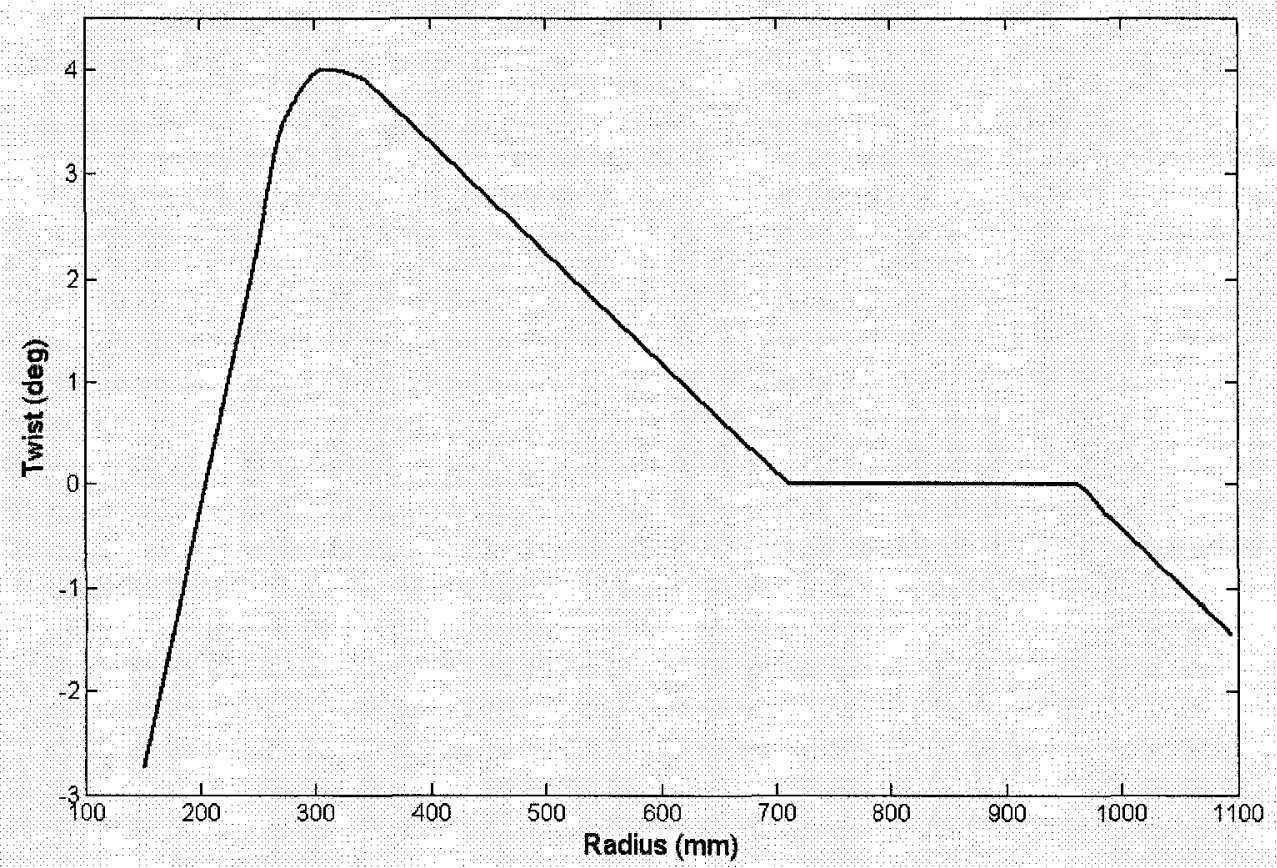

Figure 39.-SHARCS geometric twist distribution.

blade with the actively controlled flap and tip shaded and some main dimensions. Geometric twist distribution in the spanwise direction is introduced for aerodynamic purposes and is shown in figure 39.

The numerical analysis of the aerodynamic loading required the discretization of the blade into two-dimensional elements in the spanwise direction. Each element was assumed to have a constant value of chord, twist, and aerodynamic load. The BEMT model employed 1000 elements from the start of the root transition zone to the tip. Aerodynamic performance of the root transition zone was unknown therefore it was conservatively assumed to have the same properties as the main blade section, producing additional loading. The centrifugal loading model used 100 elements from the hinge located at $90 \%$ radius to the tip. 


\subsection{Operating Conditions}

Aerodynamic loads were determined for the SHARCS blade operating in the hover flight regime at sea level with air density $\rho=1.225 \mathrm{~kg} / \mathrm{m}^{3}$. Similarity requirements for the SHARCS blade determine the rotational velocity of the system, which is approximately 1550 RPM. Superimposed upon the blade geometric twist is the collective pitch setting summed uniformly along the blade. Collective pitch settings of up to approximately $12^{\circ}$ were investigated. The actively controlled tip is required to achieve anhedral angles of up to $20^{\circ}$, although various settings were investigated.

\subsection{Blade Tip Loading Model}

The aerodynamic loading of the rotor blade and expected hinge moment at the tip was predicted using Blade Element Momentum Theory (BEMT). Prandtl's tip loss factor was also used in order to more accurately model the load distribution along the blade tip. Equation 47 represents a differential element of the thrust coefficient, $C_{T}$, for a rotor blade, where $a$ is the slope of the lift coefficient as a function of angle of attack, $\theta$ is the blade twist, $\lambda$ is the inflow ratio, $r$ is the normalized radial location, and $\sigma$ is the rotor solidarity given by equation 48 .

$$
d C_{T}=\frac{\sigma a}{2}(\theta r-\lambda) r d r
$$




$$
\sigma=\frac{N c}{\pi r}
$$

Eq. 48

The variable $N$ is the number of blades and $c$ is the chord length. The inflow ratio is given as a non-uniform function shown in equation 49 , where $F$ is the Prandtl tip loss factor and is also a function of the radial location.

$$
\lambda=\frac{\sigma a}{16 F} \sqrt{1+F\left(\frac{32}{\sigma a}\right) \theta r}-1
$$

Eq. 49

The Prandtl tip loss factor is given by equation 50 and is a function of the inflow ratio.

$$
F=\frac{2}{\pi} \cos ^{-1}\left(e^{\frac{N(r-1)}{2 \lambda}}\right)
$$

Eq. 50

The distribution of the Prandtl tip loss factor is assumed to be uniform and equal to unity for initiating the iterative solution. Based upon this assumption, the inflow ratio distribution is calculated from equation 49. The inflow ratio distribution is then used to calculate a new Prandtl tip loss factor distribution. This process is repeated until the solution has converged. The thrust coefficient distribution along the blade is then determined using equation 47. 
The actual thrust is given by the relationship shown in equation 51 , where $\rho$ is the air density, $A$ is the rotor disk area, $\Omega$ is the rotational velocity, and $R$ is the blade radius.

$$
T=\rho A(\Omega R)^{2} C_{T}
$$

Differentiation leads to the relationship shown in equation 52 . The chain rule is then applied in equations 53 to 55 and the actual thrust distribution can be determined.

$$
\frac{d T}{d r}=\rho A(\Omega R)^{2} \frac{d C_{T}}{d r}
$$

$$
\frac{d T}{d x}=\frac{d T}{d r} \frac{d r}{d x}
$$

$$
d x=R d r
$$

Eq. 54

$$
\frac{d T}{d x}=\frac{1}{R} \frac{d T}{d r}
$$

The variable $x$ is the actual radial location along the blade. Figure 40 shows the thrust distribution for the SHARCS blade. The aerodynamic bending moment distribution is then determined using equation 56 , where the dummy variable $z$ is used to integrate 
from each location $x$ to the tip of the blade. Figure 41 shows the aerodynamic bending moment.

$$
M_{A}=\int_{x}^{R}(z-x) \frac{d T}{d x} d z
$$

Eq. 56

Due to the tip flap anhedral angular deflection, there will also be a bending moment generated by centrifugal forces. Assuming positive anhedral angles in the downward direction, the centrifugal bending moment at the tip flap hinge is represented by equation 57 , where $\mu$ is the blade mass per unit area, $h$ is the radial location of the tip flap hinge and $\beta$ is the anhedral angle.

$$
M_{C}=\mu \Omega^{2} \sin \beta \int_{h}^{R} c x(x-h) d x
$$

Bending moments from aerodynamic and centrifugal forces both act in the same direction. Therefore equation 58 represents the net bending moment acting on the tip flap hinge, where the superscript $h$ represents the aerodynamic bending moment evaluated at the tip flap hinge. The net hinge moment for various collective pitch and anhedral angle settings is shown in figure 42 .

$$
M_{T}=M_{A}^{h}+M_{C}
$$




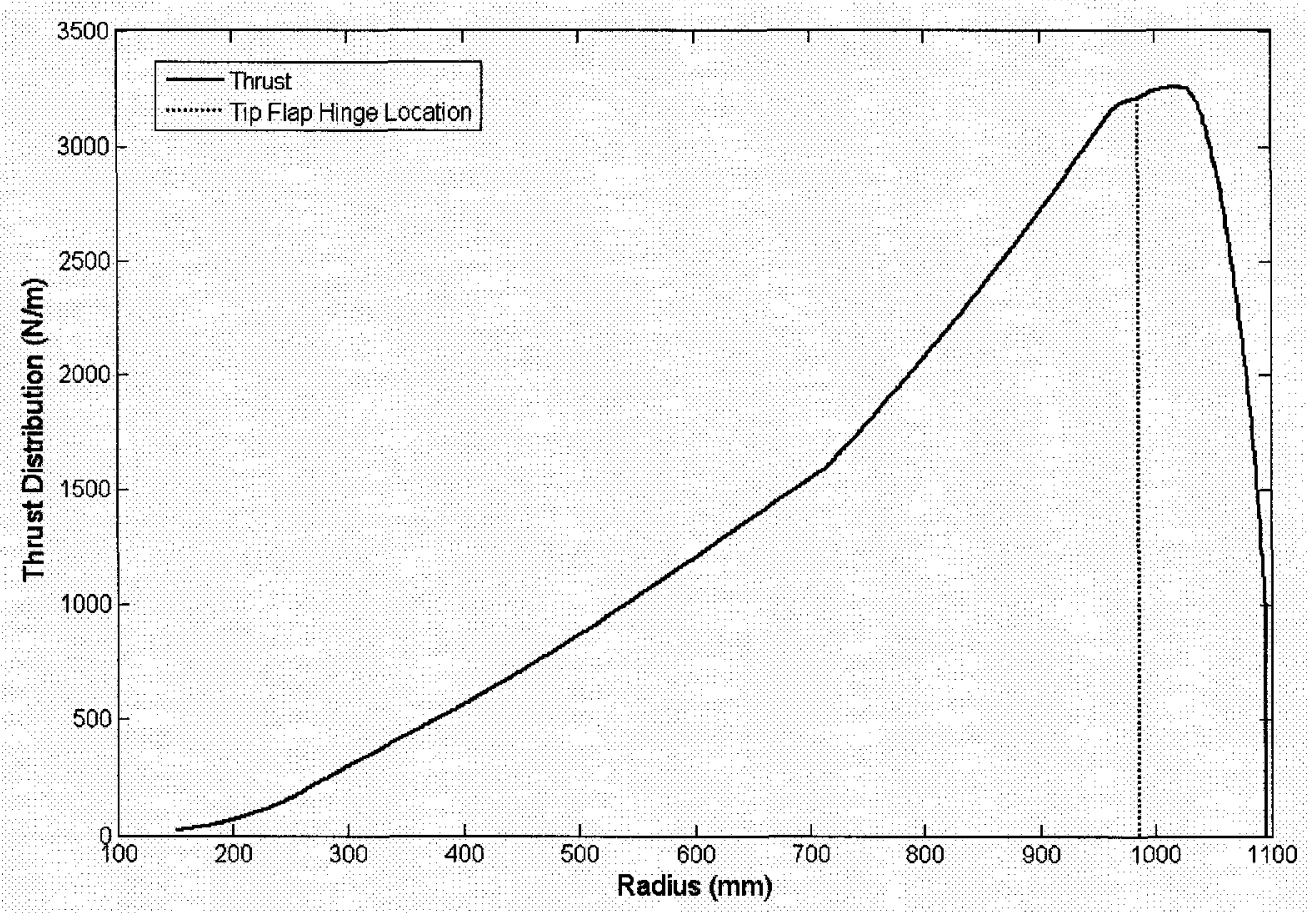

Figure 40.-SHARCS thrust distribution.

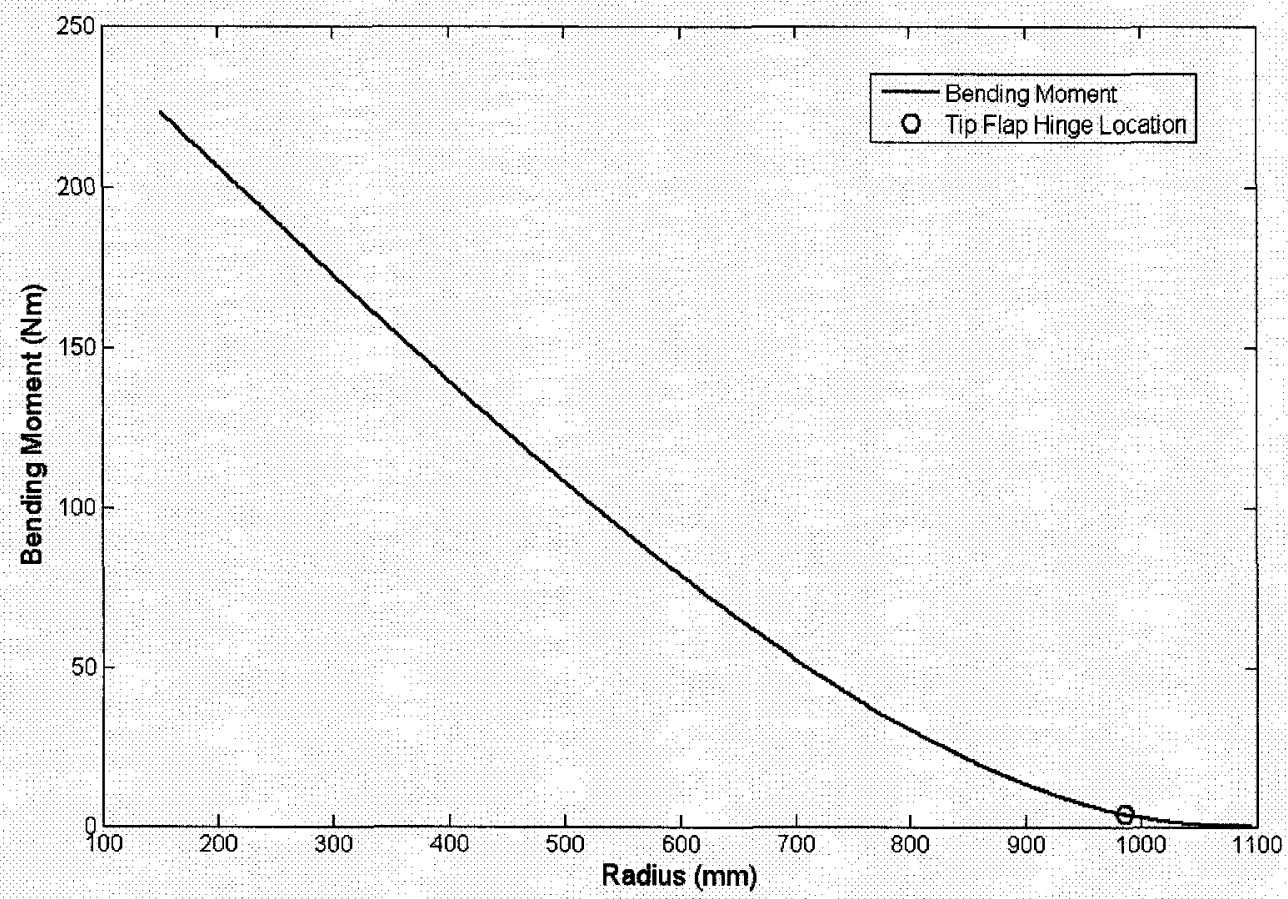

Figure 41.-SHARCS bending moment. 


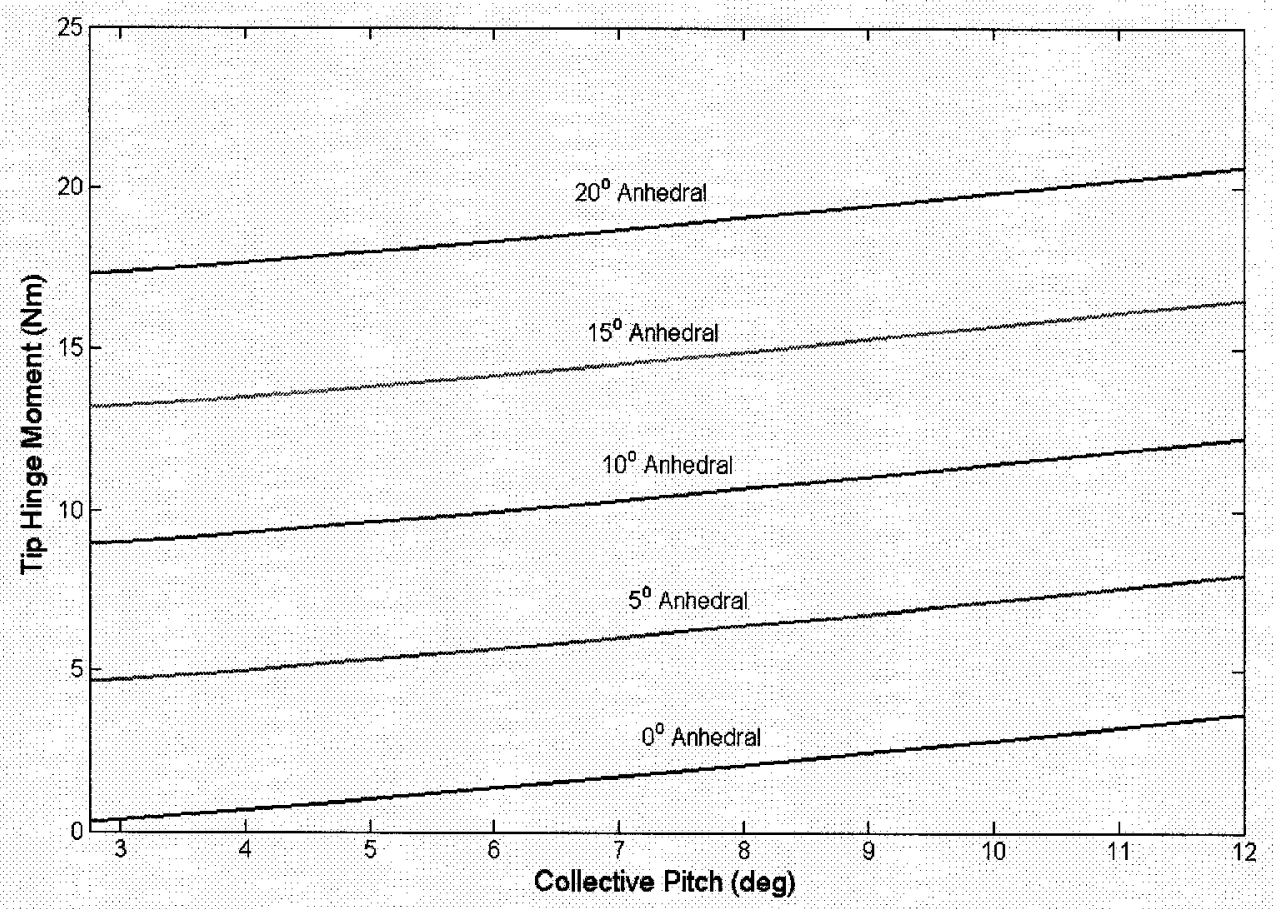

Figure 42.-SHARCS net hinge moment for various anhedral angle settings.

\subsection{SMA Tip Actuator Feasibility}

The feasibility of an SMA actuator for the actively controlled tip on the SHARCS blade was studied by simplifying the mechanism as a hinge similar to the experimental setup. It was conceived that Flexinol SMA actuator wires would be mounted to the hinge antagonistically. Each wire is capable of approximately $20 \mathrm{~N}$ force, and has a diameter of $0.381 \mathrm{~mm}$. Figure 43 demonstrates a hinge configuration possible within the SHARCS blade. Although it is desirable to have a large length to accommodate a number of wires 


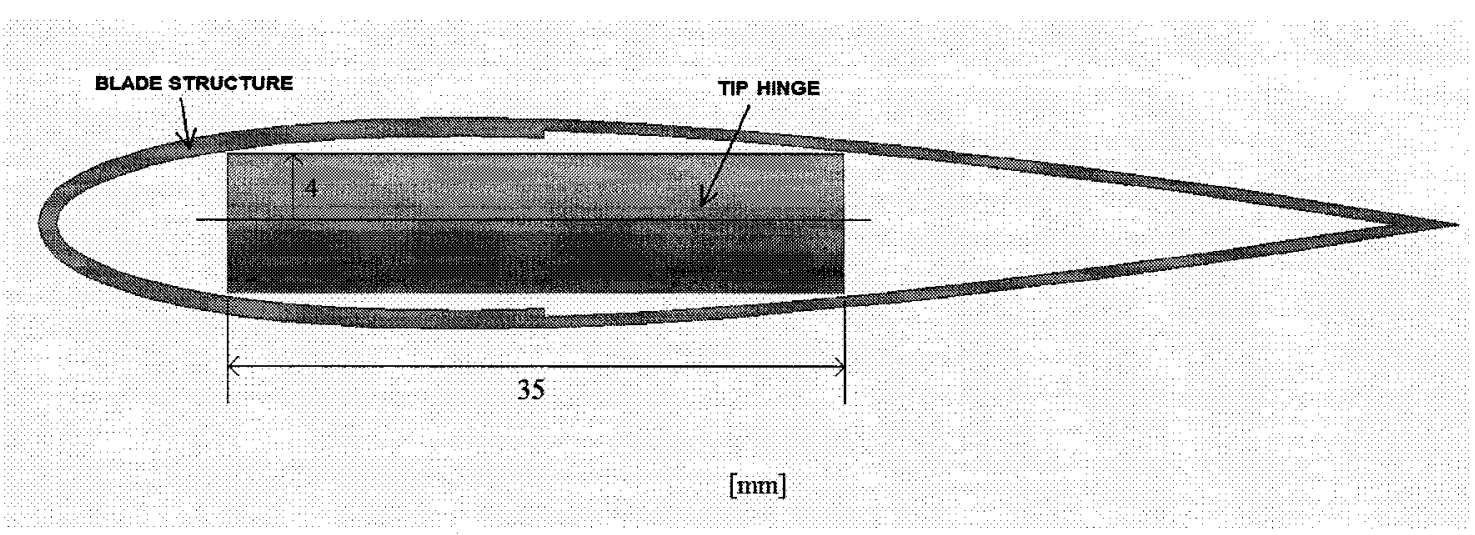

Figure 43.-Possible configuration for SHARCS tip hinge.

and a large radius to maximize applied torque, the limits of the blade structure prevent both demands from being satisfied.

Assuming a $12^{\circ}$ collective pitch setting and a $20^{\circ}$ anhedral tip deflection, the hinge moment required by the actuator can be found from figure 42 . The value of the hinge moment at these settings is approximately $20 \mathrm{Nm}$. Transforming this load into a force using a moment arm of $4 \mathrm{~mm}$ from figure 43 , the required actuator force is $5000 \mathrm{~N}$. Assuming a maximum load of $20 \mathrm{~N}$ for each Flexinol actuator wire, approximately 250 wires would be required to support the maximum expected load without any safety factor. This number of SMA wires is infeasible since the required hinge length would be approximately $95 \mathrm{~mm}$. Given a hinge length of $35 \mathrm{~mm}$ and wire diameter of $0.381 \mathrm{~mm}$, 91 wires could be installed and achieve a hinge moment of approximately $7.3 \mathrm{~N}$. However, this corresponds to an anhedral deflection of approximately $5^{\circ}$ which is unsatisfactory. 


\subsection{Full-Scale Actuator Feasibility}

The SHARCS actively controlled tip could possibly be applied to a full scale helicopter rotor blade in the future. A simple feasibility analysis was performed for the SMA actuator on the full scale SHARCS blade. Main dimensions and operating conditions of the full scale SHARCS blade, as shown in table 18, were assumed to be equal to those from a Bo-105 helicopter blade [24]. However, the airfoil remained a NACA0015 section and the normalized chord and twist distributions remained similar to those of the original SHARCS blade.

\begin{tabular}{|ccc|}
\hline Parameter & \multicolumn{2}{c|}{ Value } \\
& SHARCS & Bo-105 \\
\hline Radius & $1.096 \mathrm{~m}$ & $4.938 \mathrm{~m}$ \\
Chord & $0.080 \mathrm{~m}$ & $0.395 \mathrm{~m}$ \\
Angular Velocity & $1555 \mathrm{RPM}$ & $383.1 \mathrm{RPM}$ \\
\hline
\end{tabular}

Table 19.-Parameters assumed from a full scale Bo-105 rotor blade [23].

The analysis of the blade tip loads was repeated using these parameters. A similar number of elements were used for both the blade and the tip. The resulting loads are presented in figure 44. Although the hinge moment is greater for the full scale blade, the interior space available is larger. However, the structure of a full scale SHARCS blade has not been determined therefore the hinge geometry is difficult to predict. A simplified analysis was performed in which the hinge geometry in figure 43 was scaled by the ratio of chord lengths. Larger Flexinol actuator wire was also chosen, with diameter $0.508 \mathrm{~mm}$ and maximum tension $35 \mathrm{~N}$. Based upon these assumptions, the hinge length and radius 


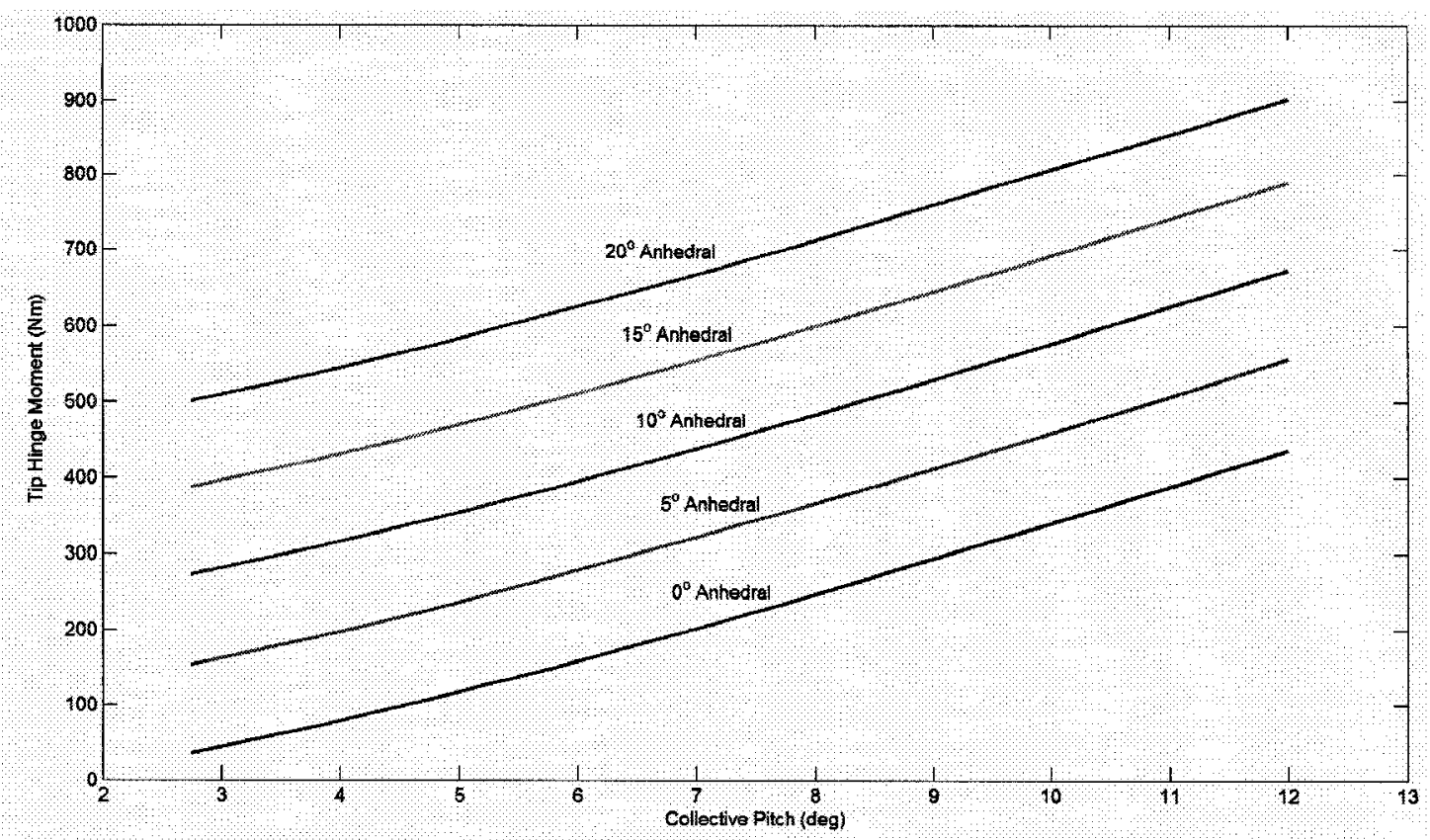

Figure 44.-Tip hinge moment for a full scale SHARCS blade based upon Bo-105 geometry.

were $172.8 \mathrm{~mm}$ and $19.75 \mathrm{~mm}$ respectively. Therefore, approximately 340 actuator wires could be accommodated, yielding a maximum actuation moment of $235.1 \mathrm{Nm}$. According to figure 44 the maximum tip hinge moment is approximately $800 \mathrm{Nm}$. This suggests a full scale antagonistic SMA wire actuator design is infeasible. However, such an analysis should be repeated in the future given more accurate parameters for a full scale SHARCS blade. 


\section{Conclusions}

Research was successfully undertaken to investigate the feasibility of shape memory alloy wires as actuators arranged antagonistically for angular position control of a shaft and model the results for comparison.

Control was achieved for a single channel actuator acting against a bias load and a dual channel set of actuators configured antagonistically. The system was able to track a target angular position with acceptable overshoot and settling time. Stability was maintained during random manual excitation of the shaft. This suggested that an actuator system based upon shape memory alloy wires could be a viable solution for control of a helicopter rotor blade tip flap.

Mathematical modeling of the system proved that the Liang \& Rogers constitutive shape memory alloy model is appropriate for comparison. Overall, the results of the simulations demonstrated a good correlation between the model and experimental data. Errors were primarily attributed to friction on the shaft and noise within the sensor data. Another simplification leading to error was that intermediate connecting parts, such as the pulley block and turnbuckles, contributed no additional strain. The model also assumes the entire actuator wire reaches a uniform temperature, although heat will be transferred to the apparatus at the boundaries of the wire.

The results of the blade tip loading model suggest that the use of shape memory alloy wires as actuators for the SHARCS blade is infeasible due to the magnitude of the net tip moment from aerodynamic and centrifugal loads. This conclusion is drawn from 
investigating the number of actuator wires required to overcome the net tip flap hinge moment. A similar system installed in a full scale SHARCS blade may be feasible if the composite structure can accommodate larger hinge geometry. 


\section{References}

[1] Calkins, Frederick T., James H. Mabe, George W. Butler. Boeing's Variable Geometry Chevron: Morphing Aerospace Structures for Jet Noise Reduction. Smart Structures and Materials 2006: Industrial and Commercial Applications of Smart Structures Technologies, Proceedings of SPIE Vol. 6171, 2006.

[2] Turner, Travis L., Ralph D. Buehrle, Roberto J. Cano and Gary A Fleming. Modeling, Fabrication, and Testing of a SMA Hybrid Composite Jet Engine Chevron Concept. Journal of Intelligent Material Systems and Structures, Vol. 17, June 2006, pages 483-497.

[3] Birch, Matthew C., Roger D. Quinn, Geon Hahm, et. al.. A Miniature Hybrid Rotor Propelled by Legs. Case Western Reserve University, Cleveland, Ohio, 2001.

[4] Reynaerts, Dominiek, Jan Peirs and Hendrik Van Brussel. Design of a Shape Memory Alloy Actuated Implantable Drug Delivery System. Department of Mechanical Engineering, Katholieke Universiteit Leuven, Heverlee, Belgium, 1996.

[5] Ikuta, Koji. Micro-Miniature Shape Memory Alloy Actuator. Department of Mathematical Engineering and Information Physics, Faculty of Engineering, University of Tokyo, Tokyo, Japan, 1990.

[6] Bütefisch, Sebastian and Stephanus Büttgenbach. New Differential-Type SMA Actuator for a Miniature Silicon Gripper. Smart Structures and Devices, Proceedings of SPIE Vol. 4235, 2001, pages 102-107. 
[7] Velázquez, Ramiro, Edwige Pissaloux, Jerome Szewczyk and Moustapha Hafez. Miniature Shape Memory Alloy Actuator for Tactile Binary Information Display. Proceedings of the 2005 IEEE International Conference on Robotics and Automation, Barcelona, Spain, April 2005.

[8] Willey, Cliff E.. Two SMA-Actuated Miniature Mechanisms. Johns Hopkins University Applied Physics Laboratory \& NASA Goddard Space Flight Center, http://www.nasatech.com/Briefs/May05/GSC14705-1.html, 2005.

[9] Yoshida, Eiichi, Satoshi Murata, Shigeru Kokaji, Kohji Tomita and Haruhisa Kurokawa. A Micro-Size Modular Machine Using Shape Memory Alloy (SMA). Mechanical Engineering Laboratory, AIST, MITI, Ibaraki, Japan, 2003.

[10] Straub, Friedrich K., Dennis K. Kennedy, David B. Domzalski, Ahmed A. Hassan, Hieu Ngo, V. Anand and Terry Birchette. Smart Material Actuated Rotor Technology (SMART). Journal of Intelligent Material Systems and Structures, Vol. 15, April 2004, pages 249-260.

[11] Serafini, Jacopo, Claudio Testa, Stefania Leone and Massimo Gennaretti. Numerical Investigation on the Aeromechanical Behaviour in Forward Flight of a Helicopter Blade with Integrated Smart Morphing Actuator. Mechanical \& Industrial Eng. Dept., University Roma Tre, Italy, 2007.

[12] Lynch, B., F. Nitzsche, D. Feszty, M. Cha, D. Gransden, K. Khomutov, A. Mander, G. Oxley, and F. D. Ulker. Development of a Smart Rotorcraft Blade for Noise and Vibration Attenuation. Proceedings of CASI Aero Conference, Toronto, Canada, April, 2007.

[13] Nitzsche, F., D. Zimcik, V. Wickramasinghe, and C. Yong. Control Laws for an Active Tunable Vibration Absorber Designed for Aeroelastic Damping Augmentation. The Aeronautical Journal, Vol. 108, No. 1079, 2004, pages 35-42. 
[14] Nitzsche, F. and G. Oxley. Smart Spring Control of Vibration and Noise in Helicopter Blades. AIAA Paper 2005-2270, presented at the 46th AIAA/ASME/ASCE/AHS/ASC Structures, Structural Dynamics and Materials Conference, Austin, Texas, April, 2005.

[15] Kloeppel, V. and B. Enenkl. Rotor Blade Control by Active Helicopter Servo Flaps. International Forum on Aeroeleasticty and Structural Dynamics (IFADS), Munich, Germany, Paper No. IF-158, July 2005.

[16] Davis, G.L., D. Feszty, F. Nitzsche. Trailing Edge Flow Control for the Mitigation of Dynamic Stall Effects. Paper No. 053, 32nd European Rotorcraft Forum, Florence, Italy, Sep 2005.

[17] Feszty D., E.A. Gillies, M. Vezza. Alleviation of Airfoil Dynamic Stall Moments via Trailing Edge Flap Flow Control. American Institute of Aeronautics and Astronautics Journal, Vol. 42, No.1, January 2004, pages 17-25.

[18] Nitzsche, F., D. Feszty, D. Waechter, E. Bianchi, S. Voutsinas, M. Gennaretti, G. Coppotelli, G.L. Ghiringhelli. The SHARCS Project: Smart Hybrid Active Rotor Control System for Noise and Vibration Attenuation of Helicopter Rotor Blades. Paper No. 052, 31st European Rotorcraft Forum, Florence, Italy, September 2005.

[19] Friedmann, P.P.. Vibration and Noise Reduction Using Actively Controlled Flaps-Their Evolution and Potential for Improving Rotorcraft Technology. Paper No. ISF-079, IFASD Conference, Munich, Germany, 2005.

[20] Dynalloy, Inc. http://www.dynalloy.com/index.html, August 2007. 
[21] Zak, A.J., M.P. Cartmell, W.M. Ostachowicz and M. Wiercigroch. Onedimensional Shape Memory Alloy Models for use with Reinforced Composite Structures. Smart Materials and Structures, Vol. 12, 2003, pages 338-346.

[22] Liang, C. and C.A. Rogers. A Multi-dimensional Constitutive Model for Shape Memory Alloys. Journal of Engineering Mathematics, Vol. 26, 1992, pages 429443.

[23] Johnson, Wayne. Helicopter Theory. Dover Publications, Inc., Mineola, N.Y., 1980.

[24] Munksy, Brian, Farhan Gandhi and Lionel Tauszig. An Analysis of Helicopter Blade-Vortex Interaction Noise with Flight Path or Attitude Modification. Presented at the American Helicopter Society $58^{\text {th }}$ Annual Forum, Montreal, Canada, June 11-13, 2002. 